\title{
A Critical Review of Global Curriculum Development, Content and Implementation in Oncology
}

Citation for published version (APA):

Giuliani, M. E. (2020). A Critical Review of Global Curriculum Development, Content and Implementation in Oncology. [Doctoral Thesis, Maastricht University]. Datawyse / Universitaire Pers Maastricht. https://doi.org/10.26481/dis.20200519mg

Document status and date:

Published: 01/01/2020

DOI:

10.26481/dis.20200519mg

Document Version:

Publisher's PDF, also known as Version of record

\section{Please check the document version of this publication:}

- A submitted manuscript is the version of the article upon submission and before peer-review. There can be important differences between the submitted version and the official published version of record.

People interested in the research are advised to contact the author for the final version of the publication, or visit the DOI to the publisher's website.

- The final author version and the galley proof are versions of the publication after peer review.

- The final published version features the final layout of the paper including the volume, issue and page numbers.

Link to publication

\footnotetext{
General rights rights.

- You may freely distribute the URL identifying the publication in the public portal. please follow below link for the End User Agreement:

www.umlib.nl/taverne-license

Take down policy

If you believe that this document breaches copyright please contact us at:

repository@maastrichtuniversity.nl

providing details and we will investigate your claim.
}

Copyright and moral rights for the publications made accessible in the public portal are retained by the authors and/or other copyright owners and it is a condition of accessing publications that users recognise and abide by the legal requirements associated with these

- Users may download and print one copy of any publication from the public portal for the purpose of private study or research.

- You may not further distribute the material or use it for any profit-making activity or commercial gain

If the publication is distributed under the terms of Article $25 \mathrm{fa}$ of the Dutch Copyright Act, indicated by the "Taverne" license above, 


\section{A Critical Review of Global Curriculum Development, Content and Implementation in Oncology}

Meredith Elana Giuliani 
The research reported here has been carried out at Mastricht University

In the School of Health Professions Education

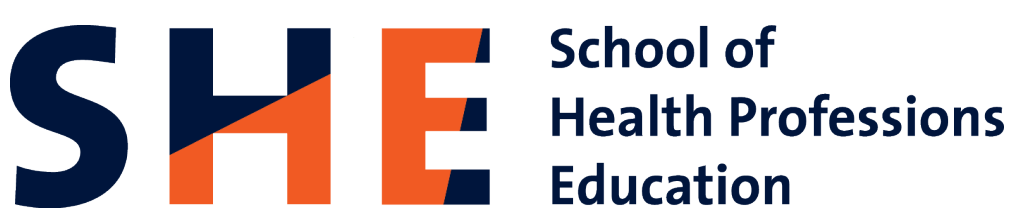

This work was supported in part by a Mapping the Landscape, Journeying Together grant from the Arnold P. Gold Foundation

(C) copyright

Printing: Datawyse | Universitaire Pers Maastricht ISBN:

\section{Cover Art Acknowledgement:}

Gail Giuliani. Calm, 2015. acrylic, $48 \times 36$ inches 


\section{A Critical Review of Global Curriculum Development, Content and Implementation in Oncology}

\section{Dissertation}

to obtain the degree of Doctor at Maastricht University, on the authority of the Rector Magnificus, Prof. dr. Rianne M. Letschert

in accordance with the decision of the Board of Deans, to be defended in public on Tuesday, 19 May 2020 at 14.00 hours

by

Meredith Elana Giuliani 


\section{Promotor:}

Prof. Dr. Erik W Driessen

\section{Co-promotors:}

Dr. Janneke M Frambach

Dr. Maria Athina (Tina) Martimianakis, University of Toronto, Toronto

\section{Assessment Committee:}

Prof. Dr. Anja Krumeich (Chair)

Prof. Dr. Liesbeth Boersma

Prof. Dr. Ming Jung Ho, Georgetown University, Georgetown

Dr. Mariëlle Jippes, Erasmus Medical Centre, Rotterdam

Dr. Dominique Waterval 
"The more one is able to leave one's cultural home, the more easily is one able to judge it, and the whole world as well, with the spiritual detachment and generosity necessary for true vision. The more easily, too, does one assess oneself and alien cultures with the same combination of intimacy and distance."

Edward W. Said, Orientalism 



\section{Table of Contents}

Chapter 1 Introduction to Neocolonialism and the Advent of Global Curricula in Oncology

Chapter 2 Motivation and Challenges in the Development of Global Medical Curricula: A scoping review

Accepted for publication: Giuliani M, Martimianakis M, Broadhurst M, Papadakos J, Fazelad R, Driessen E, Frambach J. Motivation and Challenges in the Development of Global Medical Curricula: A scoping review. Accepted to Academic Medicine December 2019.

Chapter 3 A Critical Review of Representation in the Development of Global Oncology Curricula and the Influence of Neocolonialism

Published as: Giuliani M, Frambach J, Broadhurst M, Papadakos J, Fazelad R, Driessen

E, Martimianakis M. A Critical Review of Representation in the Development of Global Oncology Curricula and the Influence of Neocolonialism. BMC Med Educ. $2020 \mathrm{Mar}$ 30;20(1):93.

Chapter 4 Humanism in Global Oncology Curricula: An Emerging Priority

Published as: Giuliani M, Martimianakis MA, Broadhurst M, Papadakos J, Fazelad R, Driessen E, Frambach J. Humanism in global oncology curricula: an emerging priority. Curr Oncol. 2020

Chapter 5 Exploring Glocalization in the Construction and Implementation of Global Curricula

Published as: Giuliani M, Frambach J, Driessen E, Martimianakis M. Exploring Glocalization in the Construction and Implementation of Global Curricula.Journal of Cancer Education. 2020

Chapter 6 Exploring Implementation of the ESTRO Core Curriculum at the National Level

Published as: Giuliani M, Athina Martimianakis M, Benstead

K, Grau Eriksen J, Verfaillie C, Van Egten V, Umakanthan B, Driessen E, Frambach

J. Exploring Implementation of the ESTRO Core Curriculum at the National Level. Radiother Oncol. 2020

Chapter 7 Discussion

Summary

Samenvatting

Valorisation

Acknowledgements

About the author 


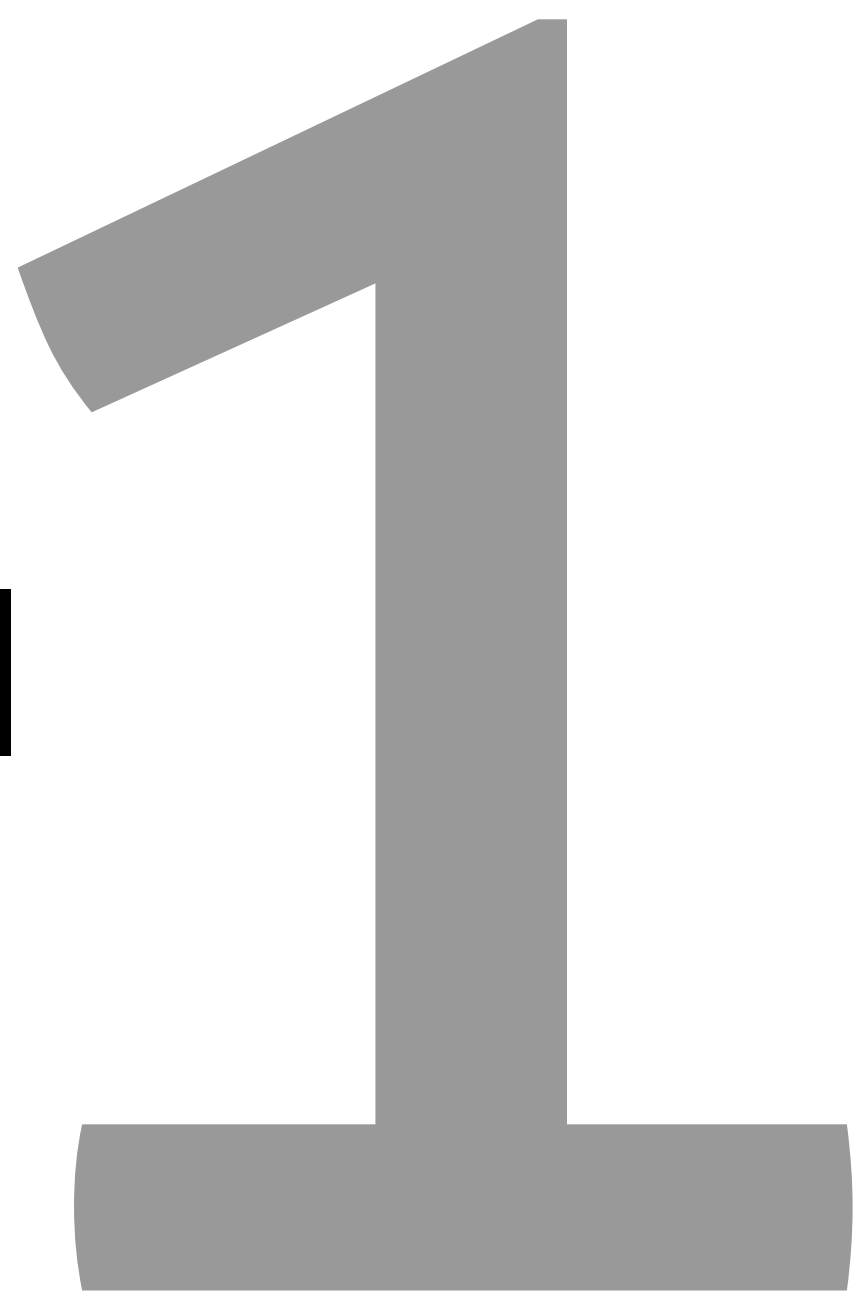




\section{Introduction to Neocolonialism and the Advent of Global Curricula in Oncology}

\section{Footnote:}

For the purposes of the introduction and discussion chapters the term 'I' will be used to denote the nature of being a student submitting a dissertation. For the purposes of the other chapters in this dissertation the terms 'we' and 'our' will be used to reflect the collaborative development and writing approaches used in the conduct, analysis and publication of the studies. 
This chapter will serve as an introduction to the core concepts which form the foundation of this dissertation. In addition, it will present the background and rationale for conducting this work, the aims and research questions which will be addressed, and the methodology employed to accomplish these aims. Finally, this chapter will include reflexivity statements on my position and background and how this has influenced the development and conduct of the studies.

The understanding of the influence of neocolonialism on medical education is growing ${ }^{1}$. Neocolonialism refers to the phenomenon of imperialism which is associated with global capitalism and the activities of Western media ${ }^{2}$. As there are growing calls for curricular reform and standardization in medical education ${ }^{3}$, the impact of neocolonialism and influences such as cultural hegemony ${ }^{4}$ in the development of curricula in medical education deserve further study. Oncology provides a rich environment to study neocolonialism because global curricula have been generated in this field and have been implemented with mixed success. One may postulate that perhaps the imposition of Western values globally may perpetuate neocolonial relationships, which may drive a mismatch between global priorities and local needs ${ }^{5}$. Given the growing health human resource crisis in oncology, driven by the need to meet the rapid rise in the incidence of cancer, there are urgent calls for novel curricula and mass training of oncology professionals. Using a critical stance, inspired by anti-colonial approaches, this dissertation will identify and describe the perceived premises for global curricula. By using oncology as a case example, this work will also explore unintended aspects of pursuing global curricula without actively considering the historical socio-political relationships that have perpetuated differences in health professional education around the world.

\section{Globalization and Healthcare}

Globalization is a term connoting an openness to trade, ideas, investment, people and culture that transcends national borders ${ }^{6,7}$. It denotes influence on an international scale and the growth of globalization is driven by advances in transportation and communication technologies. Globalization is also linked to a perceived greater interdependence between countries $^{8}$. While globalization is not a new phenomenon, the concept of 'neoliberal globalization' dates back to the late twentieth century and the subsequent studies and discussions in this dissertation will focus primarily on trends in globalization in the past three decades 9 . A trend within globalization is the phenomenon of Westernization. This is a particular form of universalization, which is linked to neocolonialism ${ }^{8}$, and is the promotion and adoption of Western values, practices and standards across sectors and including in health professional education. Promoters of globalization have drawn positive associations between openness to trade, increased income and improvements in health at the population level ${ }^{10}$. However, these positive aspects do not necessarily denote a reduction in inequalities ${ }^{10}$. In healthcare, globalization is a critical factor shaping 
current priorities and discourses. The influence, positive and negative, of globalization in healthcare is wide reaching. Examples in which globalization in healthcare plays a key role include the emergence of new (Zika and COVID-19) or re-emergence (Ebola) of communicable disease ${ }^{11}$. In the context of communicable diseases, globalization may be seen as a positive factor as advances in communication technology allows earlier identification, monitoring and global response to outbreaks. This was exemplified in the 2014 response to the Western Africa Ebola outbreak. Alternatively, a negative consequence of globalization is that, one can argue that advances in transportation and travel facilitate the spread of communicable disease as seen in the 2003 severe acute respiratory syndrome (SARS) outbreak ${ }^{12}$. Globalization may promote the development of global public goods such as generating standardized data on disease burdens and health system performance 6 . However, globalization is also a factor in the rise of noncommunicable diseases including cancer, diabetes and cardiovascular disease through, for example, trends in diet and tobacco use.

There are many unanswered questions regarding risks and unintended consequences associated with globalization in various fields including healthcare and medical education. Frenk et al, presenting the Lancet Global Commission on health professionals for a new century, articulated the urgent need for health professions curricular reform ${ }^{3}$. The reform should address the emerging "a slow-burning crisis in the mismatch of professional competencies to patient and population priorities because of fragmented, outdated, and static curricula producing ill-equipped graduates from under-financed institutions" ${ }^{3}$. However, as one considers these calls for reform, and begin developing and implementing global curricula, one must consider whether the nature of these calls for reform are perpetuating the Western medical discourse. The influence and impact of globalization and neocolonialism on health professional education or more specifically medical education will form the centre of the work in this dissertation.

\section{Neocolonialism and Medical Education}

In medical education, globalization is influencing curriculum development, accreditation, student selection and practice patterns of healthcare professionals $\mathrm{s}^{3,9,13}$. Medical education scholars have called attention to the concept of neocolonialism as it relates to international education efforts ${ }^{1}$. Neocolonialism in medical education is conceptualized as practices of exporting Western concepts and pedagogical methods without consideration of power relations ${ }^{14}$. There is implicit tension between global standards, including the generation and setting of global core competencies, with educational ideals oriented to respecting local diversity and local historical educational practices ${ }^{1,15}$. The concept of a 'universally global physician' who has a minimum set of essential competencies, particularly linked to Western notions of quality in health care, raises concern over whose voice, perspective and priorities are represented in the development of such recommendations and how this 
concept is related to broader economies of medical education (i.e. who garners material and symbolic benefit) ${ }^{5}$. While critical education scholars would support that physician competencies must reflect local socioeconomic and political contexts and local health needs ${ }^{14}$, how this is realized with the rising popularity of articulating core or universal skills is unclear. One is led to question the degree to which developing shared concepts in medical education is possible without the undue influence of Western values ${ }^{14}$.

Many areas in the 'Global South' continue to be influenced by their colonial histories ${ }^{6}$. In addition, Western priorities and science are at the core of many international medical training programs ${ }^{16}$. The philosophies that underpin the production of medical knowledge and ultimately health professional education require greater reflection and study. The power differentials in the Global North versus the Global South have been proposed to perpetuate Western or Euro-American beliefs and priorities at the expense of local knowledges ${ }^{9}$. In modern medical education, the connections between how knowledge is constructed and what counts as representation and bias become salient concerns when we set out to determine whose priorities are brought to the forefront and what mechanisms or practices perpetuate a dominant discourse. In the Canadian context, internationalization is growing in pace ${ }^{17}$. Internationalization refers to the "increasing mobility of students, faculty, and staff amongst and between countries; growing number of teaching and research partnerships outside of Canada; and updates to curriculum to equip students to "address complex local and global challenges in socially accountable ways."18 The CanMEDS framework, which articulates seven key abilities physicians require to effectively meet their patients' healthcare needs, is a commonly recognized example of the influence of Canada on the field of medical education globally. This framework has been adopted in over twenty-seven countries, within and outside physician training ${ }^{19}$. How this Canadian framework is conceptualized and actioned in diverse geopolitical and sociocultural areas is a rich topic for study. To contextualize the importance of these internationalization efforts one must first reflect on previous trends and influences in medical education curriculum development, particularly the way in which knowledge has been constructed and decisions were made.

\section{Critical Concepts in Curriculum Development}

As we strive for better alignment between health professional training and the needs of patients and the healthcare system one looks to curricula as both a potential source contributing to poor alignment, as suggested by Frenk et $\mathrm{al}^{3}$, and as a mechanism with which to foster positive change. I have chosen a critical approach to the work on global curriculum development presented in this dissertation because this approach provides an incisive tool to investigate how programs are working and to what effect, and to examine assumptions and explore power relationships. For the purposes of this dissertation a'global curriculum' is conceptualized as a text, which intends to use a common vocabulary and 
shared philosophy, and which describes an outcome, including competency items, that are intended to be applicable across nations. The text may also contain recommendations about curriculum delivery and assessment or other associated considerations. This work uses a critical lens to explore how power is conceptualized in the social processes of curriculum development as well as its influence of local fit and implementation. Applying the concept of 'critical consciousness, described as "a reflective awareness of the differences in power and privilege and the inequities that are embedded in social relationships" 20 affords the opportunity to look differently at curricular development which to some may appear on the surface formulaic and impartial. This can be illustrated using the popularized and frequently cited medical education curriculum development theory proposed by Kern et al. Kern et al, describe a six-step approach which may initially appear applicable and relevant regardless of context ${ }^{21}$. They describe a continuous cycle of (1) problem identification and general needs assessment, (2) targeted needs assessment, (3) goals and objectives, (4) educational strategies, (5) implementation and (6) evaluation and feedback. Kern et al describe four underlying assumptions to medical education curriculum design including (1) educational programs have aims and goals, (2) medical education professionals have an ethical obligation to meet learner, patient and societal needs, (3) medical educators should be held accountable for the outcomes of their interventions and (4) that using a logical and systematic approach to curriculum development will result in success ${ }^{21}$. This recipe style approach is appealing; as it appears readily actionable however at the core is social interactions and decisions. One questions, as with any social process, how biases, implicit or otherwise, may influence the process of curriculum development and the final product. This theory is one example from the large body of medical education publications that, often implicitly, portray medical education principles, practices, standards and guidelines as internationally applicable and accepted. Applying a critical analysis to curriculum development theory and practices may reveal gaps and unintended effects or biases in the development process. An understanding of these gaps and biases would seem important if we wish to realize Frenk et al's $s^{3}$ vision for alignment of curriculum with patient and health system needs.

Cultural hegemony is a term used to describe the power exerted by a dominant group to put forward one authoritative definition of culture and/or reality such that other groups accept it as a common understanding ${ }^{22,23}$. Development and deployment of curricula in medical education may be fraught with cultural hegemony ${ }^{4}$. Reflecting on Kern et al's approach to curriculum development, if one is to focus on the needs assessment aspect, one may question whose voice dominates this process? How can one ensure, as the target for curricula grows to a global scale, that a few powerful voices are not ultimately controlling the outcomes of such activities? Similar questions may be asked in the setting of Delphi processes ${ }^{24,25}$ or collaborative groups ${ }^{26}$ developing global curriculum standards. If the unequal distribution of privilege and power globally is acknowledged, ${ }^{4}$ 
then exploring the nature of global curricula with an anti-colonial framework becomes an important priority. The perspectives and results from such work may inform future curriculum development efforts and potentially impact the risks of perpetuating cultural hegemony and promoting neocolonialism. Attention and research are needed to explore whether or not the failure of previous curricular efforts to impart lasting change in a global context were related to post-colonial relationships ${ }^{3}$.

An anti-colonial approach further allows this work to explore "taken-for-granted norms and beliefs of a dominant culture and how individuals who do not fit into that culture are excluded." 27 Through this approach this work is able to interrogate "power configurations embedded in ideas, cultures and histories of knowledge production, validation and use ${ }^{28}$." Moreover, this approach allows for the critical examination of widely accepted perspectives which are often normalized in the international literature. One may then be able to shed light on opportunities to realize and integrate alternate viewpoints ${ }^{29}$. When considering curriculum development with a focus on global or core curricula in medical education the aspects of local-global tensions, seen through an anti-colonial lens, are important. Curriculum development and governance is often fraught with tension and what is considered 'core' can be heavily debated ${ }^{30}$. If curriculum theory teaches us that the fundamental step in design is defining 'locally relevant goals's1, these seem at odds with the concept of a globally accepted, standardized, curriculum. Standardization is a common theme in medicine which may in part be driven by the biomedical paradigm of evidence-based medicine. Proponents of standardization emphasize its role in improving quality in both training and patient care and facilitating movement of health professionals. However, standardization is a politically based enterprise which has been criticized for driving a loss of identify and social power and being vulnerable to implementation gaps ${ }^{32}$. Sefton describes the tensions inherent in meeting specific local community health needs while addressing international requirements or standards ${ }^{30}$. As one considers medical education curriculum development to achieve a global curriculum, considerations such as culture, power and the tension between local relevance and international standardization trends become relevant. One questions if traditional methods in medical education curriculum development adequately attend to these considerations and how anti-colonial perspectives may be applied. Three core concepts have been articulated in the context of medical education which are relevant to the work undertaken in this dissertation: homogenization, polarization, and hybridization ${ }^{33}$. Homogenization is the adoption of Western or international standards, the primacy of Western medical training, and the predominance of English language. Polarization refers to highlighting cultural distinctions, explicitly addressing local needs, and presenting locally-focused innovations. Finally, Hybridization refers to East-West partnerships, the adaptation of Western methodologies and the exchange of ideas from East to West. In the studies conducted 
in this dissertation the role of homogenization as a driving factor in global curriculum development is explored.

The practice of curriculum development and delivery in medical education, in Western contexts, has experienced several major reforms starting from the Apprenticeship-Based model (1765) and leading to the more recent clinical-presentation model (1991) $)^{34}$ and competency based medical education models $(2015)^{35}$. Shifts in these curricular trends have been driven by changes in the understanding of cognitive processes ${ }^{34}$ and facilitated by government and professional body influences. The publication of the Flexner report in $1910^{36}$ is considered a major turning point for North American health professions education. Although the Flexner report and the subsequent changes to medical education that followed are often considered positive by improving standardization and rooting medical training in analytic thinking ${ }^{13,37}$ there were other effects. Following the Flexner report there was a reframing of what was 'legitimate' in medical education and what followed were efforts of standardization, particular definitions of quality and homogenization. As such, there were a number of unintended consequences including limiting women's access to medical training ${ }^{16}$. In addition, the tensions between biomedicine and humanism can be traced to consequences of the Flexner report. This tension was an example of an unintended effect of the report and its interpretation and application to medical education ${ }^{13}$. Rational, analytic thinking, linked to the natural sciences, was central to Flexner's view of physician training ${ }^{37,38}$. With critical reflection on the seminal, historical works that have shaped our current, Western conceptualization of medical education one must also reflect on current paradigms and processes which may have similar unintended effects. As the 100 year anniversary of Flexner past, the calls for reform in medical education continue ${ }^{13,30}$. These calls describe a "fundamental redesign of the content of medical training ${ }^{\prime 13}$. While Cooke et $\mathrm{a}^{137}$ acknowledge such changes are challenging and may results in "turf battles" they state that "the challenge is not defining the appropriate content" but incorporation of content and faculty development ${ }^{37}$. One can argue that a critical, reflexive stance to curriculum development, its challenges and potential unintended effects are needed.

\section{Research Aims and Conceptual Lens}

The overall aim of this dissertation and the studies conducted are to explore, through critical approaches, the motivations behind and unintended effects of global curricula in medical education. This work applies a critical, anti-colonial lens to the work to investigate how various axes of power, including language, regionality, and gender influence the process and implementation of global medical curricula. Our work was inspired by the empirical work of anti-colonial scholars ${ }^{39}$, however I cannot claim to have realized a true anti-colonial analysis in this work. This is due to ontological, epistemological and axiological differences between the dominant literature in oncology and health professions studies 
which utilize anti-colonial theory. This dissertation was an opportunity to look for ways to introduce the oncology readership to these critical perspectives in a way that this message would be accepted by this audience. This work may be a step in bridging the differences in the literature of these two disciplines.

The field of critical theory as applied to curriculum design ${ }^{40,41}$ is particularly relevant to this work. There are differing forms of critical pedagogy, such as anti-colonial, feminist and antiracist approaches. What they all share is a specific focus on power relations and their effects in education. Critical educators are particularly attentive about the transmission and reproduction of problematic socio-cultural, political and economic relationships in the development and delivery of curricula. This work provides an opportunity to apply anti-colonial perspectives to curriculum design in oncology ${ }^{29}$ which has not been previously done. Given the increasing global demand for and focus on curriculum design, standardization and dissemination in this field, applying an anti-colonial perspective will provide a rich opportunity to explore the current process of curricular creation and transmission and its impact on local perspectives.

In this dissertation, the example of humanism in medicine is used to explore tangible challenges in the globalization and homogenization of medical curricula ${ }^{23}$. When considering the calls for curricula reform such as those by the World Health Organization $(\mathrm{WHO})^{42}$ and the Lancet Commission ${ }^{3}$ comments such as the need to nurture "health workers in the public service ethics, professional values and socially accountable attitudes requisite to deliver respectful care that responds to local needs and to population expectations $^{\prime \prime 2}$ are of interest. Realizing curricular reform is challenging in the current educational systems in which sub specialization, standardization, and technology integration impact humanism in care $^{43}$. Humanistic competencies, such as honesty, integrity, caring, compassion, altruism, empathy and respect ${ }^{44}$, might require better articulation in global curricula and may be integral to training professionals for future medical care as described in the preceding WHO reports. However, the way in which these humanistic qualities are conceptualized and prioritized, considered essential in for example Canadian medical care, require greater exploration and consideration in the context of global curricula. Such competencies are intrinsically linked to local culture and power relationships and are a rich area to review neocolonial effect. While the idea of humanism in medicine may appeal to health systems across the globe, what practices are experienced as humanistic will likely differ by context and culture. In a recent review of European medical education curricular trends Humanistic qualities were one area of controversy in differing geographic regions ${ }^{45}$. 


\section{Research context}

Oncology, or the branch of medicine which deals with the treatment of cancer, provides an interesting case to study the effects of neocolonialism in global curricular design. Non-communicable diseases (NCD) have surpassed communicable diseases as the major cause of death globally. The World Health Organization Sustainable Development Goal (SDG) 3, Good Health and Well Being, calls for a one third reduction in mortality from noncommunicable diseases, including cancer, by $2030^{46}$. Cancer is one of the most common NCDs and is increasing in incidence rapidly. In 2016, 138 countries were classified as low and middle income countries (LMIC) ${ }^{47}$. LMIC account for $56 \%$ of cancer diagnoses and $64 \%$ of cancer deaths around the world ${ }^{48}$. There exist major disparities in access to cancer care globally ${ }^{49}$. This is true for all aspects of cancer care from prevention activities, screening, diagnosis, treatment modalities of medical oncology, radiation treatment and surgery and supportive care, pain management, and end of life care. These disparities are linked directly to poorer outcomes including stage at diagnosis, chance of survival and quality of life following cancer treatment. The disparities are multifactorial including health systems infrastructure, treatment related resources (such as access to radiotherapy machines), lack of health human resources and supportive care and symptom management resources ${ }^{50}$. For example, radiotherapy is a key aspect of cancer care for both curative and palliative treatments ${ }^{51,52}$ however, despite this great need for radiotherapy many areas of the world have little or no access to radiotherapy ${ }^{53,54}$.

In 2011, the Union for International Cancer Control (UICC) formed a taskforce to address access to radiotherapy around the world. This taskforce was called "Global Task Force on Radiotherapy for Cancer Control" (GTFRCC). This taskforce quantified the need for global investment in radiotherapy infrastructure and demonstrated an economic advantage to the investment in radiotherapy services ${ }^{54}$. They concluded that equitable access to radiotherapy would save millions of lives as well as result in an economic advantage. Given these compelling arguments for investment in radiotherapy services the GTFRCC proposed five "calls to action":

1. Population based cancer control plans

2. Expansion of access to radiotherapy

3. Greater human resources for radiotherapy

4. Sustainable financing to expand access to radiotherapy

5. Align radiotherapy access with universal health coverage

Call to action three is of particular interest and concern to educators. Clearly, investing in radiotherapy physical infrastructure without addressing the need for health care professionals is futile and will not address societal needs. The WHO's Global Strategy on human resources for health stated in its recommendations "health systems can only 
function with health workers" ${ }^{\prime 42}$. In a review of current state and future projections a need for 12,149 radiation oncologists, 29,140 radiation therapists, and 9,915 medical physicists in low and middle income countries by 2020 was projected ${ }^{16}$. The GTFRCC model, extrapolating from current professional scopes of practice in the four core radiotherapy disciplines (radiation oncology, medical physicists, radiation therapists and nurses), has reported the need for 215,000 new health care professionals (HPC) by $2035^{54}$. The GTFRCC task force called for novel curricula, models of training and credentialing to address the need for over 200,000 health care professionals. However, the GTFRCC taskforce did not elaborate nor provide guidance on the content for such curricula nor how they may be developed.

Several agencies have previously proposed global curricular content and standardization for oncology ${ }^{26,55}$. The intention of these curricula was to provide standardized training for oncologists around the world. The agencies are based predominately in North America and Europe and the degree to which non-Western stakeholders are engaged in, or endorsing of, these curricula is unclear. It is these conditions that make oncology a pertinent case study for exploring the effects of neocolonialism in medical education.

\section{Research Questions and Approach}

To critically explore neocolonialism effects in the context of global oncology curricula, several critical questions were identified. Firstly, how are global medical curricula premised by stakeholder groups and what is their purported purpose? Secondly, whose voices are represented in consensus work to develop global oncology curricula? How are global oncology curricula conceptualized and implemented and what are their relationship to local contexts of power and culture? What are the factors that influence global oncology curriculum implementation? The international efforts to address the growing health human resource crisis in oncology afford us a unique opportunity to problematize our assumptions in global medical curriculum development. This opportunity allows for comment on curriculum development practices and the possibility to articulate recommendations which may positively impact future efforts.

\section{These issues raise 5 important research questions:}

1. What are the motivations and perceived premises for a global medical curriculum?

2. What global curricula exist for oncology? Who has developed them and what methods were used in their development?

3. What competencies are and are not included in the existing global curricula for oncology?

4. How are global oncology curricula conceptualized and implemented and what is their relationship to local contexts of power and culture?

5. What are the factors that influence global oncology curriculum implementation? 


\section{Dissertation Outline}

This dissertation is comprised of a series of studies designed to answer the five research questions outlined above. A summary of the remainder of the dissertation follows.

Chapter 2: In Chapter 2 we set out to understand the alignment of purpose with process in the development of global medical curricula including considering the socio-political differences of participants. Through a scoping review methodology we explore the challenges in creating such curricula and implications for local relevance. This chapter addresses research question 1 "What are the motivations and perceived premises for a global medical curriculum?".

Chapter 3: In this chapter we build on the considerations raised in Chapter 2 and focus on oncology as a case study. We conduct a systematic review to identify global oncology curricula and using an anti-colonial lens explore the issue of representation in global oncology curricula and alignment of purpose of these curricula and development methods. This chapter contributes to our understanding of research question 2 "What global curricula exist for oncology? Who has developed them and what methods were used in their development?".

Chapter 4: In Chapter 4 we perform an in-depth, framework analysis of the 17 global oncology curricula identified in Chapter 3. Through this analysis we explore the incorporation of humanistic competencies in global oncology curricula, their relationship to CanMEDS roles and how these priorities and associations may reproduce Western medical priorities. Chapter 4 addresses research question 3 "What competencies are and are not included in the existing global curricula for oncology?"

Chapter 5: As our work on chapters 2 to 4 progressed, addressing research questions 1 to 3 , it became clear there was a need to more deeply and richly explore how global curricula are conceptualized, challenges and perceived mitigating factors to these challenges. In Chapter 5 we problematize the concept and implementation of global oncology curricula and their relationship to local contexts of power and culture. This is achieved through indepth, one-on-one semi-structured interviews. Through iterative analyses the meaning and implication of the themes are discussed and refined to yield a conceptual analysis of the neo-colonial relationships reproduced and/or perpetuated through educational efforts to develop and implement global oncology curricula. Chapter 5 addresses research question 4 "How are global oncology curricula conceptualized and implemented and what is their relationship to local contexts of power and culture?"

Chapter 6: In Chapters 2 to 5 a recurring, emerging issue was the challenges of implementation of global oncology curricula. However, other than identifying 
implementation as an issue, data was not available about more specific aspects of these implementation challenges. In Chapter 6 we conduct an international, cross-sectional survey study focused on the European Core Curriculum for Radiation Oncology/

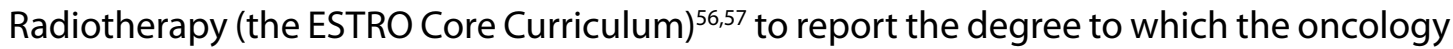
curricula has been implemented across Europe and to investigate the factors that influence curriculum implementation. Chapter 6 addresses research question 5 "What are the factors that influence global oncology curriculum implementation?"

Chapter 7: Chapter 7 summarizes and presents a discussion on how chapters 2 to 6 have addressed the research questions and will address the overall strengths and limitations of the work presented in this dissertation. A concluding review of how this work has advanced what is known in the development and implementation of global oncology curricula is provided. The dissertation ends with a discussion of possible next steps in this field of study.

\section{Reflexivity}

Bleakley et all define reflexivity as "a deeper process of inquiry into what values drive practices and activities in the first place and how activities are conceived, legitimated and executed through the interplay of identity, power and location"58. This is an opportunity to discuss how my beliefs, values and position may have influenced the work in this dissertation ${ }^{59}$. In this introduction chapter it is important to reflect about how my position as a healthcare professional, specifically as a radiation oncologist, and how my previous experience with education and global oncology shaped my interest in this topic. In addition, it is important to explore how my insider position both as an oncologist and a participant in the development of global curricula has afforded me both unique access and cognitive dissonance in a critical examination of global oncology curricula. It is important to acknowledge how my focus and insights were shaped through discussions with my supervisors and from what we learned from each project. I have approached the work in this dissertation with an empirical critical lens with the intent to provide information to deepen the understanding of understudied aspects of global curriculum development and potential unintended effects. This was an opportunity to give voice to what I perceive those engaged in this form of curricular development are already feeling.

I am a radiation oncologist at Princess Margaret Cancer Centre in Toronto, Canada. As a large comprehensive cancer centre Princess Margaret has many collaborations in clinical care and education round the world. I am also the Education Chair for the Canadian Association of Radiation Oncology and in this role represent Canadian Radiation Oncology on a number of global oncology education forums. In the Canadian context, I have been involved in curriculum redesign at the national level as my specialty prepared to implement competence by design $(\mathrm{CBD})^{35}$, the new national postgraduate training 
curriculum. This curriculum revision was a multi-year, social group process. Currently the first iteration of CBD curriculum in radiation oncology has been implemented. All of these prior experiences, culminating in my work on CBD at a national level led to my interest in educational process and representation at the global level. In parallel with this growing internal interest, there was a global effort to address the gaps in access to radiotherapy around the world which was published in 2015 called the Global Task Force on Radiotherapy for Cancer Control (GTFRCC) ${ }^{54}$. This publication, like others in medical and surgical oncology, highlighted a growing health human resource crisis for cancer care. This created a burning platform for a focus on ways to increase the number of oncologists and the role of global or core curricula were at the centre of these discussions.

I reflected over this growing focus on training at the global level and what I had experienced at Canadian national curriculum working groups for $\mathrm{CBD}^{35}$ in radiation oncology and the implications for the final produce of that process. It was at this time I realized I would benefit from an opportunity to continue my graduate studies at the doctoral level. My supervisors were instrumental in exposing me to the work of Edward Said and the concepts of neocolonialism in medical education. We found that little work in this area had focused on oncology and saw a need for exploration. Through my readings and the research team meetings I gained a deeper understanding of the methods and theoretical frameworks to interrogate and question the current, widely accepted, practices in curriculum development. As projects one to three were completed it became clear the need to explore more deeply the challenges and potential opportunities to overcome these challenges. Interviews were selected to access rich data on this topic. Project five, the final project, evolved from the desire to garner an understanding of implementation issues and considerations across a region with respect to a global or core curricula. The ESTRO Core Curriculum ${ }^{26,56}$ was selected as a case study for this purpose as I have professional connections to the educational group which afforded unique access to answer this research question. As the work in this dissertation evolved, I found myself more naturally questioning the decisions that go into selection of membership for curriculum development, the difficulties inherent in trying to ascertain if representation is enough to realize diverse, locally acceptable final products and ultimately what role global or core curricula can serve in increasing access to cancer care. I am aware of my unique position as both an 'insider' and an individual engaging in critical research. I am engaged in the system and processes that are the subject of the work in this dissertation. This provided me unique access to explore the research questions identified.

I see my position as a physician who works in an internationally recognized organization in Canada as a privileged position. As a Canadian, a country with a strong reputation in medical education, my voice may be more dominant in an international group setting. It 
has become clear to me as I move forward in my career the need to explicitly reflect on these positions of power and the unintended consequences they can lead to.

Finally, what became clear as we completed our work and submitted for publication, was the way in which specialties, specifically oncology, construct knowledge and what is considered comfortable to readers are in fact, limiting factors in moving the needle of understanding across disciplines. Oncology is steeped in the positivist paradigm and engaging in critical approaches for publication in oncology focused journals can be challenging. Where an education focused journal would really engage in a discourse on neocolonialism this topic was more challenging in oncology focused readership paradigms. As a research team we explicitly discussed these challenges, and reflected on the opportunity they afforded, as we shaped our papers. We hope our work has introduced the oncology readership to new concepts and has also contributed to the medical education communities understanding in our areas of study. 


\section{References}

1. Bleakley A, Brice J, Bligh J. Thinking the post-colonial in medical education. Med Educ. Mar 2008;42(3):266-270.

2. PG A. Education and neocolonialism. In: Ashcroft B GG, Tiffin H, ed. The post-colonial Studies Reader. London: Routledge; 2004:452-456.

3. Frenk J, Chen L, Bhutta ZA, et al. Health professionals for a new century: transforming education to strengthen health systems in an interdependent world. Lancet. Dec 04 2010;376(9756):1923-1958.

4. Zaidi Z, Verstegen D, Vyas R, et al. Cultural hegemony? Educators' perspectives on facilitating cross-cultural dialogue. Med Educ Online. 2016;21:33145.

5. Martimianakis MA, Hafferty FW. The world as the new local clinic: a critical analysis of three discourses of global medical competency. Soc Sci Med. Jun 2013;87:31-38.

6. Feachem RG. Globalization: from rhetoric to evidence. Bull World Health Organ. 2001;79(9):804.

7. Matowe L, Katerere DR. Globalization and pharmacy: a view from the developing world. Ann Pharmacother. May 2002;36(5):936-938.

8. http://wrap.warwick.ac.uk/2010/1/WRAP_Scholte_wp10902.pdf. What is Globalization? The Definitional Issue - Again. In: Scholte, ed2002.

9. Bhandal T. Ethical globalization? Decolonizing theoretical perspectives for internationalization in Canadian medical education. Canadian Medical Education Journal. 2018;9(2):e33-45.

10. Dollar D. Is globalization good for your health? . Bulletin of the World Health Organization : the International Journal of Public Health 2001. 2001;79(9):827833.

11. Girard GRP-M. Toward a global health approach: lessons from the HIV and Ebola epidemics. Globalization and Health. 2018;14:114.

12. McKibbin J-WLaWJ. Globalization and Disease: The Case of SARS. In: https://www.brookings. edu/research/globalization-and-disease-the-case-of-sars/, edTuesday, May 20, 2003.

13. Ho MJ, Abbas J, Ahn D, et al. The "Glocalization" of Medical School Accreditation: Case Studies From Taiwan, South Korea, and Japan. Acad Med. Dec 2017;92(12):1715-1722.

14. Karle H, Christensen L, Gordon D, et al. Neo-colonialism versus sound globalization policy in medical education. Med Educ. Oct 2008;42(10):956-958.

15. Whitehead CR. On gunboats and grand pianos: medical education exports and the long shadow of colonialism. Adv Health Sci Educ Theory Pract. Mar 2016;21(1):1-4.

16. Hodges B. The many and conflicting histories of medical education in Canada and the USA: an introduction to the paradigm wars. Med Educ. Jun 2005;39(6):613-621.

17. Stein S AV, Bruce J, Suša R. Towards different conversations about the internationalization of post- secondary education. . Comp Int Educ. . 2016;45(1):2-18.

18. Internationalization at Canadian universities: quick facts. Accessed September 20, 2019; 2014.

19. Frank JR SL, Sherbino J, editors. CanMEDS 2015 Physician Competency Framework. Ottawa: Royal College of Physicians and Surgeons of Canada; 2015.

20. Kumagai AK, Lypson ML. Beyond cultural competence: critical consciousness, social justice, and multicultural education. Acad Med. Jun 2009;84(6):782-787. 
21. Patricia Thomas DK, Mark Hughes and Belinda Chen. Curriculum Development for Medical Education. Baltimore: JOhns Hopkins University Press; 2016.

22. Gramsci A. Further selections from the prison notebooks. Minneapolis: University of Minnesota Press; 1995.

23. Borg C BJ, Mayo P. Gramsci and Education. Lanham: Rowman \& Littlefield; 2002.

24. Tam VC, Ingledew PA, Berry S, et al. Developing Canadian oncology education goals and objectives for medical students: a national modified Delphi study. CMAJ Open. Jul-Sep 2016;4(3):E359-E364.

25. Powell C. The Delphi technique: myths and realities. J Adv Nurs. Feb 2003;41(4):376-382.

26. Eriksen JG, Beavis AW, Coffey MA, et al. The updated ESTRO core curricula 2011 for clinicians, medical physicists and RTTs in radiotherapy/radiation oncology. Radiotherapy and oncology: journal of the European Society for Therapeutic Radiology and Oncology. 2012;103(1):103-108.

27. Verdonk P, Abma T. Intersectionality and reflexivity in medical education research. Med Educ. Aug 2013;47(8):754-756.

28. George J. Sefa Dei AA. The Power of Social Theory: The Anti-COlonial Discursive Framework. Journal of Educational Thought. 2001;35(3):297-323.

29. Daneil Hollenberg LM. Epistemological challenges to integrative medicine: An anti-colonial perspective on the combination of complementary/alternative medicine with biomedicine. Health Sociology Review. 2010;19(1):34-56.

30. Sefton AJ. New approaches to medical education: an international perspective. Med Princ Pract. Sep-Oct 2004;13(5):239-248.

31. J B. Enhancing teaching through constructavist alignment. Higher Education. 1996;32:347-364.

32. Seftan Timmermans RA. Objectification, standardization, and commodification in health care: A conceptual readjustment. Social Science \& Medicine. 2009;69:21-27.

33. Gosselin K, Norris JL, Ho MJ. Beyond homogenization discourse: Reconsidering the cultural consequences of globalized medical education. Med Teach. Jul 2016;38(7):691-699.

34. Papa FJ, Harasym PH. Medical curriculum reform in North America, 1765 to the present: a cognitive science perspective. Acad Med. Feb 1999;74(2):154-164.

35. Canada RCoPaSo. Competency By Design. http://www.royalcollege.ca/rcsite/cbd/competence-by-design-cbd-e. 2019;Accessed October 22, 2019.

36. Flexner A. Medical Education in the United States and Canada: A Report to the Carnegie Foundation for the Advancement of Teaching 1910.

37. Cooke M, Irby DM, Sullivan W, et al. American medical education 100 years after the Flexner report. N Engl J Med. Sep 28 2006;355(13):1339-1344.

38. Duffy TP. The Flexner Report $\otimes 100$ Years Later. YAIE JOURNAI OF BiOIOGY AND MEDiCiNE 2011;84(f):269-276.

39. Carlson E. Anti-colonial methodologies and practices for settler colonial studies. Settler Colonial Studies. 2017;7(4):496-517.

40. Susan Adler JG. Critical Theory as a Foundation for Methods Courses Journal of Teacher Education. 1986;37(4). 
41. Graham J, Dornan T. Power in clinical teachers' discourses of a curriculum-in-action. Critical discourse analysis. Adv Health Sci Educ Theory Pract. Dec 2013;18(5):975-985.

42. WHO. Global Strategy on human resources for health: Workforce 2030. In: Organization WH, ed: Geneva, Switzerland 2016.

43. Martimianakis MA, Michalec B, Lam J, et al. Humanism, the Hidden Curriculum, and Educational Reform: A Scoping Review and Thematic Analysis. Acad Med. Nov 2015;90(11 Suppl):S5-S13.

44. Cohen JJ. Viewpoint: linking professionalism to humanism: what it means, why it matters. Acad Med. Nov 2007;82(11):1029-1032.

45. AMEE. Curriculum Trends in Medical Education in Europe in the 21 st Century. https://amee. org/getattachment/amee-initiatives/medine-wp5.pdf. Accessed December 27, 2017.

46. World Health Organization. Sustainable Development Goals. https://www.who.int/sdg/en/. Accessed December 27, 2017.

47. The World Bank. https://datahelpdesk.worldbank.org/knowledgebase/articles/906519-worId-bank-country- and-lending-groups. Accessed December 27, 2017.

48. Ferlay J, Soerjomataram I, Dikshit R, et al. Cancer incidence and mortality worldwide: sources, methods and major patterns in GLOBOCAN 2012. Int J Cancer. Mar 01 2015;136(5):E359-386.

49. Abdel-Wahab M, Bourque JM, Pynda Y, et al. Status of radiotherapy resources in Africa: an International Atomic Energy Agency analysis. Lancet Oncol. Apr 2013;14(4):e168-175.

50. Knaul FM, Farmer PE, Bhadelia A, et al. Closing the divide: the Harvard Global Equity Initiative-Lancet Commission on global access to pain control and palliative care. Lancet. Aug 22 2015;386(9995):722-724.

51. Borras JM, Lievens $Y$, Dunscombe $P$, et al. The optimal utilization proportion of external beam radiotherapy in European countries: An ESTRO-HERO analysis. Radiother Oncol. Jul 2015;116(1):38-44.

52. Rosenblatt $E$, Barton $M$, Mackillop W, et al. Optimal radiotherapy utilisation rate in developing countries: An IAEA study. Radiother Oncol. Jul 2015;116(1):35-37.

53. PACT I-. Advisory Group on increasing access to Radiotherapy Technology in low and middle income countries (AGaRT).http://cancer.iaea.org/agart.asp(Accessed June 272017 ).

54. Atun R, Jaffray DA, Barton MB, et al. Expanding global access to radiotherapy. Lancet Oncol. Sep 2015;16(10):1153-1186.

55. IAEA Syllabus for the Education and Training of Radiation Oncologists. 2009(Training Course Series No. 36). http://www-pub.iaea.org/MTCD/Publications/PDF/TCS-36_web.pdf.

56. Benstead K, Lara PC, Andreopoulos D, et al. Recommended ESTRO Core Curriculum for Radiation Oncology/Radiotherapy 4th edition. Radiother Oncol. Sep 52019.

57. Eriksen JG, Beavis AW, Coffey MA, et al. The updated ESTRO core curricula 2011 for clinicians, medical physicists and RTTs in radiotherapy/radiation oncology. Radiother Oncol. Apr 2012;103(1):103-108.

58. Alan Bleakley JBaJB. Medical Education for the Future: Identity, Power and Location. Vol New York: Springer; 2011.

59. Ramani S, Konings KD, Mann K, et al. A Guide to Reflexivity for Qualitative Researchers in Education. Acad Med. Aug 2018;93(8):1257. 
CHAPTER 2

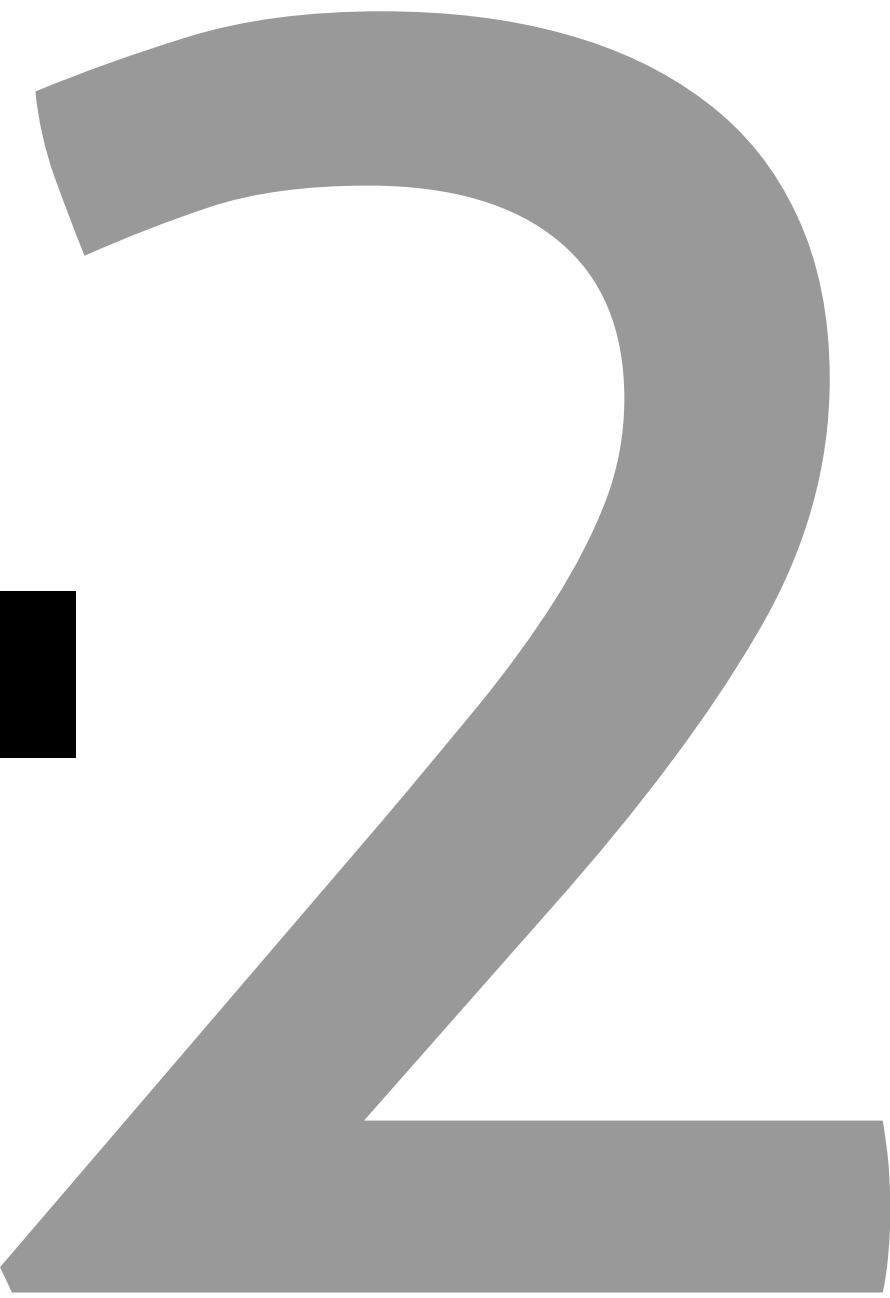




\section{Motivation and Challenges in the Development of Global Medical Curricula: A scoping review}

Accepted for publication: Giuliani M, Martimianakis M, Broadhurst M, Papadakos J, Fazelad R, Driessen E, Frambach J. Motivation and Challenges in the Development of Global Medical Curricula: A scoping review. Accepted to Academic Medicine December 2019. 


\section{Purpose}

Medical education is faced with mounting calls to develop global standards for training. However, the process of development and application of global standards are not well understood. The aim of this scoping review is to understand the motivations for the construction of global curricula, to summarize approaches that have been taken to create these curricula, and to understand the perceived premises for the construction of these curricula.

\section{Methods}

This scoping review involved a comprehensive search strategy of papers published from 1998 to 2018. There were no language restrictions. Two investigators independently reviewed publications for eligibility. Demographic data was abstracted from publications and summarized. The motivation for creating the curricula and the challenges were coded and summarized.

\section{Results}

137 publications met inclusion criteria. Most curricula were developed by a social group process (such as committee) or a Delphi process. The purpose for creating global medical curricula was most commonly to define speciality specific standards, to harmonize training standards, or to improve the quality of training. The most common challenges were intercountry variation including differences in the health care system, the operationalization of medical training and socio-cultural differences, the need for a multi-stakeholder approach and implementation.

\section{Conclusions}

The challenges of intercountry variation, the need for multi-stakeholder approaches, and curriculum implementation need to be considered if concerns of relevance are to be addressed. Such considerations undoubtedly impact uptake of the curricula and can only be addressed by explicit effort to make global curricula applicable to the realities of diverse health care sectors. 


\section{Introduction}

Medical education is faced with mounting calls to develop global curricula for training. ${ }^{1,2}$ These calls run in parallel with overall globalization developments in the field of health care including pandemics, the rise of non-communicable diseases and the social determinants of health, such as tobacco use. ${ }^{3}$ However, the real-world implications of global medical curricula, for example, local applicability, implementation and impact, are not well understood. ${ }^{4}$ The influence of such curricula in reproducing the dominance of the Western biomedical model is a concern to scholars following the loss of Indigenous and context specific health practices. ${ }^{5,6}$ In addition, the fit of these curricula within the local medical health care system is a potential challenge given the diversity of health care globally. This is important to understand as outdated or ill-suited curricula can lead to gaps in clinical care. ${ }^{7}$ The calls for global standards in medical education, including curricula, originate from influential educational entities such as the World Federation of Medical Education (WFME) and the Institute for International Medical Education (IIME). The World Federation of Medical Education, founded in 1972, is an international body which aims to promote quality improvement in medical education and has a current focus on accreditation and maintaining the World Directory of Medical Schools. ${ }^{1}$ The WFME has developed and disseminated standards in medical education from undergraduate through to continuing medical education. ${ }^{8}$ The WFME provides a curriculum development framework that can then be modified locally. The Institute for International Medical Education, founded in 1999, has the goal of developing global minimal essential requirements for physicians around the world. ${ }^{2}$ At a regional level, Europe has several initiatives to harmonize training standards including the Bologna Process ${ }^{9}$ and the European Credit Transfer System (ECTS). The ECTS addresses training in all disciplines and aims to facilitate recognition of training within Europe to promote quality training and the free movement of people. ${ }^{10}$ However, it is not a system that specifies curricular requirements. Specific to medical education, there is the European Union of Medical Specialists (UEMS), which represents over 50 medical disciplines and 37 countries in Europe. The mission of the UEMS is to"set standards for high quality healthcare practice that are transmitted to the Authorities and Institutions of the EU [European Union] and the National Medical Associations stimulating and encouraging them to implement its recommendations".11 Collectively, these internationally recognized organizations in education have, over the past two decades, made efforts to accomplish their goals of global standards in medical education including producing global curricula.

The impetus for creating global curricula in medical education is multifactorial. They are purported to have benefits including improving the quality of training, promoting individual-freedoms by promoting free-movement of people and mitigating gaps in health human resources. ${ }^{12}$ However, the development of curricular content is a social process that involves individuals with diverse values, histories, culture and varying levels of power. ${ }^{13}$ While there are many reasons to embark on the creation and revision of medical curricula, 
the most persuasive argument is often providing better education and by extension better patient care. It has also been proposed that curriculum development and renewal can be an opportunity for building strategic networks and bringing people and ideas together. ${ }^{13}$ This type of social networking is appealing if it promotes the sharing and the integration of knowledge and technologies from different sectors, and is a source of positive growth for all parties. However, proposing curricular solutions to address global challenges in medical education is mired in power dynamics and requires reflection and humility ${ }^{14}$ given the diversity of perspectives and local contexts represented in such discussions. Global medical curricula are vulnerable to being overly specific in their articulation of requirements, akin to a shopping list of competencies, which can remove the aspiration for innovation and excellence. ${ }^{15}$ In addition, they may lack a critical understanding of local cultural and/or historical norms which are critical to the delivery of health care and therefore medical training. ${ }^{14}$ In a previous publication, we identified a dominance of Western authors has been identified in the context of global oncology curricula. ${ }^{16}$ It is not known if the dominance of the Western perspective is occurring in global medical curricula in other disciplines outside of oncology. Understanding the stated purpose and voices represented in the construction of existing global medical curricula may yield insights into strengths and gaps to inform future curricular efforts of this kind. The aim of this scoping review is to understand the motivations for the construction of global curricula, to summarize the approaches that have been taken to create these curricula and to understand the perceived premises for the construction of these curricula. We aim to consider the alignment of purpose with process in the development of global medical curricula including considering the socio-political differences of participants.

\section{Methods}

We conducted a scoping review to understand and summarize existing efforts to create global medical curricula using the 5-step methodology of Arksey et al. ${ }^{17}$ We selected a scoping review methodology as it would allow a systematic mapping of the existing work in global medical curricula including the articulation of trends, facilitate comparison and identify gaps. ${ }^{18}$ The research team, including an information specialist (RF), constructed a comprehensive search strategy. The proposed search strategy was peer reviewed by information specialists prior to beginning the search. The following databases were searched to identify relevant papers published between 1998 to 2018: Medline, MEDLINE ${ }^{\circledR}$ Epub Ahead of Print and In-Process \& Other Non-Indexed Citations, EMBASE, Cochrane Central Register of Controlled Trials, Cochrane Database of Systematic Reviews, and PsycINFO, all from the OvidSP platform; CINAHL, and ERIC from EBSCOhost; Scopus from ELSEVIER; African Index Medicus (AIM), and LILACS (results in Spanish). Where available, both subject headings and text words were used to maximize search results and account for global linguistic variations. The search terms included: facult ${ }^{*}$, educat ${ }^{*}$, graduat ${ }^{*}$, postgraduate ${ }^{*}$, residen ${ }^{*}$ or clerkship ${ }^{*}$ for medical education; curricul*, program*, ${ }^{*}$ rain*, 
core $^{*}$, standard ${ }^{*}$ for curriculum and global $^{*}$, universal ${ }^{*}$, multi-national ${ }^{*}$, worldwide* for global. There were no language restrictions. The time span on 1998 to 2018 was selected based on a previous study ${ }^{16}$ where the proliferation of efforts in the area of global curricula was focused on the last 20 years. See Supplementary File 1.

\section{Inclusion and Exclusion Criteria}

The inclusion and exclusion criteria were applied by two independent researchers (MG and $M B)$. We resolved discordance through discussion to reach consensus regarding inclusion or exclusion. We included publications if they were physician focused, and related to any phase of medical education (e.g. undergraduate, postgraduate, continuing medical education), if they contained a global or regional ( $>1$ country) curricula or discussed the concept of global/regional/core curricula. Publications were excluded if they were intended for non-physician professions, focused on patient or caregiver education, were curricula on Global Health, surveys of practice or on the topic of International Medical Graduates.

\section{Data Abstraction and Analysis}

The following data was extracted by MG and MB from each publication: publication year, medical specialty that was the topic or focus of the publication, phase of medical training referred to in the publication (for example, postgraduate), number of authors on the publication, and the countries and geographic region of authors on the publication. The explicitly stated purpose for creating global medical curricula was recorded if present in the publications. For this analysis, 'purpose' was defined as the explicit statement in the publication regarding the reason for creating global medical curricula. For publications which were actual curricular documents, the methods used to develop the curricula was recorded. Descriptive statistics were used to summarize these demographic data.

As part of our analysis, we also sought to understand if there was transmission of Western ideologies through the development and implementation of global medical curricula. The research presented in this paper builds on previous research conducted by the authors. ${ }^{16}$ In past work, we identified that exploring the approach taken in the development of curricula would yield important information on the power relations between resource rich and resource poor states. Thus, to capture the rationale and approach we coded the documents deductively to ascertain: the stated purpose, the methods used for the development of the curricula, the stated motivation (theoretical benefits or driving factors) and the reported challenges. We also coded for geographic area of participating curriculum developers. Together, the data we coded for allowed us to capture patterns related to power relations. We believe this type of analysis, currently absent in the literature, will advance the current approaches to developing global medical curricula 
because it makes gaps in representation visible and foregrounds potential unintended aspects of promoting global curricula.

A subset of articles was initially coded by MG. The coding approach and structure was reviewed and discussed by the authors (MG, JF, ED, TM, JP) until coding was consolidated into core areas of focus by mutual agreement. The data was then reanalyzed and summarized by MG and MB. The coding was completed using NVivo software version 11 (QSR International Pty Ltd. Version 11, Victoria, Australia). Following coding of the entire dataset, the analysis and interpretation of results was refined through multiple research meetings between MG and the other authors (JF, ED, TM, JP).

\section{Results}

\section{Publication Demographics}

The initial search resulted in 18,684 papers. After the removal of duplicates, title scan and review of the abstracts, 312 articles remained and underwent full text review. Of the 312 articles, 137 met inclusion criteria and formed the basis of this analysis $s^{4,9,10,15,19-151}$. See Figure 1.

The region of origin of the authors was most frequently Europe $(n=58 ; 42 \%)$, followed by North America ( $n=18 ; 13 \%)$ and $49(36 \%)$ of publications were from authors from more than one region. Most, $98 \%$, were published in English. The mean number of authors on the publications was 8 (range 1 to 145). Seventy-eight (57\%) articles were published between 2011 and 2018 and most ( $n=81 ; 59 \%$ ) were focused on postgraduate medical education. Medicine and medical subspecialties were the most common speciality represented and accounted for 52 (38\%) publications. A publication may have articulated more than one purpose for creating global medical curricula. We identified 166 purpose statements in the 137 publications. The explicit purpose of creating global medical curricula was most commonly to define speciality specific standards ( $n=50 ; 30 \%)$, to harmonize training standards $(n=38 ; 23 \%)$, or to improve the quality of training $(n=31 ; 23 \%)$. See Table 1 .

\section{Challenges with Global Medical Curricula}

The most common challenges articulated in the publications were intercountry variation $(n=27 ; 20 \%)$ and the need for a multi-stakeholder approach $(n=6 ; 4 \%)$. In addition, curricular implementation was a challenge and recommendations on implementing global medical curricula were only identified in a minority of the publications $(n=20$; 15\%). See Table 2.

The articulation of how intercountry variation impacts global curricula was captured in three main areas. The first was differences in the health care system. For example, the 

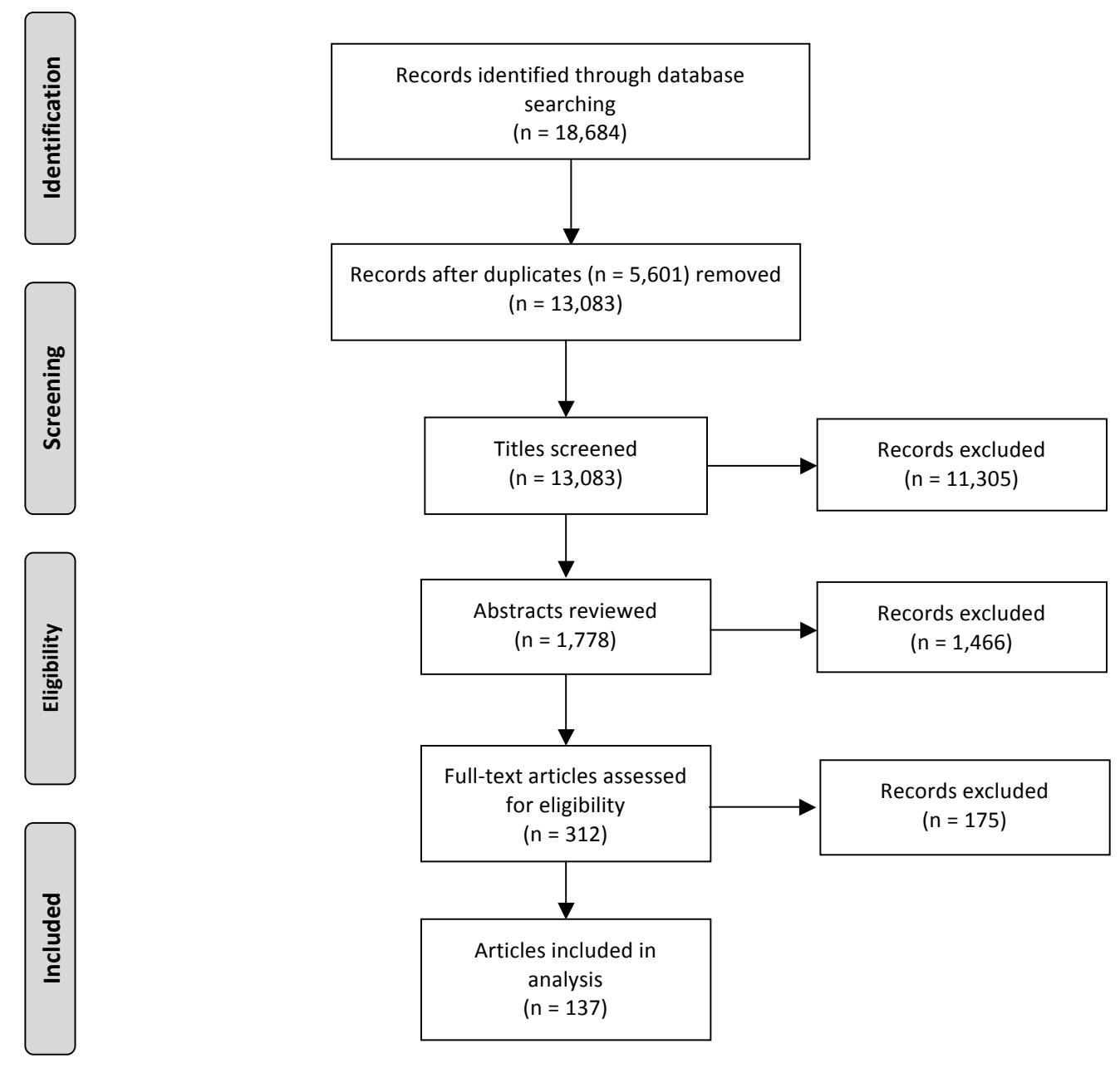

Figure 1: PRISMA 2009 Flow Diagram

concept of the link between the content of the curricula and the health system in one document was articulated as "this [content] is not only depending on national traditions, but mainly on the way neurology is practiced and how health system structures are used", ${ }^{\prime 42}$ Other concerns with intercountry variation included the operationalization of medical training including the duration of training ${ }^{37}$ and socio-cultural differences including "widespread cultural and religious diversities and positioning of the doctor within society".85 Intercountry variation was presented as a positive or a negative challenge. For example, Hodges et al. propose "it is time to study and embrace differences and discontinuities in goals, practices and values that underpin medical competence in different countries", 11 while others view this as a limitation on achieving uniformity, stating "medical curriculum differs greatly worldwide in their content, thus, levels of professional competences acquired by graduates of medical schools varies across the globe. Consequently, it becomes difficult to get a uniform global physician".94 Finally, intercountry variation, and the tension between the universal and the local priorities were 
Table 1: Publication Characteristics

\begin{tabular}{|c|c|c|}
\hline & & Number (\%) \\
\hline \multicolumn{3}{|l|}{ Year of Publication } \\
\hline & $1999-2010$ & $59(43 \%)$ \\
\hline & 2011-2018 & $78(57 \%)$ \\
\hline \multicolumn{3}{|l|}{ Phase of Training } \\
\hline & Undergraduate MD & $23(17 \%)$ \\
\hline & Postgraduate MD -Residency & $78(57 \%)$ \\
\hline & Postgraduate MD -Fellowship & $3(2 \%)$ \\
\hline & Continuing Medical Education & $7(5 \%)$ \\
\hline & Mixed Level & $24(18 \%)$ \\
\hline & Other & $2(1 \%)$ \\
\hline \multicolumn{3}{|l|}{ Number of Authors } \\
\hline & 1 to 3 & $56(41 \%)$ \\
\hline & 4 to 9 & $56(41 \%)$ \\
\hline & $\geq 10$ & $24(18 \%)$ \\
\hline & Unknown & $1(1 \%)$ \\
\hline \multicolumn{3}{|l|}{ Region of Authors } \\
\hline & Africa & $3(2 \%)$ \\
\hline & Asia & $4(3 \%)$ \\
\hline & Oceania & $2(1 \%)$ \\
\hline & Europe & $58(42 \%)$ \\
\hline & Latin America & $2(1 \%)$ \\
\hline & North America & $18(13 \%)$ \\
\hline & Multiple regions & $49(36 \%)$ \\
\hline & Unknown & $1(1 \%)$ \\
\hline \multicolumn{3}{|c|}{ Number of Regions in Authorship } \\
\hline & 1 & $87(64 \%)$ \\
\hline & 2 & $31(23 \%)$ \\
\hline & 3 & $10(7 \%)$ \\
\hline & 4 & $3(2 \%)$ \\
\hline & 5 & $4(3 \%)$ \\
\hline & 6 & $1(1 \%)$ \\
\hline & Unknown & $1(1 \%)$ \\
\hline \multicolumn{3}{|l|}{ Publication Language } \\
\hline & English & $134(98 \%)$ \\
\hline & Other & $3(2 \%)$ \\
\hline \multicolumn{3}{|l|}{ Medical Speciality } \\
\hline & Medicine \& Medical Subspecialties & $52(38 \%)$ \\
\hline & Pediatrics \& Pediatric Subspecialties & $1(1 \%)$ \\
\hline & Psychiatry \& Psychiatry Subspecialties & $5(4 \%)$ \\
\hline & Radiology and Nuclear Medicine & $9(7 \%)$ \\
\hline & Surgery and Surgical Subspecialties & $15(11 \%)$ \\
\hline & Medical Education & $27(20 \%)$ \\
\hline & Other & $28(20 \%)$ \\
\hline
\end{tabular}


Table 1 (continued)

\begin{tabular}{|c|c|}
\hline Declared purpose of creating global medical curricula ${ }^{a}$ & Number $(n=166)^{a}$ \\
\hline Define Common Standards & $50(30 \%)$ \\
\hline Harmonization & $38(23 \%)$ \\
\hline Improve Quality/Safety & $31(19 \%)$ \\
\hline Promote and Define a Speciality & $24(14 \%)$ \\
\hline Physician Mobility & $16(10 \%)$ \\
\hline Address Health Human Resource Shortages & $5(3 \%)$ \\
\hline Reduce Inequalities & $1(1 \%)$ \\
\hline Cost Effectiveness & $1(1 \%)$ \\
\hline
\end{tabular}

${ }^{\text {a }}$ A publication may have articulated more than one purpose for creating global medical curricula.

Table 2: Challenges and Motivation Associated with Global Medical Curricula

\begin{tabular}{ll}
\hline Challenges & Number of publications \\
\hline Intercountry variation & 27 \\
\hline Curriculum Implementation & 20 \\
\hline Multi-stakeholder approach & 6 \\
\hline Health professional shortages & 4 \\
\hline Resource constraints & 4 \\
\hline Other & 11 \\
\hline Perceived Motivation/Benefits & \\
\hline Physician mobility & 9 \\
\hline Improve quality & 6 \\
\hline Harmonization & 5 \\
\hline Other & 3 \\
\hline
\end{tabular}

identified as a factor in the success of the implementation of global curricula. It was, for example, argued that "[d]evelopment of these training programs is multifaceted and must take into account the specific needs of the recipient country in order to be successful".?2

Recommendations on implementing global medical curricula were only discussed in a minority $(n=20 ; 15 \%)$ of the 137 publications. Among those that addressed implementation, the most frequent comment was the need for local adaptation to facilitate implementation. Authors who have made global medical curricula implementation recommendations have articulated that "flexibility has to be important, otherwise parts of Europe may be unable to offer training..."${ }^{178}$ in alignment with the proposed curricula and that implementation of "training standards and curricula in local contexts will be best guided by regionally determined policies in regard to some key implementation issues". 129 The role of global curricula was described as a roadmap in one article; "[a] core syllabus should not dictate when or how the content is to be delivered. Its value is simply to provide a helpful roadmap for related educational journeys".38 Other implementation considerations included having appropriate assessment tools to evaluate educational outcomes, the tension between the priorities of academic institutes and the health care system, ${ }^{131}$ and barriers including 
financial, political and cultural; "initially, the implementation of the project of universal medical curriculum will face lots of cultural, political and sociological difficulties".85 These considerations resonate with the next challenge, the importance of a multi-stakeholder approach.

The need for a multi-stakeholder approach challenged the dominant paradigm of using social group processes or Delphi methodology as the most appropriate methods for developing global curricula. In this review, the majority, $78 \%(n=107)$, of curricula were created using these methods. Authors advocating for a multi-stakeholder approach propose that "expert consensus alone is not the most appropriate way to define the professional competencies required for clinical practice" ${ }^{1106}$ and question "is there a more accurate way of capturing competency beyond expert consensus?". 138 They allude to the need to extend these consultations beyond the expert core to stakeholders including those locally implementing the curricula.

Incongruence between Western priorities and local culture, ${ }^{85,91,122,149}$ suppression of traditional approaches ${ }^{91}$ and reducing diversity ${ }^{91}$ were also challenges which were identified by members of the global curricula teams. Authors have articulated "a risk of reduction of diversity and a suppression of traditional knowledge or approaches, by dominant groups or countries..." ${ }^{91}$ and that "while there are quite distinct cultural aspects to medicine, medical education tends to be blind to these differences and act as a 'culture of no culture ${ }^{\prime \prime \prime 91}$. They argued for critical reflection during the curriculum design process to determine what perspectives are present in the consensus processes and if certain competency areas are underrepresented due to this challenge between the universal and diversity. Authors argued that programs should counter Western dominance by "tak[ing] into consideration the socioeconomic and cultural framework of the local community when designing curricula"122 and to be mindful that there may exist "incompatible cultural attitudes like individualism, difficulty in getting involved in discussions, and exaggerated tendency to be outspoken in some of their [non-West] students" ${ }^{\prime \prime 5}$ when moving discussions and curricula from Western to non-Western contexts.

\section{Motivation for Creating Global Medical Curricula}

In addition to the explicit purpose for creating global medical curricula the documents $(n=137)$ also described theoretical benefits or driving factors for creating global medical curricula and we have coded these under 'Motivation'.

The most frequently cited motivation behind global medical curricula was promoting physician mobility $(n=9 ; 7 \%)$ for employment. Global curricula were promoted as making movement to different areas easier by assisting regulatory bodies in credentialing physicians in different regions, as exemplified in the following excerpt, "the certification or 
credentialing of vascular and endovascular surgeons who have all achieved the expected competencies agreed to internationally allows regulatory bodies to develop consistent approaches between countries."76

Quality ( $n=6 ; 4 \%$ ) was the next most frequently cited motivation for creating global medical curricula. The quality narrative focused either on the quality of patient care, the quality of the training programs, or the role of quality training in realizing high-quality patient care. For example Besso et al. articulate "[h]armonizing educational outcomes and strengthening processes of training and accreditation facilitates freedom of movement of specialists, and enhances the quality of care they provide worldwide".43 Others describe the role of these curricula in "meeting demands of the public for guarantees of graduate competence and cost-effectiveness of their training".38

\section{Methods for Developing Global Medical Curricula}

A subset of the 137 publications reported actual curricular documents ( $n=67 ; 49 \%$ ). These 67 curricula were analyzed to determine the methodology used for the creation of the global medical curricula. The most common methods used were a social group process (such as committee or expert panel) $(n=30 ; 45 \%)$ or a Delphi/Modified Delphi process ( $n=22 ; 33 \%)$. See Table 3.

Table 3: Methodology for the Creation of Global Medical Curricula

\begin{tabular}{ll}
\hline Methods & Number (67) \\
\hline $\begin{array}{l}\text { Social group process } \\
\text { (expert panel, task force, working group etc.) }\end{array}$ & $30(45 \%)$ \\
\hline Delphi/Modified Delphi & $22(33 \%)$ \\
\hline Literature Review & $2(3 \%)$ \\
\hline Mixed Methods & $6(9 \%)$ \\
\hline Survey & $3(4 \%)$ \\
\hline Other & $4(6 \%)$ \\
\hline
\end{tabular}

\section{Discussion}

In this study, we have demonstrated that the majority of publications, which either describe or comment on global medical curricula, originate from Western regions, namely Europe, the U.S and Canada. This is an important finding as a predominance of Western discourse in the literature on global medical curricula may suppress important views from non-Western stakeholders. This, in-turn, may limit the utility of these curricula and contribute to an ongoing imbalance between curricular content and health system needs. ${ }^{7}$ We have described the narrative on the challenges with global medical curricula that centered on intercountry variation, the need for a multi-stakeholder approach and implementation considerations. 
The usefulness of global medical curricula has not been assessed once they have been implemented. ${ }^{4}$ It has been recommended that global curricula should be developed in consultation and collaboration with those stakeholders who will ultimately implement the curricula. In addition, Bandaranayake articulates the need to maintain societal relevance should take precedence over standardization. ${ }^{38}$ These are significant challenges and further studies to improve both the understanding and the approach to the development and implementation of global medical curricula are needed. Our data do articulate an awareness of the need for diversity of input and the importance of engaging the stakeholders who will be implementing the curricula. However, international standards, despite efforts in the planning stage, can still reflect Western standards ${ }^{152}$ and whether diversifying stakeholder input alone is able to mitigate this problem is uncertain. An important example of the concern of Western values driving the standardization movement is articulated in a commentary on the Educational Commission for Foreign Medical Graduates (EGFMC) policy that in 2023, international medical graduates (IMGs) must have graduated from a formally accredited medical school to receive ECFMG certification. This policy has the potential to impact the physician workforce, not only in the United States, but in other countries who may have an increase in IMG applicants or home countries who may retain a greater number of physicians. ${ }^{153}$ This also raises concerns about Western accreditation standards and ultimately the global-local tensions that arise. ${ }^{154}$ Could a move to meet these international accreditation standards create a mismatch with local needs? This is of particular concern where there are significant differences in clinical practice or the type of technology available. In addition, it is challenging to demonstrate that international accreditation confers higher quality patient care in the United States nor in their original country of training. ${ }^{153}$

A global approach to medical education is challenging. The process of consensus and standardization is susceptible to influence by individual conflict of interest, political pressures and differences between how educational activities are reported versus actually delivered in a country. ${ }^{155}$ Our work has elucidated similar concerns with respect to global medical curriculum development, namely political and sociocultural challenges. An additional challenge in global approaches to education is regional variations on what is considered high priority. For example, humanism in medicine is growing in Western contexts. ${ }^{120}$ In other regions moral duties, such as responsibility, receive priority. ${ }^{156}$ The essential consideration of social, cultural and health care system factors ${ }^{86}$ in developing local training content may be addressed in existing global medical curricula.

The main motivation of global medical curricula identified in this scoping review is to promote physician mobility. This reflects the discourse of the internationalization of medicine and medical education. ${ }^{157}$ The internationalization of medicine is a driving factor behind the need for global standards, and physician mobility is a core attribute 
of this. ${ }^{38}$ However, while this may promote individual freedoms, it may lead to increasing maldistribution of health care workers. ${ }^{158}$ The movement of physicians from low-middle income countries creates severe physician shortages and drives the decline in the physician: population ratio. ${ }^{10}$ Mechanisms to accurately monitor and model health human resources globally are challenging, but will be an integral aspect of determining the impact, positive or negative, of promoting greater mobility within the medical workforce.

Global medical education standards, such as those articulated by the World Federation of Medical Education, emphasize certain priorities including a foundation in biomedical sciences which could be a mechanism for perpetuating Western medical priorities. ${ }^{159}$ These priorities may be reflected in the global medical curricula identified in this study, as none referred to traditional medicine or engaging traditional healers in care. The social science priorities recommended focus on professionalism as well as legal and ethical responsibilities. ${ }^{159}$ In an analysis of humanism in global oncology curricula this emphasis on professionalism was also identified. ${ }^{160}$ Despite the importance of traditional medicine in global contexts ${ }^{161,162}$ we were not able to identify content in the existing curricula that addresses traditional medicine, Indigenous health care treatments or their integration into care in global contexts. In addition, access to widely varying technology in different areas of the globe is a point of consideration in the development of global medical curricula. How this is manifest and the degree of impact it has in different specialties is likely variable. Radiation Oncology, a subspecialty field of oncology that is heavily reliant on complex technology, provides an illustrative example. In a global curricula published by the International Atomic Energy Agency (IAEA), they describe 3 skill levels from mandatory (level 1 and 2 ) to desirable (level 3). ${ }^{163}$ These levels reflect varying access to technology. However, one questions if there is a preferable approach to addressing variable access to technology in curriculum design. One may argue curricula should be adapted to suit existing technology, as in the example above, while others may promote education on what is available or possible even if there is no local access. This latter approach may facilitate referral of patients to beneficial resources in other settings, for example the referral of Canadian cancer patients to the United States for Proton therapy ${ }^{164}$ (or may give clinicians a foundation for advocating for new resources for their area. This complex intersection of globalization and technology would benefit from additional study and exploration.

The most common methods used to create the curricula in this study are committees and Delphi processes. These methods are reflective of the recommendations in the literature. ${ }^{1576}$ Without data on the success of implementation and the degree of adoption of these curricula, we cannot provide recommendations as to which methods may produce superior results. However, given the recommendations to engage diverse stakeholders, utilizing mixed methods to achieve this level of engagement may be required. Nine 
percent of the curricula identified in this report applied a mixed methods approach. The limitations of such social consensus processes, such as Delphi methodology and working groups, include a reductionist approach and the tension between a desire for standardization and valuing diversity. ${ }^{85,105,138}$ It is thus important to be mindful that Delphi processes do not methodologically lend themselves to preserving diversity, particularly if the perspectives we hope to incorporate are being made by minority expert voices.

This scoping review has several limitations. We were not able to determine the degree to which an increase in multiregional perspectives in these curricula would result in realworld improvements in local implementation. Answering this question would be better explored through a qualitative approach. Through analysis of the curricular texts alone, we cannot ascertain the degree of engagement from all stakeholders in the development of these curricula. This is an important limitation as it has been stated that failing to engage those who implement the curricula will result in a failure of the curricula to be applied in practice. ${ }^{38}$ To address this concern, future studies should include observations of global curricula development meetings and stakeholder interviews. In addition, all of the authors on this project have a Western background and this may have influenced our interpretation of the data. Our results reflect the West/non-West distinction that is made in the literature we reviewed, however this distinction is likely overly simplistic and future research, in addition to future global curriculum development, should be more sensitive to the diversity within Western and non-Western contexts. We were not able to ascertain a single definition of global or regional with respect to medical curricula in the published literature. As such, with a desire to be comprehensive, we used an inclusive definition of $>1$ country. While this approach fostered inclusivity, an alternate definition may produce a different number of publications. A final consideration, as with all reviews, is that we may not have captured all possible publications on this topic.

\section{Conclusions}

Facilitating physician mobility and improving quality are the main motivations for creating global medical curricula. However, as this review has revealed, the challenges of intercountry variation and the need for a multi-stakeholder approach and implementation need to be considered if we are to address concerns of relevance. These challenges undoubtedly impact uptake of the curricula and can only be addressed by explicit effort to make global curricula applicable to the realities of diverse health care sectors. 


\section{References}

1. World Federation for Medical Education. World Directory of Medical Schools. 2019; https:// wfme.org/world-directory/. Accessed November 27, 2019.

2. Global Health Workforce Alliance. The Institute for International Medical Education. 2019; https://www.who.int/workforcealliance/members_partners/member_list/iime/en/. Accessed November 27, 2019.

3. McMichael AJ. Globalization, climate change, and human health. N Engl J Med. 2013;368(14):1335-1343.

4. Patel S, Jagsi R, Cook N, Hughes-Davies L, Parkinson C. The International Core Literature Consensus (ICLC): an alternative curriculum for Oncologists. J Cancer Educ. 2011;26(3):420-426.

5. Hartzband P, Groopman J. Keeping the patient in the equation—humanism and health care reform. N Engl J Med. 2009;361(6):554-555.

6. Whitehead C. Scientist or science-stuffed? Discourses of science in North American medical education. Med Educ. 2013;47(1):26-32.

7. Frenk J, Chen L, Bhutta ZA, et al. Health professionals for a new century: transforming education to strengthen health systems in an interdependent world. Lancet. 2010;376(9756):19231958.

8. World Federation for Medical Education. About. 2019; https://wfme.org/about-wfme/. Accessed November 27, 2019.

9. Patricio $M$, Harden RM. The Bologna Process-A global vision for the future of medical education. Med Teach. 2010;32(4):305-315.

10. Harden RM. International medical education and future directions: a global perspective. Acad Med. 2006;81(12 Suppl):S22-29.

11. European Union of Medical Specialists. About us: Presentation. 2013; https://www.uems.eu/ about-us/presentation. Accessed November 27, 2019.

12. Spring J. Globalization of Education: An Introduction. 2nd Edition ed. New York: Routledge 2014.

13. Blackmore P, Kandiko CB. Strategic Curriculum Change: Global Trends in Universities 1st Edition ed. London; New York: Routledge 2012.

14. Anderson K, Raza D, Philpott J. Exploring the hidden curriculum of global health. Social Medicine. 2014;8(3):143-146.

15. Bion J, Rothen HU. Models for intensive care training. A European perspective. Am J Respir Crit Care Med. 2014;189(3):256-262.

16. Giuliani M, Frambach J, Broadhurst M, Papadakos J, Fazelad R, Driessen E, Martimianakis M. A Critical Review of Representation in the Development of Global Oncology Curricula and the Influence of Neocolonialism. BMC Med Educ. 2020 Mar 30;20(1):93.

17. Arksey H, O'Malley L. Scoping studies: towards a methodological framework. International Journal of Social Research Methodology. 2005;8(1):19-32. 
18. Martimianakis MA, Michalec B, Lam J, Cartmill C, Taylor JS, Hafferty FW. Humanism, the Hidden Curriculum, and Educational Reform: A Scoping Review and Thematic Analysis. Acad Med. 2015;90(11 Suppl):S5-S13.

19. Abdullah F, Troedsson H, Cherian M. The World Health Organization program for emergency surgical, obstetric, and anesthetic care: from Mongolia to the future. Arch Surg. 2011;146(5):620-623.

20. Adleman J, Gillan C, Caissie A, et al. Development of a Quality and Safety Competency Curriculum for Radiation Oncology Residency: An International Delphi Study. Int J Radiat Oncol Biol Phys. 2017;98(2):428-437.

21. Afolabi B, Olapade-Olaopa EO. International training in medical education - the FAIMER institute 2002 fellowship experience. Afr J Med Med Sci. 2006;35(1):59-67.

22. Ahmed K, Khan R, Mottrie A, et al. Development of a standardised training curriculum for robotic surgery: a consensus statement from an international multidisciplinary group of experts. BJU Int. 2015;116(1):93-101.

23. Ahmed K, Patel S, Aydin A, et al. European Association of Urology Section of Urolithiasis (EULIS) Consensus Statement on Simulation, Training, and Assessment in Urolithiasis. Eur Urol Focus. 2018;4(4):614-620.

24. Alahuhta S, Mellin-Olsen J, Blunnie WP, Knape JT, Section, Board of Anaesthesiology EUoMS. Charter on continuing medical education/continuing professional development approved by the UEMS Specialist Section and European Board of Anaesthesiology. Eur J Anaesthesiol. 2007;24(6):483-485.

25. Almeida MJ. Perspective on South America: the Latin American contribution to the world movement in medical education. Med Educ. 2001;35(8):796-799.

26. Amor B. What competence does a rheumatologist need?: an international perspective. Ann Rheum Dis. 2000;59(8):580-582.

27. European Union of Medical Specialists. Chapter 6, Charter on training of medical specialists in the EU. Requirements for specialty radiotherapy. Radiother Oncol. 2004;70(2):115-116.

28. Antonaci F, Lainez JM, Diener HC, et al. Guidelines for the organization of headache education in Europe: the headache school. Funct Neurol. 2005;20(2):89-93.

29. Arafat R, Askitopoulou H, Della Corte F, Jakubaszko J, Sabbe M. Core curriculum in Emergency Medicine. Eur J Anaesthesiol. 2008;25(8):690-691; author reply 691-692.

30. Are C, Berman RS, Wyld L, Cummings C, Lecoq C, Audisio RA. Global Curriculum in Surgical Oncology. Ann Surg Oncol. 2016;23(6):1782-1795.

31. Are C, Yanala U, Malhotra G, et al. Global Curriculum in Research Literacy for the Surgical Oncologist. Ann Surg Oncol. 2018;25(3):604-616.

32. Artigas A, Pelosi P, Dellweg D, et al. Respiratory critical care HERMES syllabus: defining competencies for respiratory doctors. Eur Respir J. 2012;39(6):1294-1297.

33. Artigas A, Vassilakopoulos T, Brochard L, et al. Respiratory critical care HERMES: a European core syllabus in respiratory critical care medicine. Breathe. 2012;8(3):216-229. 
34. Avgerinos ED, Koupidis SA, Filippou DK. Impact of the European Union enlargement on health professionals and health care systems. Health Policy. 2004;69(3):403-408.

35. Ayu AP, El-Guebaly N, Schellekens A, et al. Core addiction medicine competencies for doctors: An international consultation on training. Subst Abus. 2017;38(4):483-487.

36. Bachmann C, Abramovitch H, Barbu CG, et al. A European consensus on learning objectives for a core communication curriculum in health care professions. Patient Educ Couns. 2013;93(1):18-26.

37. Balch CM, Poston GJ. Training a new generation of surgical oncologists worldwide. Lancet Oncol. 2016;17(6):700-701.

38. Bandaranayake R. The concept and practicability of a core curriculum in basic medical education. Med Teach. 2000;22(6):560-563.

39. Bayram J, Rosborough S, Bartels S, et al. Core curricular elements for fellowship training in international emergency medicine. Acad Emerg Med. 2010;17(7):748-757.

40. Bellou A, Conroy SP, Graham CA. The European curriculum for geriatric emergency medicine. Eur J Emerg Med. 2016;23(4):239.

41. Berberat PO, de Wit NJ, Bockhorn M, Lundell L, Drenth JP. Training innovations in gastroenterology and educational resources: a new vision of gastrointestinal education across Europe. Eur J Gastroenterol Hepatol. 2010;22(12):1393-1396.

42. Berman AC. Anatomy of curriculum: digging to the core. Anat Sci Educ. 2014;7(4):326-328.

43. Besso J, Bhagwanjee S, Takezawa J, Prayag S, Moreno R. A global view of education and training in critical care medicine. Crit Care Clin. 2006;22(3):539-546, x-xi.

44. CoBaTrICE Collaboration. International standards for programmes of training in intensive care medicine in Europe. Intensive Care Med. 2011;37(3):385-393.

45. Bode CO, Nwawolo CC, Giwa-Osagie OF. Surgical education at the West African College of Surgeons. World J Surg. 2008;32(10):2162-2166.

46. Bolon B, Barale-Thomas E, Bradley A, et al. International recommendations for training future toxicologic pathologists participating in regulatory-type, nonclinical toxicity studies. J Toxicol Pathol. 2010;23(3):171-181.

47. BreipohI W, Johansson C, Hansis M, et al. Undergraduate medical education: tendencies and requirements in a rapidly developing Europe. Folia Med (Plovdiv). 2000;42(2):5-16.

48. Brinkman DJ, Tichelaar J, Schutte T, et al. Essential competencies in prescribing: A first european cross-sectional study among 895 final-year medical students. Clin Pharmacol Ther. 2017;101(2):281-289.

49. Brunelli A, Falcoz PE, D'Amico T, et al. European guidelines on structure and qualification of general thoracic surgery. Eur J Cardiothorac Surg. 2014;45(5):779-786.

50. Bundred P, Gibbs T. Medical migration and Africa: an unwanted legacy of educational change. Med Teach. 2007;29(9):893-896.

51. Burkhardt JK, Zinn PO, Bozinov O, Colen RR, Bertalanffy H, Kasper EM. Neurosurgical education in Europe and the United States of America. Neurosurg Rev. 2010;33(4):409-417. 
52. Buth J, Harris PL, Maurer PC, Nachbur B, van Urk H. Harmonization of vascular surgical training in Europe. A task for the European Board of Vascular Surgery (EBVS). Cardiovasc Surg. 2000;8(2):98-103.

53. Carracedo A, Giardina E, Mosquera-Miguel A, Manzo L, Alvarez-Iglesias V, Schneider PM. Making progress in education: The EUROFORGEN master degree pilot project in forensic genetics. Forensic Sci Int Genet. 2017;28:e12-e13.

54. Chan MK, de Camps Meschino D, Dath $D$, et al. Collaborating internationally on physician leadership development: why now? Leadersh Health Serv (Bradf Engl). 2016;29(3):231-239.

55. Chuang LT, Randall TC, Denny L, et al. Sister Society Meeting on Global Education Development and Collaboration: Meeting Report. Int J Gynecol Cancer. 2016;26(6):1186-1188.

56. Ciottone GR, Old A, Nicholas S, Anderson PD. Implementation of an emergency and disaster medical response training network in the Commonwealth of Independent States. J Emerg Med. 2005;29(2):221-229.

57. Cohen H, Saenz R, de Almeida Troncon LE, Lizarzabal M, Olano C. Gastroenterology training in Latin America. World J Gastroenterol. 2011;17(18):2283-2287.

58. Copeland C, Fisher J, Teodorczuk A. Development of an international undergraduate curriculum for delirium using a modified delphi process. Age Ageing. 2018;47(1):131-137.

59. Core Committee Institute for International Medical Education. Global minimum essential requirements in medical education. Med Teach. 2002;24(2):130-135.

60. Costa A, Van Hemelryck F, Aparicio A, et al. Continuing medical education in Europe: towards a harmonised system. Eur J Cancer. 2010;46(13):2340-2343.

61. Cumming A, Ross M. The Tuning Project for Medicine-learning outcomes for undergraduate medical education in Europe. Med Teach. 2007;29(7):636-641.

62. Cuocolo A, Milcinski M, Bischof Delaloy A, European Board of Nuclear M. Syllabus for postgraduate specialisation in Nuclear Medicine. 2006/07 update. Eur J Nucl Med Mol Imaging. 2009;36(3):551-554.

63. Da Silva JA, Faarvang KL, Bandilla K, Woolf AD, Section U, Board of R. UEMS charter on the training of rheumatologists in Europe. Ann Rheum Dis. 2008;67(4):555-558.

64. Danziger-Isakov L, Allen U, Englund J, et al. Recommended Curriculum for Training in Pediatric Transplant Infectious Diseases. J Pediatric Infect Dis Soc. 2015;4(1):4-10.

65. De Korvin G, Delarque A. Physical and rehabilitation medicine section and board of the European Union of Medical Specialists. Community context; history of European medical organizations; actions under way. Ann Phys Rehabil Med. 2009;52(7-8):594-607.

66. De Lange $\mathrm{S}$, Van Aken H, Burchardi H, European Society of Intensive Care M, Multidisciplinary Joint Committee of Intensive Care Medicine of the European Union of Medical S. European Society of Intensive Care Medicine statement: intensive care medicine in Europe-structure, organisation and training guidelines of the Multidisciplinary Joint Committee of Intensive Care Medicine (MJCICM) of the European Union of Medical Specialists (UEMS). Intensive Care Med. 2002;28(11):1505-1511. 
67. De Robertis E, McAdoo J, Pagni R, Knape JT. Core curriculum in emergency medicine integrated in the specialty of anaesthesiology. Eur J Anaesthesiol. 2007;24(12):987-990.

68. Dequeker J, Rasker JJ, Woolf AD. Educational issues in rheumatology. Baillieres Best Pract Res Clin Rheumatol. 2000;14(4):715-729.

69. Dickenson DL, Parker MJ. The European Biomedical Ethics Practitioner Education Project: an experiential approach to philosophy and ethics in health care education. Med Health Care Philos. 1999;2(3):231-237.

70. Dittrich C, Kosty M, Jezdic S, et al. ESMO / ASCO Recommendations for a Global Curriculum in Medical Oncology Edition 2016. ESMO Open. 2016;1(5):e000097.

71. Dittrich C, Stiefel F, Kiss A, Dizon DS. Reply to the letter to the editor 'Integrating communication as a core skill in the global curriculum for medical oncology' by Horlait et al. Ann Oncol. 2017;28(4):905-906.

72. Dubowitz G, Evans FM. Developing a curriculum for anaesthesia training in low- and middle-income countries. Best Pract Res Clin Anaesthesiol. 2012;26(1):17-21.

73. Elliott CG, Barst RJ, Seeger W, et al. Worldwide physician education and training in pulmonary hypertension: pulmonary vascular disease: the global perspective. Chest. 2010;137(6 Suppl):85S-94S.

74. Faarvang KL, da Silva JA. Competencies in rheumatology: a European framework. Best Pract Res Clin Rheumatol. 2009;23(2):145-160.

75. Fahrer R, Jorge MR, Ruiz P. Addressing psychiatric education in Latin America: Challenges and opportunities. International Review of Psychiatry. 2010;22(4):378-381.

76. Fitridge R, Quigley F, Vicaretti M. Should we develop a core international curriculum for Vascular and Endovascular Surgery? Eur J Vasc Endovasc Surg. 2010;39 Suppl 1:S10-14.

77. Francis N, Penna M, Mackenzie H, Carter F, Hompes R, International Ta TMEECG. Consensus on structured training curriculum for transanal total mesorectal excision (TaTME). Surg Endosc. 2017;31(7):2711-2719.

78. Gappa M, Paton J, Baraldi E, et al. Paediatric HERMES: update of the European Training Syllabus for Paediatric Respiratory Medicine. Eur Respir J. 2009;33(3):464-465.

79. Garcia de Leonardo C, Ruiz-Moral R, Caballero F, et al. A Latin American, Portuguese and Spanish consensus on a core communication curriculum for undergraduate medical education. BMC Med Educ. 2016;16:99.

80. Garofalo M, Aggarwal R. Competency-Based Medical Education and Assessment of Training: Review of Selected National Obstetrics and Gynaecology Curricula. J Obstet Gynaecol Can. 2017;39(7):534-544 e531.

81. Gimelli A, Neglia D, Schindler TH, Cosyns B, Lancellotti P, Kitsiou A. Nuclear cardiology core syllabus of the European Association of Cardiovascular Imaging (EACVI). Eur Heart J CardiovasC Imaging. 2015;16(4):349-350.

82. Giuliani ME, Gillan C, Milne RA, Uchino M, Millar BA, Catton P. Determining an imaging literacy curriculum for radiation oncologists: an international Delphi study. Int J Radiat Oncol Biol Phys. 2014;88(4):961-966. 
83. Goligher EC, Ferguson ND, Kenny LP. Core competency in mechanical ventilation: development of educational objectives using the Delphi technique. Crit Care Med. 2012;40(10):28282832.

84. Gordon M, Baker P, Catchpole K, Darbyshire D, Schocken D. Devising a consensus definition and framework for non-technical skills in healthcare to support educational design: A modified Delphi study. Med Teach. 2015;37(6):572-577.

85. Gukas ID. Global paradigm shift in medical education: issues of concern for Africa. Med Teach. 2007;29(9):887-892.

86. Hamdy H. The Problem and Goals Are Global, the Solutions Are Local: Revisiting Quality Measurements and the Role of the Private Sector in Global Health Professions Education. Acad Med. 2017;92(8):1082-1085.

87. Hansen $\mathrm{HH}$, Bajorin DF, Muss HB, et al. Recommendations for a Global Core Curriculum in Medical Oncology. Ann Oncol. 2004;15(11):1603-1612.

88. Heidbuchel H, Papadakis M, Panhuyzen-Goedkoop N, et al. Position paper: proposal for a core curriculum for a European Sports Cardiology qualification. Eur J Prev Cardiol. 2013;20(5):889903.

89. Heitkamp DE, Johnson KS, Suh RD, et al. Sustaining Change in Radiology Education: The Need for Universal Curricula. J Am Coll Radiol. 2017;14(6):804-807.

90. Hobgood C, Anantharaman V, Bandiera G, et al. International Federation for Emergency Medicine Model Curriculum for Emergency Medicine Specialists. Emerg Med Australas. 2011;23(5):541-553.

91. Hodges BD, Maniate JM, Martimianakis MA, Alsuwaidan M, Segouin C. Cracks and crevices: globalization discourse and medical education. Med Teach. 2009;31(10):910-917.

92. International Federation of Medical Students A, European Medical Students A, Hilgers J, De Roos P. European core curriculum-the students' perspective, Bristol, UK, 10 July 2006. Med Teach. 2007;29(2-3):270-275.

93. Kelly AM, Mullan PB. Designing a Curriculum for Professionalism and Ethics Within Radiology: Identifying Challenges and Expectations. Acad Radiol. 2018;25(5):610-618.

94. Khanam NN, Chowdhury AA. Globalization of Medical Education Curriculum. Bangladesh Journal of Obstetrics \& Gynaecology. 2015;30(1):37-42.

95. Lalloo D, Demou E, Kiran S, Cloeren M, Mendes R, Macdonald EB. International perspective on common core competencies for occupational physicians: a modified Delphi study. Occup Environ Med. 2016;73(7):452-458.

96. Lappin DW, Cannata-Andia JB, Nephrology USi. Updated programme for harmonization of training in nephrology in the European Union. Clin Kidney J. 2013;6(1):116-121.

97. Lee AG, Golnik KC, Tso MO, Spivey B, Miller K, Gauthier TM. The international council of ophthalmology: vision for ophthalmic education in an interdependent world. Am J Ophthalmol. 2012;154(4):620-624 e622.

98. Leinster S. Standards in medical education in the European Union. Med Teach. 2003;25(5):507509. 
99. Leslie K. The globalization of training in adolescent health and medicine: one size does not fit all. International Journal of Adolescent Medicine and Health. 2015;28(3):279-283.

100. Macerollo A, Struhal W, Sellner J. Harmonization of European neurology education: the junior doctor's perspective. Neurology. 2013;81(18):1626-1629.

101. Makoul G, Schofield T. Communication teaching and assessment in medical education: an international consensus statement. Netherlands Institute of Primary Health Care. Patient Educ Couns. 1999;37(2):191-195.

102. Masud T, Blundell A, Gordon AL, et al. European undergraduate curriculum in geriatric medicine developed using an international modified Delphi technique. Age Ageing. 2014;43(5):695702.

103. Mayberry JF. The development of medical education in Eastern Europe during the 20th century and the emergence of 'English parallel' courses. Scott Med J. 2013;58(1):46-52.

104. McKendrick MW, European Union of Medical S. The European Union of Medical Specialties core training curriculum in infectious diseases: overview of national systems and distribution of specialists. Clin Microbiol Infect. 2005;11 Suppl 1:28-32.

105. McLintock C, Pabinger I, Bauer KA, et al. International Society on Thrombosis and Haemostasis core curriculum project: core competencies in clinical thrombosis and hemostasis. J Thromb Haemost. 2016;14(1):3-27.

106. McLintock C, Ross M. International Society on Thrombosis and Haemostasis core curriculum project: core competencies in clinical thrombosis and hemostasis: reply. Journal of Thrombosis and Haemostasis. 2016;14(6):1318-1319.

107. Meert A-P, Noël J-L, Boffetta P, et al. Thoracic Oncology HERMES: a European syllabus towards a harmonised education and training of Thoracic Oncology specialists. Breathe. 2013;9(5):381392.

108. Mitchell S, Rohde G. Introducing an updated international ERS syllabus for postgraduate training 2017Setting standards for training and certification in respiratory medicine. Breathe (Sheff). 2017;13(3):e53-e55.

109. Moxham B, McHanwell S, Plaisant O, Pais D. A core syllabus for the teaching of neuroanatomy to medical students. Clinical Anatomy. 2015;28(6):706-716.

110. Moxham BJ, Plaisant O, Smith CF, Pawlina W, McHanwell S. An approach toward the development of core syllabuses for the anatomical sciences. Anat Sci Educ. 2014;7(4):302-311.

111. Naredi P, Audisio RA, Taylor I. Why do we need a core curriculum in surgical oncology in Europe? Surg Oncol. 2008;17(4):267-269.

112. Nieman K, Achenbach S, Pugliese F, Cosyns B, Lancellotti P, Kitsiou A. Cardiac computed tomography core syllabus of the European Association of Cardiovascular Imaging (EACVI). Eur Heart J Cardiovasc Imaging. 2015;16(4):351-352.

113. Norrby SR, Carbon C. Report of working group 3: specialist training and continuing medical education/professional development in the infection disciplines. Clin Microbiol Infect. 2005;11 Suppl 1:46-49. 
114. Oduntan OA, Mashige KP, Kio FE, Boadi-Kusi SB. Optometric education in Africa: historical perspectives and challenges. Optom Vis Sci. 2014;91(3):359-365.

115. Okel J, Okpechi IG, Qarni B, et al. Nephrology training curriculum and implications for optimal kidney care in the developing world. Clin Nephrol. 2016;86 (2016)(13):110-113.

116. Oktay C. International Federation for Emergency Medicine Model Curriculum for Emergency Medicine Specialists. Turkish Journal of Emergency Medicine. 2011;11(3):91-93.

117. Palange P, Noel JL, Simonds AK. The European Respiratory Society: future directions in medical education. Eur Respir J. 2011;38(3):498-499.

118. Pantano P, Chollet F, Paulson O, et al. EFNS Task Force on Teaching of Neuroimaging in Neurology Curricula in Europe: present status and recommendations for the future. Eur J Neurol. 2001;8(6):541-548.

119. Parrish RK, Tso MOM. Principles and Guidelines of a Curriculum for Ophthalmic Education of Medical Students: Presented by International Task Force on Opthalamic Education of Medical Students-On behalf of the International Council of Opthamology (ICO). Klin Monatsbl Augenheilkd. 2006;223(S 5):S1-S19.

120. Pfeiffer S, Chen Y, Tsai D. Progress integrating medical humanities into medical education: a global overview. Curr Opin Psychiatry. 2016;29(5):298-301.

121. Pfenninger EG, Domres BD, StahI W, Bauer A, Houser CM, Himmelseher S. Medical student disaster medicine education: the development of an educational resource. Int J Emerg Med. 2010;3(1):9-20.

122. Phadke K, Bagga A. Training in pediatric nephrology for developing countries. Pediatr Nephrol. 2005;20(9):1205-1207.

123. Pineda C, Reginato AM, Flores V, et al. Pan-American League of Associations for Rheumatology (PANLAR) recommendations and guidelines for musculoskeletal ultrasound training in the Americas for rheumatologists. J Clin Rheumatol. 2010;16(3):113-118.

124. Prigent A, Huic D, Costa DC. Syllabus for Postgraduate Specialization in Nuclear Medicine-2011/2012 Update: nuclear medicine training in the European Union. Eur J Nucl Med Mol Imaging. 2012;39(4):739-743.

125. Prigent A, Hustinx R, Costa DC, et al. Nuclear medicine training in the European Union: 2015 update. Eur J Nucl Med Mol Imaging. 2016;43(3):583-596.

126. Rehani B, Brown I, Dandekar S, et al. Radiology Education in Africa: Analysis of Results From 13 African Countries. J Am Coll Radiol. 2017;14(2):247-252.

127. Reisman Y, Eardley I, Porst H, Multidisciplinary Joint Committee on Sexual M. New developments in education and training in sexual medicine. J Sex Med. 2013;10(4):918-923.

128. Sachdev P. Core Curriculum in Neuropsychiatry of the International Neuropsychiatric Association*. In: Miyoshi K, Morimura Y, Maeda K, eds. Neuropsychiatric Disorders. Tokyo: Springer Japan; 2010:317-346.

129. Salmon M, Landes M, Hunchak C, et al. Getting It Right the First Time: Defining Regionally Relevant Training Curricula and Provider Core Competencies for Point-of-Care Ultrasound Education on the African Continent. Ann Emerg Med. 2017;69(2):218-226. 
130. San Luis TO, Jr., Vinjamuri S. Challenges of developing a pan-Asian curriculum: lessons for global nuclear medicine training. Nucl Med Commun. 2012;33(11):1119-1121.

131. Schwarz MR, Wojtczak A. Global minimum essential requirements: a road towards competence-oriented medical education. Med Teach. 2002;24(2):125-129.

132. Section and Board of Anaesthesiology European Union of Medical specialists, Carlsson C, Keld $\mathrm{D}$, et al. Education and training in anaesthesia-revised guidelines by the European Board of Anaesthesiology, Reanimation and Intensive Care. Eur J Anaesthesiol. 2008;25(7):528-530.

133. Sefton AJ. New approaches to medical education: an international perspective. Med Princ Pract. 2004;13(5):239-248.

134. Silva H, Bühler F, Maillet B, et al. Continuing Medical Education and Professional Development in the European Union. Pharmaceutical Medicine. 2012;26.

135. Silva $\mathrm{H}$, Stonier $\mathrm{P}$, Buhler $\mathrm{F}$, et al. Core competencies for pharmaceutical physicians and drug development scientists. Front Pharmacol. 2013;4:105.

136. Simpson P. Training, assessment and accreditation in anaesthesiology and the implications for the European Union. Eur J Anaesthesiol. 2003;20(9):679-681.

137. Singh P, Aggarwal R, Zevin B, Grantcharov T, Darzi A. A Global Delphi Consensus Study on Defining and Measuring Quality in Surgical Training. Journal of the American College of Surgeons. 2014;219(3):346-353.e347.

138. Skeith L, Rodger MA, Lee AY, Kahn SR, Bates SM, Gonsalves C. International Society on Thrombosis and Haemostasis core curriculum project: core competencies in clinical thrombosis and hemostasis: comment. J Thromb Haemost. 2016;14(6):1316-1317.

139. Skirton H, Lewis C, Kent A, Coviello DA, Members of Eurogentest U, Committee EE. Genetic education and the challenge of genomic medicine: development of core competences to support preparation of health professionals in Europe. Eur J Hum Genet. 2010;18(9):972-977.

140. Soldatos C, Mavreas V, Kaprinis G, et al. Inter-university harmonization of undergraduate and graduate education in psychiatry. Consensus statement of the Hellenic College of Academic Psychiatry. Archives of Hellenic Medicine. 2006;23(4):379-392.

141. Spiwak R, Lett R, Rwanyuma L, Logsetty S. Creation of a standardized burn course for Low Income Countries: meeting local needs. Burns. 2014;40(7):1292-1299.

142. Struhal W, Mellgren SI, Grisold W. Three important steps to European neurology harmonization: core curriculum, visitation program, European board examination. Eur J Neurol. 2013;20(8):e101-104.

143. Tabin N, Mitchell S, O'Connell E, Stolz D, Rohde G. Update of the ERS international Adult Respiratory Medicine syllabus for postgraduate training. Breathe (Sheff). 2018;14(1):19-28.

144. Tandeter H, Carelli F, Timonen M, et al. A 'minimal core curriculum' for Family Medicine in undergraduate medical education: a European Delphi survey among EURACT representatives. Eur J Gen Pract. 2011;17(4):217-220.

145. Tolba RH, Czigany Z, Osorio Lujan S, et al. Defining Standards in Experimental Microsurgical Training: Recommendations of the European Society for Surgical Research (ESSR) and the International Society for Experimental Microsurgery (ISEM). Eur Surg Res. 2017;58(5-6):246-262. 
146. Totsch M, Cuvelier C, Vass L, Fassina A, Paris USBoPmi. The UEMS section/board of pathology, chapter 6: requirement for recognition of postgraduate training in pathology: a presentation of the paris document. Cytopathology. 2012;23(5):295-299.

147. Tubbs RS, Sorenson EP, Sharma A, et al. The development of a core syllabus for the teaching of head and neck anatomy to medical students. Clin Anat. 2014;27(3):321-330.

148. Turner S, Seel M, Trotter T, et al. Defining a Leader Role curriculum for radiation oncology: A global Delphi consensus study. Radiother Oncol. 2017;123(2):331-336.

149. Van Gessel EF, Ostergard HT, Niemi-Murola L. Harmonisation of anaesthesiology training in Europe. Best Pract Res Clin Anaesthesiol. 2012;26(1):55-67.

150. Woolf A. Specialist training in rheumatology in Europe. Rheumatology (Oxford, England). 2002;41:1062-1066.

151. CoBaTrICE Collaboration, Bion JF, Barrett H. Development of core competencies for an international training programme in intensive care medicine. Intensive Care Med. 2006;32(9):13711383.

152. Bandaranayake RC. Implementing change in medical education in developing countries. Med Teach. 1989;11(1):39-45.

153. Tackett S. Examining the Educational Commission for Foreign Medical Graduates Announcement Requiring Medical School Accreditation Beginning in 2023. Acad Med. 2019;94(7):943949.

154. Ho MJ, Abbas J, Ahn D, Lai CW, Nara N, Shaw K. The "Glocalization" of Medical School Accreditation: Case Studies From Taiwan, South Korea, and Japan. Acad Med. 2017;92(12):1715-1722.

155. Lindgren S, Karle H. Social accountability of medical education: aspects on global accreditation. Med Teach. 2011;33(8):667-672.

156. Kim S, Choi S. The Medical Professionalism of Korean Physicians: Present and Future. BMC Med Ethics. 2015;16:56.

157. Harden RM, Davis MH. AMEE Medical Education Guide No. 5. The core curriculum with options or special study modules. Medical Teacher. 1995;17(2):125-148.

158. Salafsky B, Glasser M, Ha J. Addressing issues of maldistribution of health care workers. Ann Acad Med Singapore. 2005;34(8):520-526.

159. Maccarrick G. A practical guide to using the World Federation for Medical Education standards. WFME 2: educational program. Ir J Med Sci. 2010;179(4):489-491.

160. Giuliani M, Martimianakis M, Broadhurst M, Papadakos J, Driessen E, Frambach J. Humanism in Global Oncology Curricula: An Emerging Priority. 2019.

161. World Health Organization. WHO Traditional Medicine Strategy: 2014-2023. Hong Kong SAR, China2013: http://apps.who.int/medicinedocs/documents/s21201en/s21201en.pdf. Accessed November 27, 2019

162. Gall A, Leske S, Adams J, et al. Traditional and Complementary Medicine Use Among Indigenous Cancer Patients in Australia, Canada, New Zealand, and the United States: A Systematic Review. Integr Cancer Ther. 2018;17(3):568-581. 
163. International Atomic Energy Agency. IAEA Syllabus for the Education and Training of Radiation Oncologists. Endorsed by the American Society for Radiation Oncology (ASTRO) and the European Society for Therapeutic Radiology and Oncology (ESTRO). Vienna2009.

164. Tsang DS, Patel S. Proton beam therapy for cancer. CMAJ. 2019;191(24):E664-E666.

\section{Supplemental File 1: Ovid MEDLINE Search Strategy for Motivation and Challenges in the Development of Global Medical Curricula: A scoping review}

\begin{tabular}{|c|c|c|c|c|}
\hline \multicolumn{5}{|c|}{ Ovid MEDLINE(R) 1998 to March Week 42018} \\
\hline $\begin{array}{c}\text { Medical component } \\
\text { including MeSH/ keywords } \\
\text { combined with "OR" }\end{array}$ & AND & $\begin{array}{l}\text { Curriculum component } \\
\text { including MeSH/keywords } \\
\text { combined with "OR" }\end{array}$ & AND & $\begin{array}{c}\text { Global component } \\
\text { including MeSH/keywords } \\
\text { combined with "OR" }\end{array}$ \\
\hline $\begin{array}{l}\text { Medicine/ } \\
\text { exp Physicians/ } \\
\text { exp Medical Oncology/ } \\
\text { exp Academic Medical Centers/ } \\
\text { exp Societies, Medical/ } \\
\text { Hospital Medicine/ } \\
\text { exp Hospitals, Teaching/ } \\
\text { Faculty, Medical/ } \\
\text { exp Education, Medical/ } \\
\text { Medical Students/ } \\
\text { exp “Internship and Residency"/ } \\
\text { Clinical Clerkship/oncologist. } \\
\text { mp,kw. } \\
\text { (academ* adj2 (medicine? or } \\
\text { medical?)).mp,kw. } \\
\text { ((medical or surgical) adj2 so- } \\
\text { ciet*).mp,kw. } \\
\text { (hospital? adj2 (medicine? or } \\
\text { teaching)).mp,kw. } \\
\text { (facult* adj2 (medicine? or medi- } \\
\text { cal?)).mp,kw. } \\
\text { (educat* adj2 (medicine? or medi- } \\
\text { cal?)).mp,kw. } \\
\text { (student? adj2 (medicine? or } \\
\text { medical?)).mp,kw. } \\
\text { (graduat* adj2 (medicine? or } \\
\text { medical?)).mp,kw. } \\
\text { (postgraduat* adj2 (medicine? or } \\
\text { medical?)).mp,kw. } \\
\text { residen*.tw,kw. } \\
\text { internship*.mp,kw. } \\
\text { intern?.mp,kw. } \\
\text { fellowship*.mp,kw. } \\
\text { fellow?.mp,kw. } \\
\text { clerkship*.mp,kw. } \\
\text { preclerkship*.mp,kw. }\end{array}$ & & $\begin{array}{l}\text { exp Curriculum/ } \\
\text { Educational Measurement/ } \\
\text { Models, Educational/ } \\
\text { Program Development/ } \\
\text { Program Evaluation/ } \\
\text { Training Support/ } \\
\text { curricul*.mp,kw. } \\
\text { (educat* adj2 measur*).mp,kw. } \\
\text { (educat* adj2 model*).mp,kw. } \\
\text { (program* adj2 develop*). } \\
\text { mp,kw. } \\
\text { (program* adj2 evaluat*). } \\
\text { mp,kw. } \\
\text { (train* adj2 program*).mp,kw. } \\
\text { (requir* adj2 program*).mp,kw. } \\
\text { (core? adj2 program*).mp,kw. } \\
\text { (learn* adj2 program*).mp,kw. } \\
\text { (teach* adj2 program*).mp,kw. } \\
\text { (standard* adj2 program*). } \\
\text { mp,kw. } \\
\text { (core? adj2 subject?).mp,kw. } \\
\text { (requir* adj2 subject?).mp,kw. } \\
\text { (standard* adj2 subject?). } \\
\text { mp,kw. } \\
\text { (core? adj2 course?).mp,kw. } \\
\text { (requir* adj2 course?).mp,kw. } \\
\text { (standard* adj2 course?). } \\
\text { mp,kw. } \\
\text { pedagog*.mp,kw. } \\
\text { outline?.tw,kw. } \\
\text { syllab*.tw,kw. } \\
\text { module?.tw,kw. }\end{array}$ & & $\begin{array}{l}\text { Global Health/ } \\
\text { exp Internationality/ } \\
\text { exp International Coope- } \\
\text { ration/ } \\
\text { International Agencies/ } \\
\text { international*.mp,kw. } \\
\text { global*.tw,kw. } \\
\text { intercontinental*.tw,kw. } \\
\text { inter-continental*.tw,kw. } \\
\text { universal*.tw,kw. } \\
\text { multinational*.tw,kw. } \\
\text { multi-national*.tw,kw. } \\
\text { world wide*.tw,kw. } \\
\text { worldwide**.tw,kw. } \\
\text { "without border?".mp,kw. } \\
\text { imperiali?m.mp,kw. } \\
\text { coloniali?m.mp,kw. } \\
\text { anti-colonial*.mp,kw. } \\
\text { anticolonial*.mp,kw. } \\
\text { neocolonial*.mp,kw. } \\
\text { neo-colonial*.mp,kw. } \\
\text { postcolonial*.mp,kw. } \\
\text { post-colonial*.mp,kw }\end{array}$ \\
\hline
\end{tabular}

The search has been conducted in the following databases: Embase, Epub Ahead of Print, Cochrane CT, Cochrane SR, Psyclnfo, CINAHL, ERIC, Scopus, African Index Medicus (AIM), and LILACS (Latin America). 
CHAPTER 3

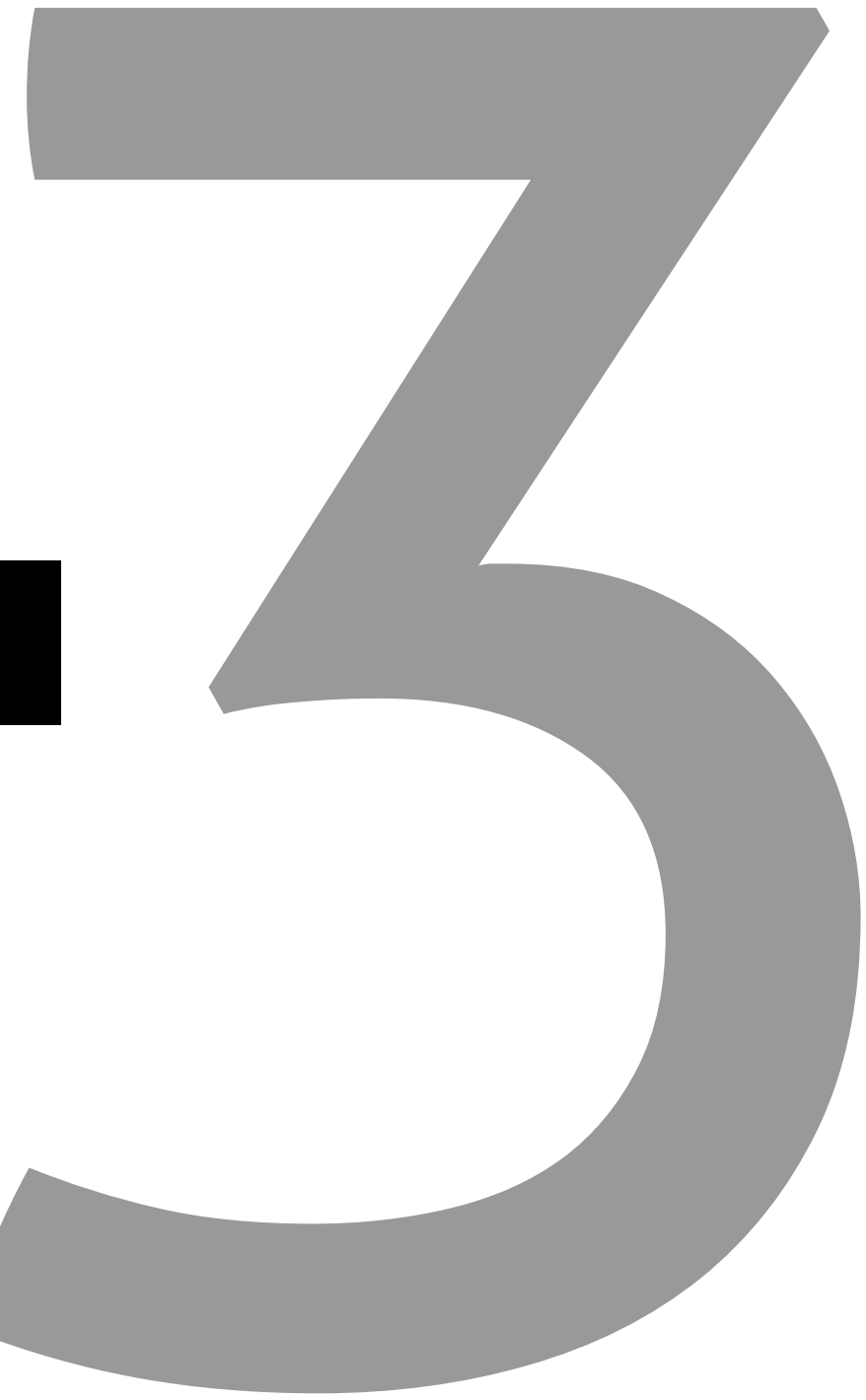




\section{A Critical Review of Representation in the Development of Global Oncology Curricula and the Influence of Neocolonialism}

Published as: Giuliani M, Frambach J, Broadhurst M, Papadakos $J$, Fazelad R, Driessen E, Martimianakis M. A Critical Review of Representation in the Development of Global Oncology Curricula and the Influence of Neocolonialism. BMC Med Educ. 2020 Mar 30;20(1):93. 


\section{Background:}

Global curricular homogenization is purported to have a multitude of benefits. However, homogenization, as typically practiced has been found to promote largely Western ideals. The purpose of this study was to explore the issue of representation in the development of global oncology curricula.

\section{Methods:}

This systematic review of global oncology curricula involved a comprehensive search strategy of eight databases from inception to December 2018. Where available, both controlled vocabulary terms and text words were used. Two investigators independently reviewed the publications for eligibility. Full global/core oncology curricular documents were included. Data analysis included exploration of representation across a number of axes of power including sex and geographic sector, consistent with a neocolonial approach.

\section{Results:}

32,835 documents were identified in the search and 17 remained following application of the inclusion/exclusion criteria. Eleven of 17 papers were published from 2010-2018 and 13 curricula originated from Europe. The 17 curricula had 300 authors; 207 were male and most were from Europe $(n=190 ; 64 \%)$ or North America ( $n=73 ; 24 \%)$. The most common curricular purposes were promoting quality patient care $(n=11)$, harmonization of training standards $(n=10)$, and facilitating physician mobility $(n=3)$. The methods for creation of these curricula were most commonly a committee or task force $(n=10)$. Over time there was an increase in the proportion of female authors and the number of countries represented in the authorship.

\section{Conclusion:}

Existing global oncology curricula are heavily influenced by Western male authors and as a result may not incorporate relevant socio-cultural perspectives impacting care in diverse geographic settings. 


\section{Background}

In cancer education the profound mismatch between the training curricula for healthcare professionals and the needs of patients, families and the health-care system, such as team training to foster shared care models, is argued to fuel a healthcare crisis ${ }^{1}$. The sources of these mismatches are not fully understood, however, the potential overarching influence of Western medical priorities, in the form of neocolonialism, might be a contributing factor. An example of this includes the emphasis on the biomedical model of healthcare ${ }^{2}$ at the exclusion of other ways of orienting to care and illness. This perpetuation of a Western biomedical model has also been identified by Fouad, in global oncology work ${ }^{3}$. This deserves further study as global, core or regional oncology curricula have been published by several international agencies ${ }^{4.8}$ and improved understanding of how representation of non-Western values and approaches may inform the development of future global focused curricula or the use of existing curricula in diverse parts of the world.

The homogenization of curricula through the spread of Western ideals is purported to have a multitude of benefits ${ }^{9}$ including the recognition of credentials globally and improving the quality of education and ultimately patient care. Medicine is an example of a'credential society' and in situations where credentials are not universally recognized there is potential for brain waste ${ }^{10}$. Brain waste is a situation where migrant workers are not able to obtain employment commensurate to their educational qualifications ${ }^{9}$ and can be related to brain drain (the migration of workers from low-middle income to high income countries). Brain waste is of significant concern to migrating physicians whereupon, with their arrival in high-income countries, they cannot practice as physicians ${ }^{11}$. In situations where there is great global need for health professional services, such as the growing health professional crisis in oncology, opportunities to minimize brain waste are encouraged ${ }^{12}$. Efforts, including standardization or training and global certification, which may ultimately reduce brain waste, may disproportionately benefit high-income countries. However, notwithstanding the purported benefits of standardizing health education training, the imposition of Western ideals across the world perpetuates neocolonial relationships, which may in turn lead to a mismatch between global priorities and local needs ${ }^{13}$. The increasing brain circulation experienced with the movement of cancer professionals to and from their home countries or regions, the mobility of patients seeking care and the introduction of international accrediting bodies ${ }^{14}$ has resulted in the development of new global/core curricula. This creates an urgency to explore the influence of neocolonialism in global oncology curricula to inform future curricular development efforts that are sensitive to the needs of diverse regions of the world. A neocolonial approach allows for an in-depth analysis of socio-cultural and political imbalances perpetuated through the circulation of knowledge and educational tools. Table 1 outlines key concepts used in this paper. Our work is an effort to start such an analysis in the field of global oncology 
by exploring as a first step representation, a core tenet of neocolonial analysis, in the development of global oncology curricula.

Table 1: Theoretical Framework Terminology

\begin{tabular}{|c|c|}
\hline Term & Explanation \\
\hline $\begin{array}{l}\text { The West and Western } \\
\text { perspective }\end{array}$ & $\begin{array}{l}\text { This is the perspective of countries whose knowledge and traditions is strongly } \\
\text { linked to European immigration including Oceania and the Americas. }\end{array}$ \\
\hline Intersections of power & $\begin{array}{l}\text { This framework articulates how different power systems such as, but not limited } \\
\text { to, class, race and gender interact and how different groups are impacted by these } \\
\text { power systems [39]. }\end{array}$ \\
\hline Neocolonialism & $\begin{array}{l}\text { Neocolonialism describes a form of imperialism which is associated with glo- } \\
\text { bal capitalism and activities of Western media(44). Neocolonialism references a } \\
\text { Western dominated reform agenda and has implications for medical education in } \\
\text { non-Western health care contexts }(30,44) \text {. }\end{array}$ \\
\hline Anti-colonial & $\begin{array}{l}\text { Anti-colonial perspective begins from the standpoint of marginalized peoples, } \\
\text { perspectives or knowledge. The main goal is to provide a different view-point on } \\
\text { dominant perspectives (2). }\end{array}$ \\
\hline $\begin{array}{l}\text { Global North and } \\
\text { Global South }\end{array}$ & $\begin{array}{l}\text { The Global North and Global South dichotomy reflects both a political and soci- } \\
\text { oeconomic divide. The Global North includes Europe, Canada, United States and } \\
\text { some of Asia (Hong Kong, Singapore, South Korea and Taiwan). The Global South } \\
\text { consists of Latin America, Africa, the Middle East, the remainder of Asia and the } \\
\text { Caribbean countries. }\end{array}$ \\
\hline Post-colonial & $\begin{array}{l}\text { Postcolonial theory explores the implications of colonial practices. It is often opera- } \\
\text { tionalized by exploring power relationships (45). }\end{array}$ \\
\hline $\begin{array}{l}\text { Socio-cultural } \\
\text { Representation }\end{array}$ & $\begin{array}{l}\text { Collective elaborations of social determinants of health specific to a region and } \\
\text { cultural dimensions present in this curricular process by individual members. }\end{array}$ \\
\hline $\begin{array}{l}\text { Geographic } \\
\text { Representation }\end{array}$ & $\begin{array}{l}\text { Country or regional perspectives present in this curricular process by individual } \\
\text { members. }\end{array}$ \\
\hline
\end{tabular}

The Best Evidence in Medical Education movement aims to promote a strong evidence base in a variety of topics on medical education ${ }^{15}$ As a result, medical curricula are expected to be grounded and developed through educational research principles. Ad hoc teaching practices are questioned and those founded in educational research are given pre-eminence ${ }^{16}$. As the majority of the research in medical education is currently published in English language journals with origins in European and North American publishing houses ${ }^{3}$ one must question if these Western priorities may dominate curriculum development and dissemination efforts. Arguably, in an effort to promote an evidence base in medical education, traditional beliefs and practices are overlooked while justifying the use of western pedagogical practices and priorities in non-western parts of the world ${ }^{16}$ In other words, a challenge in establishing 'core' curricula in the current medical education landscape is the tension between meeting local needs and achieving international standards ${ }^{16}$. This can be particularly difficult for humanistic competencies such as professionalism ${ }^{17}$. The resistance to the inclusion of new concepts and content in curriculum redesign efforts is well recognized ${ }^{16}$. It is this tension between the need for reform and the desire to maintain some existing practices that make the exploration of representation in the development of global curricula an important area for study. 
We asked, is there a mechanism currently to engage diverse international players in the development of global oncology curricula ${ }^{18}$ ?

The purpose of this study was to explore the issue of representation in global oncology curricula by determining what global curricula exist for oncology, who has developed them, for what purpose and what methods were used in their development. Using an anti-colonial approach in our analysis we explored whether inequities in perspectives exist in global oncology curriculum development work.

\section{Methods}

\section{Data Collection}

For this systematic review we incorporated an anti-colonial analytical approach with a comprehensive search strategy that included specifically looking for curricula from nonWestern regions. To accomplish this, we included a comprehensive search of the published literature without any language restrictions. We hand searched the reference list of our included publications to ensure no other relevant publications were missed. In addition, we reviewed the websites of oncology organizations globally to look for global oncology curricula. We also included non-medical expert curricular content in our search to include curricula that may not focus on the dominant biomedical model.

We searched for global oncology curricula in the following databases from inception to December 2018; Medline, EMBASE, Cochrane Central Register of Controlled Trials, Cochrane Database of Systematic Reviews, Ovid MEDLINE ${ }^{\circledR}$ Epub Ahead of Print and In-Process \& Other Non-Indexed Citations, PsycINFO, all from the OvidSP platform; and CINAHL from EBSCOhost. There were no language or date restrictions because we wanted to ensure we included publications from all regions. Where available, both controlled vocabulary terms and text words were used to maximize our search results and account for global linguistic variations in the subject components for oncology curriculum/education, global and humanistic. We included a variety of terms to ensure we captured all manner of curricula that focused on oncology training including supportive care and non-medical expert knowledge domains and skills to ensure we captured diverse intersections of power. See Supplementary File 1.

In addition, a hand search of major international cancer organizations including The American Society of Clinical Oncology $(\mathrm{ASCO})^{19}$, the American Society for Radiation Oncology (ASTRO) ${ }^{20}$, The European Society for Radiotherapy and Oncology (ESTRO) ${ }^{21}$, the African Organization for Research and Training in Cancer (AORTIC) ${ }^{22}$, The Royal Australian and New Zealand College of Radiologists (RANZCR) ${ }^{23}$, The Federation of Asian Organizations for Radiation Oncology (FARO) ${ }^{24}$, The Asociacion Ibero Latinoamericana de 
Terapia Radiante Oncologica (ALATRO)25, The Canadian Association of Radiation Oncology $(\mathrm{CARO})^{26}$, The European Society of Medical Oncology (ESMO) ${ }^{27}$ and the International Atomic Energy Agency (IAEA) ${ }^{28}$ was conducted to ensure curricula which were not published were included to mitigate Western publication bias in this review.

Duplicates were removed from the search by the information specialist. Two reviewers independently screened the curricula retrieved from the search. Consensus was reached on decisions to include or exclude potentially eligible curricula, with any disagreements resolved by adjudication by a third reviewer to make the final decision on eligibility for full-text review as necessary. For all eligible curricula identified the full text curricula were retrieved for detailed review, and independently screened by two reviewers. Any disagreements on inclusion of these curricula was resolved through adjudication by a third reviewer. A PRISMA flow chart was used to document the screening process.

The following inclusion and exclusion criteria were used for the systematic review. Curricula were included if their focus was on postgraduate medical education or residency level training in an oncology discipline (medical oncology, radiation oncology, and any surgical oncology specialty) their scope was global or multi-country/regional at a minimum. Regional was defined as the curricula focused on or was developed for use in two or more countries. For the purposes of this study a 'global curriculum' was conceptualized as a text, which intends to use a common vocabulary and shared philosophy, and which describes an outcome, including competency items, that are intended to be applicable across nations. The full curricula must be available either in the publication, as an online supplement or by contacting the authors or sponsoring institution. Papers were excluded if they did not include a curriculum (such as opinion papers, job descriptions, scopes of practice statements, program guidance documents, and position statements etc) because we focused on curricula as they are currently developed and in use. Curricula designed for undergraduate medical education, continuing medical education or nonmedical professions were also excluded as they did not address the question of training for certification in an oncology specialty.

\section{Data Analysis}

As mentioned above, the importance of representation in global health work has been stated by major healthcare agencies including the World Health Organization ${ }^{29}$. Anticolonial theory has been previously used to explore power relationships in global health in medical education, including issues of representation ${ }^{30,31}$. We were specifically interested in issues of representation in knowledge creation activities associated with the construction of global oncology curricula ${ }^{2}$ and thus drew on anti-colonial theory to facilitate our analysis. 
The curricular documents were analyzed and coded using NVivo version $11^{32}$. Demographic details were extracted from the curricula including the medical specialties targeted by the curricula, the publication year, the number of authors, the authors' sex, the authors medical specialty, the authors' country, and data on translation from the primary language of publication to other languages. The purpose of the curricula and the methods used to develop the curricula were identified and coded. This analysis included application of an anti-colonial frame to determine representation across a number of axes of power including sex, language, profession and geographic sector. Descriptive statistics were used to describe the characteristics of the curricula.

\section{Results}

The search yielded 32,822 papers. An additional 13 papers were identified by hand searching relevant oncological organizations. This yielded a total of 32,835 papers. 9,952 duplicates were identified and removed. The remaining 22,883 papers were then reviewed against the inclusion and exclusion criteria. 22,554 papers were excluded following this review. Full abstracts for the remaining 329 papers were obtained and reviewed. 281 papers were excluded leaving 48 papers that underwent full text review. Ultimately 17 of these papers met the inclusion criteria and formed the bases for analysis for this study. See Figure 1.

\section{What global curricula exist for oncology}

Seventeen curricula were identified: 5 (29\%) were from medical oncology, 5 (29\%) were from radiation oncology, 5 (29\%) were from surgical oncology, 1 (6\%) from thoracic oncology and 1 (6\%) from clinical oncology. Most of the curricula were published after 2000. 11 (65\%) were published from 2010 to 2018. Table 2 summarizes the curricular details.

\section{Who developed these curricula}

The majority of these curricula, $13(68 \%)$, originated from Europe. The 17 curricula had a total of 300 (mean 19; range 4-98) authors. The majority of the authors were male $(n=207$, $69 \%)$. Most authors were from either Europe $(n=190 ; 64 \%)$ or North America $(n=73 ; 24 \%)$. Table 3 summarizes the author characteristics.

When the curricula were analyzed by the year of publication we identified a trend of increasing proportion of female authors from a mean of $17 \%$ in the oldest publications to a mean of $37 \%$ female authors in the more recently published curricula. In addition, the geographic distribution of authors represented in the publications increased from 1 country in the oldest publication to a mean of 9 countries represented in the most recent curricula. See Table 4. 

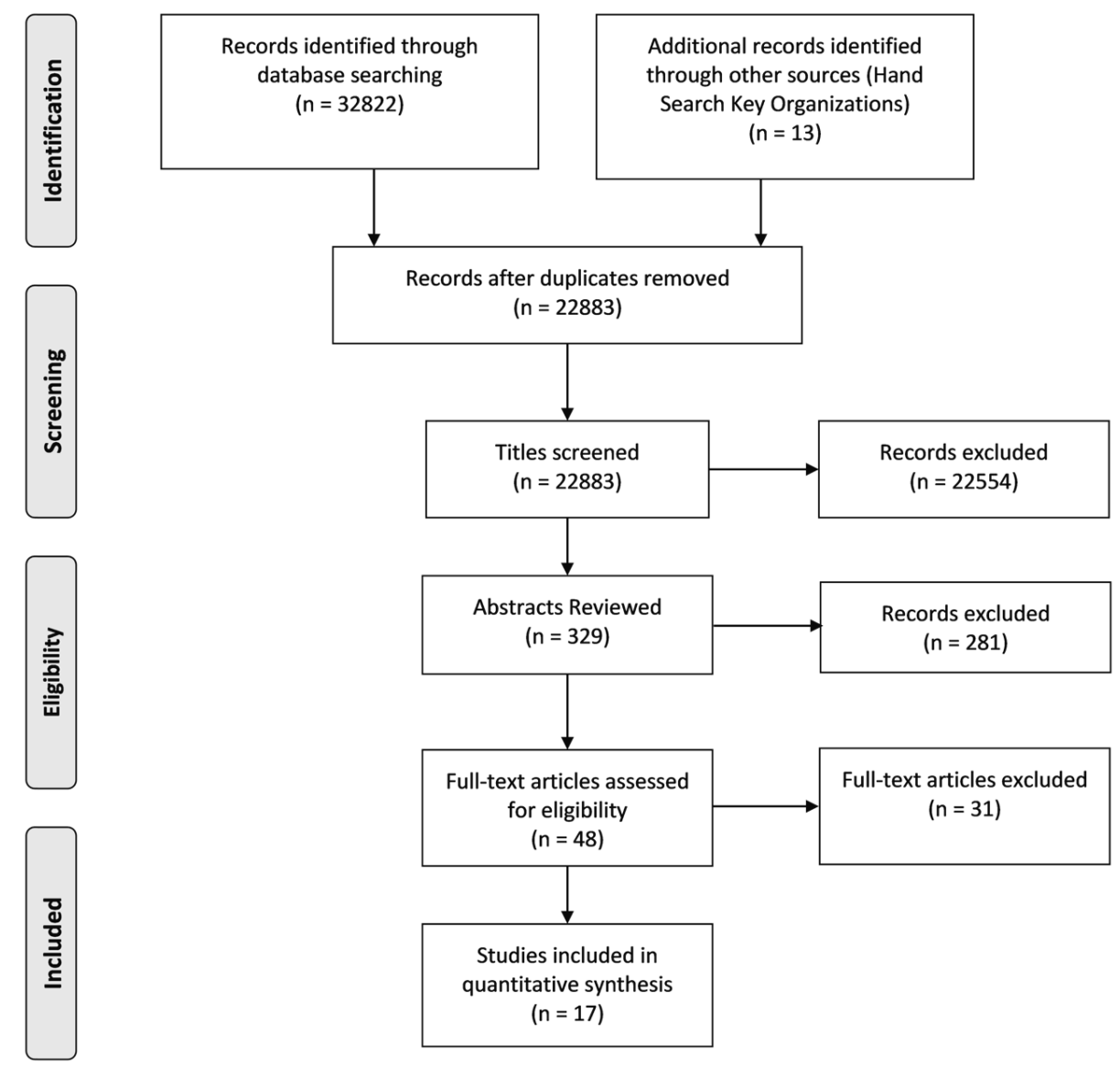

Figure 1: PRISMA 2009 Flow Diagram

Table 2: Curricular Characteristics

\begin{tabular}{ll}
\hline Medical Specialty & $1(6 \%)$ \\
\hline Clinical oncology & $5(29 \%)$ \\
\hline Medical oncology & $5(29 \%)$ \\
\hline Radiation oncology & $5(29 \%)$ \\
\hline Surgical oncology & $1(6 \%)$ \\
\hline Thoracic oncology & \\
\hline Publication Year & $1(6 \%)$ \\
\hline $1980-1989$ & $0(0 \%)$ \\
\hline $1990-1999$ & $5(29 \%)$ \\
\hline $2000-2009$ & $11(65 \%)$ \\
\hline $2010-2018$ & \\
\hline Region of Publication & $0(0 \%)$ \\
\hline Africa & $0(0 \%)$ \\
\hline Asia & $2(11 \%)$ \\
\hline Oceania & $13(68 \%)$ \\
\hline Europe & $0(0 \%)$ \\
\hline Latin Americas & $4(21 \%)$ \\
\hline North America &
\end{tabular}

* 2 curricula were attributed to two regions equally (19 regions for 17 publications) 
Table 3: Author Characteristics

\begin{tabular}{ll}
\hline Oncology Sub-Specialty & \\
\hline Clinical oncology & $11(4 \%)$ \\
\hline Hematology oncology & $26(9 \%)$ \\
\hline Medical oncology & $56(19 \%)$ \\
\hline Oncology (other) & $21(7 \%)$ \\
\hline Radiation oncology & $50(17 \%)$ \\
\hline Radiation physics & $2(1 \%)$ \\
\hline Radiation therapy & $15(5 \%)$ \\
\hline Surgical oncology & $40(14 \%)$ \\
\hline Thoracic oncology & $9(3 \%)$ \\
\hline Other & $57(20 \%)$ \\
\hline Unknown & $3(1 \%)$ \\
\hline Gender & \\
\hline Female & $92(31 \%)$ \\
\hline Male & $207(69 \%)$ \\
\hline Data not available & 1 \\
\hline Region & \\
\hline Africa & $0(0 \%)$ \\
\hline Asia & $9(3 \%)$ \\
\hline Oceania & $19(6 \%)$ \\
\hline Europe & $190(64 \%)$ \\
\hline North America & $73(1 \%)$ \\
\hline South America & \\
\hline Unknown & \\
\hline & \\
\hline
\end{tabular}

Table 4: Curricular Trends over Time

\begin{tabular}{llllll}
\hline $\begin{array}{l}\text { Publication } \\
\text { Year }\end{array}$ & $\begin{array}{l}\text { Number of } \\
\text { publications }\end{array}$ & $\begin{array}{l}\text { Number of } \\
\text { Authors } \\
\text { Mean (Range) }\end{array}$ & $\begin{array}{l}\text { Male Authors } \\
\text { Mean (Range) }\end{array}$ & $\begin{array}{l}\text { Female Authors } \\
\text { Mean (Range) }\end{array}$ & $\begin{array}{l}\text { Countries Represented } \\
\text { in Authorship Mean } \\
\text { Range) }\end{array}$ \\
\hline $1980-1989$ & 1 & 6 & $83 \%$ & $17 \%$ & 1 \\
\hline $1990-1999$ & 0 & N/A & N/A & N/A & N/A \\
\hline $2000-2009$ & 5 & $13(6-26)$ & $79 \%(61-86 \%)$ & $21 \%(14-39 \%)$ & $6(1-15)$ \\
\hline $2010-2018$ & 11 & $23(4-98)$ & $63 \%(42-88 \%)$ & $37 \%(12-58 \%)$ & $9(1-23)$ \\
\hline
\end{tabular}

\section{What was the purpose for these curricula}

The most common purpose for these curricula were promoting or improving the quality of patient care $(n=11)$, the harmonization of training standards $(n=10)$, and facilitating physician mobility across countries $(n=3)$.

\section{What methods were used to develop the curricula}

Eleven of the seventeen curricula describe the process used in their development of the curricula. The methods for creation of these curricula were most commonly a committee or task force $(n=10)$ and one was created using a modified Delphi process. All curricula 


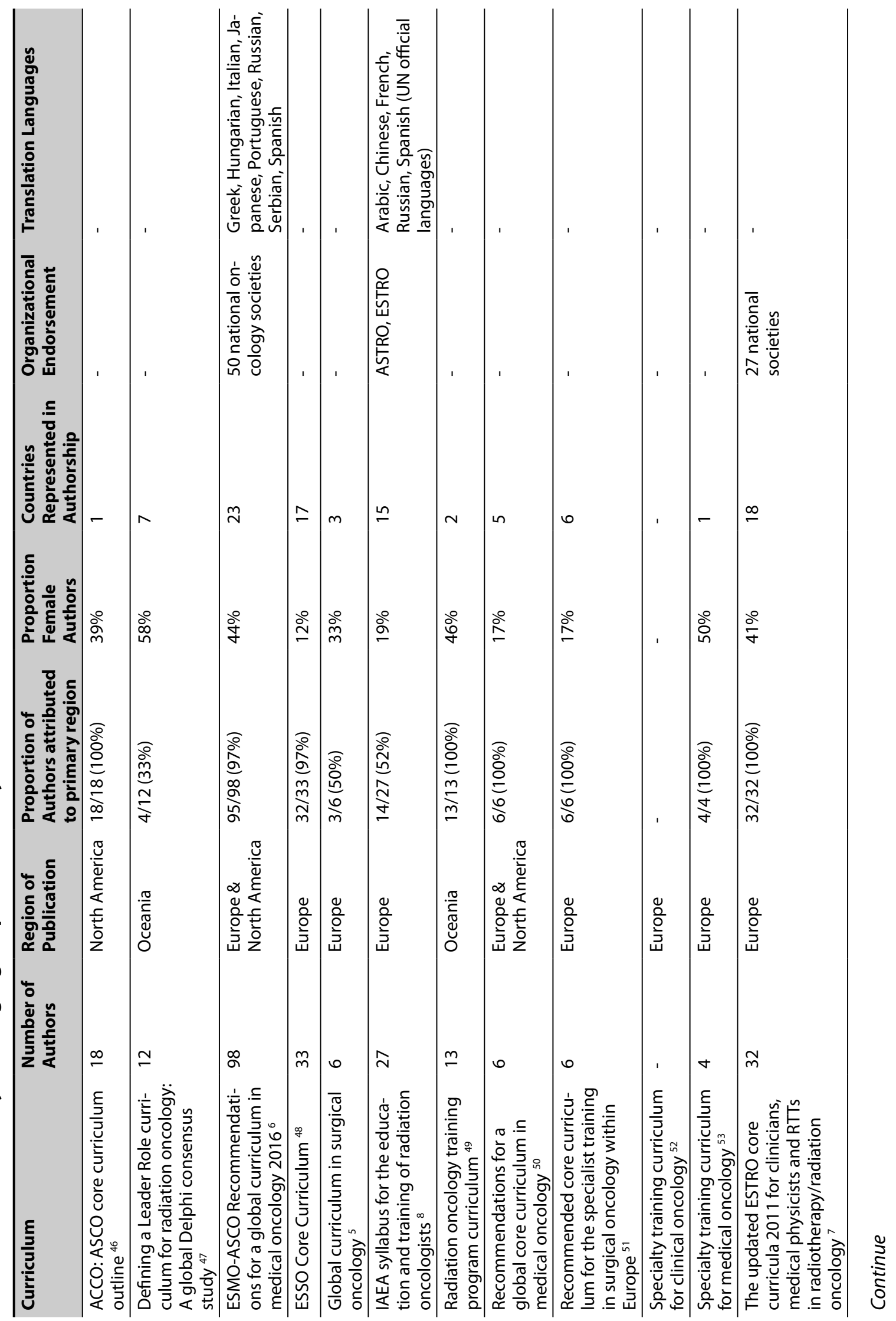




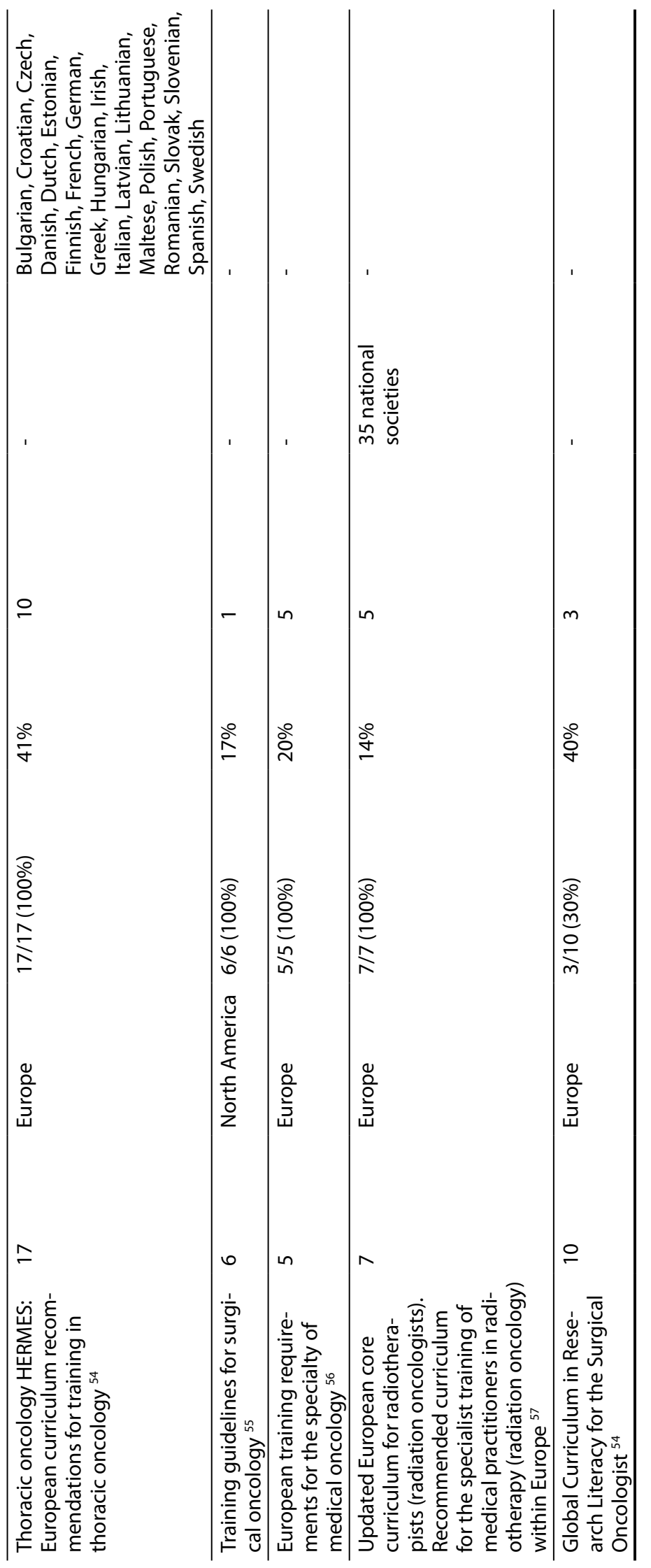


were published in English. However, three provided official translation into 23, 8 and 5 languages respectively. Four curricula describe to what extent they have been externally endorsed. These data are summarized in Table 5.

\section{Discussion}

This theoretically framed systematic review of global oncology curricula has identified that the majority of these curricula originated from Western regions, were published in English for the primary document and are dominated by male authorship. We have demonstrated that there is an effort to include authors from varied global regions and women in the development of these global curricula in oncology as well as efforts to overcome the limitations of English publication through official translated documents. However, there remains a disproportional representation of Western authors participating in these consensus processes. This work sought to report, through an anti-colonial lens, a curricular review concept proposed by Bleakley et al by reflecting on "what we are about when we design a programme of education" ${ }^{\prime 30}$. As stated by Bleakley et al curricula, created through consensus agreement, are a human creation and therefore are influenced by those individuals' interests and ideologies ${ }^{30}$. Using a European curricula as an example, a predominantly European representation on regional curricula for Europe is expected, it still remains important to explore the issue of regional representation given the increasing numbers of migrants and refugees around the world. Using the ESTRO Core curriculum as an example there was an increase in representation from 7 countries in 2004 to 32 countries in the 2011 version?. However, from our data we were not able to address the degree to which there was equity of contribution to the final curricular product by the various countries represented in the authorship, or what if any differences in opinion may have occurred during the deliberations. Addressing these details would be an important focus of future research best served by a qualitative approach.

The representation of female authors in the development of these curricula has increased over time. The proportion of women, while still under-represented compared to male authors, rose from $17 \%$ in the oldest curricula to a mean of $37 \%$ in the most recently published curricula. Women represent the majority of the global healthcare workforce, in Western and non-Western settings ${ }^{33}$, as well as a growing proportion of the oncology workforce ${ }^{34}$ and their underrepresentation in the development of global curricula is concerning. Ensuring representation of women, as they are delivering the majority of healthcare globally as well as representing a growing proportion of the oncology workforce, may be a factor in mitigating the perceived mismatch between curricula and desired competencies for clinical practice.

Although the training and credentialing of physicians remains largely a nation-bound activity currently, higher-education environments are simultaneously global, national 
and local ${ }^{35}$. Promoting physician mobility was one of the main purposes of these curricula identified in our study. Identifying this as a priority may reflect the decline of the nationstate in the face of a globalizing workforce ${ }^{36}$. The ability to move between nations promotes brain circulation and may reduce brain waste ${ }^{9,37}$. This phenomenon is well recognized in the Canadian context where immigrant physicians were the least likely to be employed ${ }^{37}$. In oncology, where the profound lack of qualified oncologists in the face of a rapidly growing cancer population is resulting in serious gaps in cancer care, efforts to ensure all qualified physicians are seeing patients is laudable. However, caution is needed as there are many examples where mobility of credentials results in brain drain from low and middle income countries and may widen the gaps in access to care in areas that need it the most ${ }^{9}$. Models of training that address local needs, provide high standards of care and promote local and/or regional retention are desirable and have been achieved in areas such as psychiatry ${ }^{38}$.

Much global health work is premised on study, research and practices that aim to improve health for all people ${ }^{39}$ with an implicit assumption that care needs of cancer patients will be the same anywhere in the world. Eleven of the curricula in our analysis identified improving the quality of patient care as their purpose. Given the composition of the curricular committees that generate these materials, the effort may be perpetuating dominant Western discourses in medical care and training. A dominant discourse is "a particular language and a distinctive worldview in which some things are regarded as inherently more important or true than others" ${ }^{40}$. If we consider that the curricula identified in our work are mostly created by Western organizations, or certain dominant countries within a specific region, and engage to varying degrees' authors, contributors and endorsements from other regions one questions to what degree dominant discourses of Western medical and educational priorities are imposed in regions across the globe. We must question how differing global interests and priorities are represented in these working groups; previous colonial structures can be reproduced in modern encounters ${ }^{41}$. We must continue to question how current curricula development practices, which largely rely on consensus work through committees may silence or otherwise poorly represent alternative or minority perspectives ${ }^{42}$. The discourse of the West improving patient care in the Global South is dominant in the literature. The non-Western perspectives are considered less knowledgeable about medical education and this may contribute to the disproportional representation of Western authors in global curricular efforts ${ }^{41}$.

Our study has several limitations. One limitation of this work is the phenomenon of the marginalization of non-English language scholarship ${ }^{35}$. All of the curricula identified in this work were developed and initially published in English. Subsequently two were officially translated into five and eight languages respectively. The impact of the dominance of English-language in the global space of curriculum development requires further study as 
it is possible that the predominance of English language marginalizes other perspectives and does not incorporate regional or local practices ${ }^{35}$. This use of English to develop and disseminate the curricula for 'quality' training may also promote neocolonialism. Another limitation of our work, which utilized a systematic review strategy, is that of publication bias. The dominance of Western perspectives in the published literature is well known including in the field of global oncology ${ }^{3}$. The authors were conscious of the limitations and utilized hand searches of international groups in oncology as well as contacting members of these organizations including those that are not English speaking as their primary language. However, using the published literature as a focus for our work has allowed us to make explicit imbalances in representation that compromise sated efforts to produce curricula that are globally applicable. The methodology of a systematic review does not allow us to capture complex socio-political relationships at play in the development of global curricula. As the field struggles with questions of building capacity in oncology treatment around the world, future research should consider studying process and implementation issues using methods that are specifically designed to capture power issues. Another limitation is our categorization of different regions of the world as Western and nonWestern. We acknowledge, while this facilitates the analysis, it is an oversimplification of the diversity of many countries which constitute these regions. Nevertheless, the sociopolitical history of medical fields has largely favoured Western high resource regions of the world and as our study shows, representing other perspectives and experiences required deliberate effort. In addition, we were not able to perform an intersectionality analysis to explore if the increase in female representation is dominated by a rise only in Western female participation ${ }^{43}$. This would be an important area for future work. Finally, we are not able to draw conclusions about the degree to which the representation bias is reflected in the content of the existing curricula, and hence the degree to which this content reflects the healthcare and health-system priorities in diverse geographic settings. We also were unable to report on the nature in which these curricula are actually implemented in local contexts and the degree of local customizations that occurs, including the incorporation of alternative, indigenous health approaches, to better address local health care needs is also not known. Further studies that employ a qualitative methodology would be better suited to addressing these critical considerations.

\section{Conclusions}

Using a critical, anticolonial lens we have reported the Western, male influence in the creation of global oncology curricula. We suggest, that as a result, these curricula may not incorporate relevant socio-cultural perspectives impacting care in diverse geographic settings. 


\section{References}

1. Frenk J, Chen L, Bhutta ZA, Cohen J, Crisp N, Evans T, et al. Health professionals for a new century: transforming education to strengthen health systems in an interdependent world. The Lancet. 2010;376(9756):1923-58.

2. Hollenberg D, Muzzin L. Epistemological Challenges to Integrative Medicine: An anti-colonial perspective on the combination of complementary/alternative medicine with biomedicine. Health Sociology Review. 2010;1(19).

3. Fouad T. Academic Dependency: A postcolonial critique of global health collaborations in oncology. Medicine Anthropology Theory. 2018;5(2):127-41.

4. Burmeister J, Chen Z, Chetty I, Dieterich S, Doemer A, Dominello M, et al. The American Society for Radiation Oncology's 2015 Core Physics Curriculum for Radiation Oncology Residents. 2016;95(4):1298-303.

5. Are C, Berman R, Wyld L, Cummings C, Lecoq C, Audisio RA. Global curriculum in surgical oncology. European journal of surgical oncology : the journal of the European Society of Surgical Oncology and the British Association of Surgical Oncology. 2016;42(6):754-66.

6. Dittrich C, Kosty M, Jezdic S, Pyle D, Berardi R, Bergh J, et al. ESMO / ASCO Recommendations for a Global Curriculum in Medical Oncology Edition 2016. 2016.

7. Eriksen JG, Beavis AW, Coffey MA, Leer JWH, Magrini SM, Benstead K, et al. The updated ESTRO core curricula 2011 for clinicians, medical physicists and RTTs in radiotherapy/radiation oncology. Radiotherapy and oncology : journal of the European Society for Therapeutic Radiology and Oncology. 2012;103(1):103-8.

8. International Atomic Energy Agency (IAEA). IAEA Syllabus for the Education and Training of Radiation Oncologists (2009); (Training Course Series No. 36). http://www-pub.iaea.org/MTCD/ Publications/PDF/TCS-36_web.pdf. Accessed April 10, 2019

9. Spring J. Globalization of Education: An Introduction. 2nd ed. New York: Routledge; 2014.

10. Vinokur A. Brain Migration Revisited. Globalisation, Societies and Education. 2006;4(1):7-24.

11. Lofters A, Slater M, Fumakia N, Thulien N. "Brain drain" and "brain waste": experiences of international medical graduates in Ontario. Risk Manag Healthc Policy. 2014;7:81-9.

12. Atun R, Jaffray DA, Barton MB, Bray F, Baumann M, Vikram B, et al. Expanding global access to radiotherapy. The Lancet Oncology. 2015;16(10):1153-86.

13. Martimianakis MA, Hafferty FW. The world as the new local clinic: a critical analysis of three discourses of global medical competency. Social science \& medicine. 2013;87:31-8.

14. Royal College of Physicians and Surgeons of Canada. Royal College International (2020). http://www.royalcollege.ca/rcsite/international-e. Accessed January 22, 2020

15. Best Evidence in Medical and Health Professional Education. The BEME Collaboration (2020) https://www.bemecollaboration.org/. Accessed January 22, 2020

16. Sefton AJ. New approaches to medical education: an international perspective. Medical principles and practice : international journal of the Kuwait University, Health Science Centre. 2004;13(5):239-48. 
17. Jha V, McLean M, Gibbs TJ, Sandars J. Medical professionalism across cultures: a challenge for medicine and medical education. Medical teacher. 2015;37(1):74-80.

18. Sallie A Marston KW, John Paul Jones. Flattening Ontologies of Globalization: THe Nollywood Case. Globalizations. 2007;4(1):45-63.

19. American Society of Clinical Oncology (ASCO). ASCO Annual Meeting (2020). https://www. asco.org/. Accessed June 252017

20. American Society for Radiation Oncology (ASTRO). ASTRO Targeting Cancer Care (2020). https://www.astro.org/home/. Accessed June 252017

21. European Society for Radiotherapy \& Oncology (ESTRO). ESTRO (2020). http://estro.org/. Accessed June 242017

22. African Organization for Research \& Training in Cancer (AORTIC). AORTIC (2001). http://www. aortic-africa.org. Accessed July 1, 2018

23. The Royal Australian and New Zealand College of Radiologists (RANZCR). (2020). http://www. ranzcr.com. Accessed June 24, 2017

24. Federation of Asian Organizations for Radiation Oncology (FARO). What's new: FARO Meeting 2019 (2019). http://www.faroac.org/ Accessed Jan 7, 2020

25. Asociación Ibero Latinoamericana de Terapia Radiante Oncológica (ALATRO). ALATRO (2019). http://www.alatro.org/. Accessed Jan 7, 2020

26. Canadian Association of Radiation Oncology (CARO). (2016). http://www.caro-acro.ca/. Accessed June 252017

27. European Society for Medical Oncology (ESMO). European Society for Medical Oncology (ESMO) (2020). http://www.esmo.org/. Accessed June 242017

28. International Atomic Energy Agency (IAEA). (2019). https://www.iaea.org/. Accessed June 24 2017

29. Keeling A. A New Gender Majority in the WHO Leadership Team Announced or Man Bites Dog in WHO. (2017). https://www.womeningh.org/single-post/2017/11/08/A-New-Gender-Majority-in-the-WHO-Leadership-Team-Announced-or-Man-Bites-Dog-in-WHO. Accessed June 24, 2017

30. Bleakley A, Brice J, Bligh J. Thinking the post-colonial in medical education. Medical education. 2008;42(3):266-70.

31. Whitehead CR. On gunboats and grand pianos: medical education exports and the long shadow of colonialism. Advances in health sciences education : theory and practice. 2016;21(1):14.

32. NVivo Qualitative Data Analysis Software. QSR International Pty Ltd. Version 11, 2015. Available from: http://www.qrsinternational.com/nvivo.

33. Langer A, Meleis A, Knaul FM, Atun R, Aran M, Arreola-Ornelas $\mathrm{H}$, et al. Women and health: the key for sustainable development. The Lancet. 2015;386(9999):1165-210.

34. American Society of Clinical Oncology. The state of cancer care in America, 2017: A report by the American Society of Clinical Oncology. Journal of oncology practice. 2017;13(4):e353-e94. 
35. Marginson S. Global field and global imagining: Bourdieu and worldwide higher education. British Journal of Sociology of Education. 2008;29(3):303-15.

36. Scholte JA. Globalization: A critical introduction. 2nd ed: Macmillan International Higher Education; 2005.

37. Goldberg MP. Discursive policy webs in a globalisation era: a discussion of access to professions and trades for immigrant professionals in Ontario, Canada. Globalisation, Societies and Education. 2006;4(1):77-102.

38. Whitehead C, Wondimagegn D, Baheretibeb Y, Hodges B. The International Partner as Invited Guest: Beyond Colonial and Import-Export Models of Medical Education. Academic Medicine. 2018;93(12):1760-3.

39. Koplan JP, Bond TC, Merson MH, Reddy KS, Rodriguez MH, Sewankambo NK, et al. Towards a common definition of global health. The Lancet. 2009;373(9679):1993-5.

40. Brookfield $\mathrm{S}$. The power of critical theory for adult learning and teaching. 1st ed. Columbus, $\mathrm{OH}$ : Open University Press; 2005.

41. Hanson L, Cheng J. Production of the Global Health Doctor: Discourses on International Medical Electives. Engaged Scholar Journal: Community-Engaged Research, Teaching, and Learning. 2018;4(1):161-80.

42. Spivak GC. In other worlds: Essays in cultural politics: Routledge; 2012.

43. Tsouroufli M, Rees CE, Monrouxe LV, Sundaram V. Gender, identities and intersectionality in medical education research. Medical education. 2011;45(3):213-6.

44. PG A. Education and neocolonialism. In: Ashcroft B GG, Tiffin H, editor. The post-colonial Studies Reader. London: Routledge; 2004. p. 452-6.

45. Sharma M. Çan the Patient Speak?': postcolonialism and patient involvement in undergrduate and postgraduate medical education. Medical education. 2018;52:471-9.

46. Muss HB, Von Roenn J, Damon LE, Deangelis LM, Flaherty LE, Harari PM, et al. ACCO: ASCO core curriculum outline. Journal of clinical oncology. 2005;23(9):2049-77.

47. Turner S, Seel M, Trotter T, Giuliani M, Benstead K, Eriksen JG, et al. Defining a Leader Role curriculum for radiation oncology: A global Delphi consensus study. Radiotherapy and Oncology. 2017;123(2):331-6.

48. Audisio R NP, Poston G, Wyld L. ESSO Core Curriculum 2013. European journal of surgical oncology : the journal of the European Society of Surgical Oncology and the British Association of Surgical Oncology. 2013;39(S1):S1-S32.

49. Turner $S M C$, Longergan $D$, Holt $T$, Lehman $M$, James $M$, Lee $M$, Penniment $M$, Yuile $P$, Kok $D$, Lee A, Ng E, Allen A. Radiation Oncology Training Program Curriculum (2012). https://www.ranzcr. com/college/document-library/radiation-oncology-training-program-curriculum. Accessed June 25, 2017

50. ESMO/ASCO Task Force on Global Curriculum in Medical Oncology, Hansen HH, Bajorin DF, Muss HB, Purkalne G, Schrijvers D, et al. Recommendations for a global core curriculum in medical oncology. Annals of Oncology. 2004;15(11):1603-12. 
51. Naredi P, Leidenius M, Hocevar M, Roelofesen F, van de Velde C, Audisio RA. Recommended core curriculum for the specialist training in surgical oncology within Europe. Surgical oncology. 2008;17(4):271-5.

52. The Royal College of Radiologists. Specialty Training Curriculum for Clinical Oncology. London, England, The Faculty of Clinical Oncology; 2016.

53. Joint Royal College of Physicians Training Board. Specialty Training Curriculum for Medical Oncology. London, England; 2017.

54. Are C, Yanala U, Malhotra G, Hall B, Smith L, Wyld L, Cummings C, Lecoq C, Audisio RA, Berman RS. Global Curriculum in Research Literacy for the Surgical Oncologist. Ann Surg Oncol. 2018 Mar; 25(3):604-616.

55. Schweitzer RJ, Edwards MH, Lawrence Jr W, Mozden PJ, Scanlon EF, Leffall Jr LD. Training guidelines for surgical oncology. Cancer. 1981;48(10):2336-40.

56. Rauh S NO, Brandao M, Colomer R, Turhal S. European training requirements for the specialty of medical oncology. Brussels, Belgium; 2017.

57. Baumann M, Leer J, Dahl O, De Neve W, Hunter R, Rampling R, et al. Updated European core curriculum for radiotherapists (radiation oncologists). Recommended curriculum for the specialist training of medical practitioners in radiotherapy (radiation oncology) within Europe. Radiotherapy and oncology. 2004;70(2):107-13. 


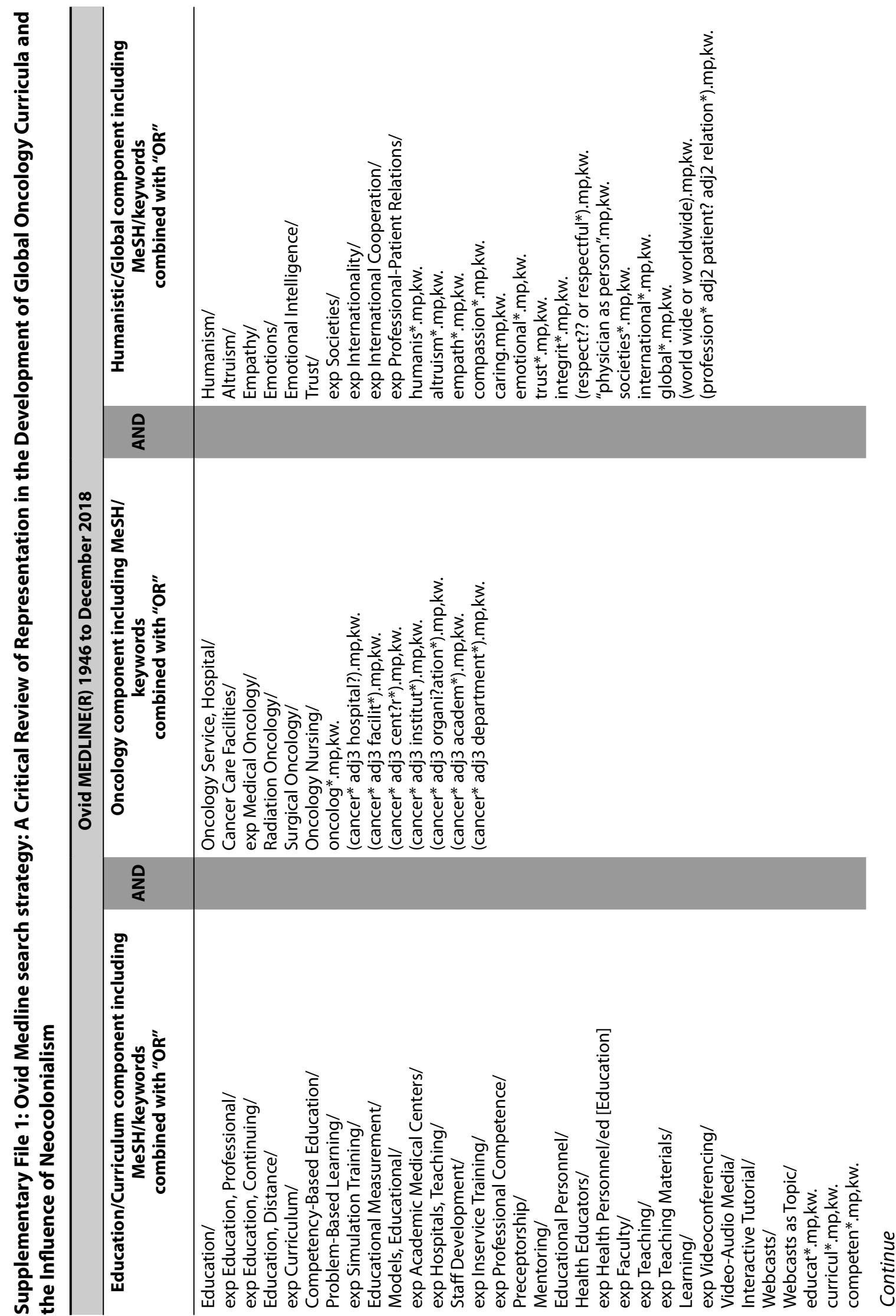




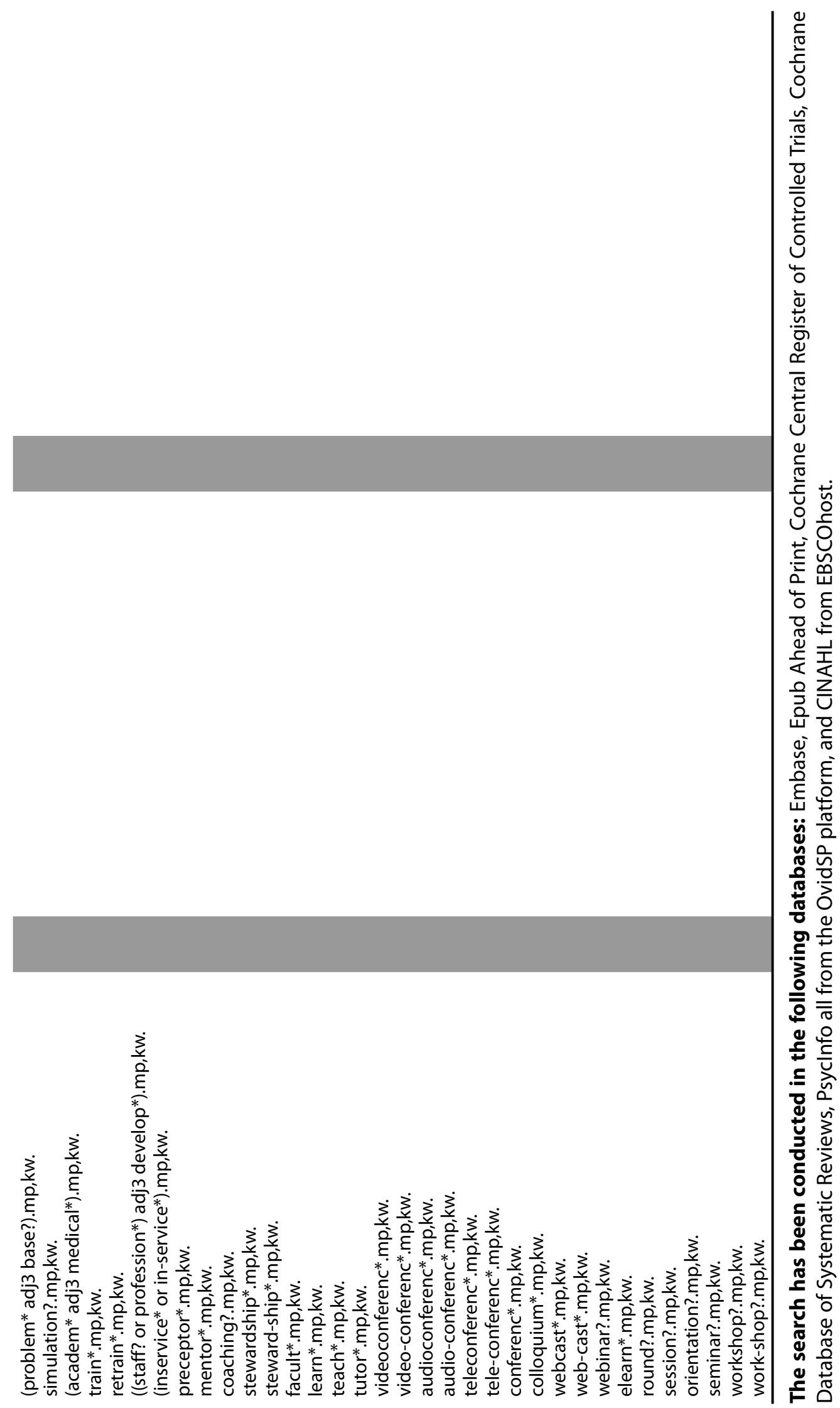


CHAPTER 4

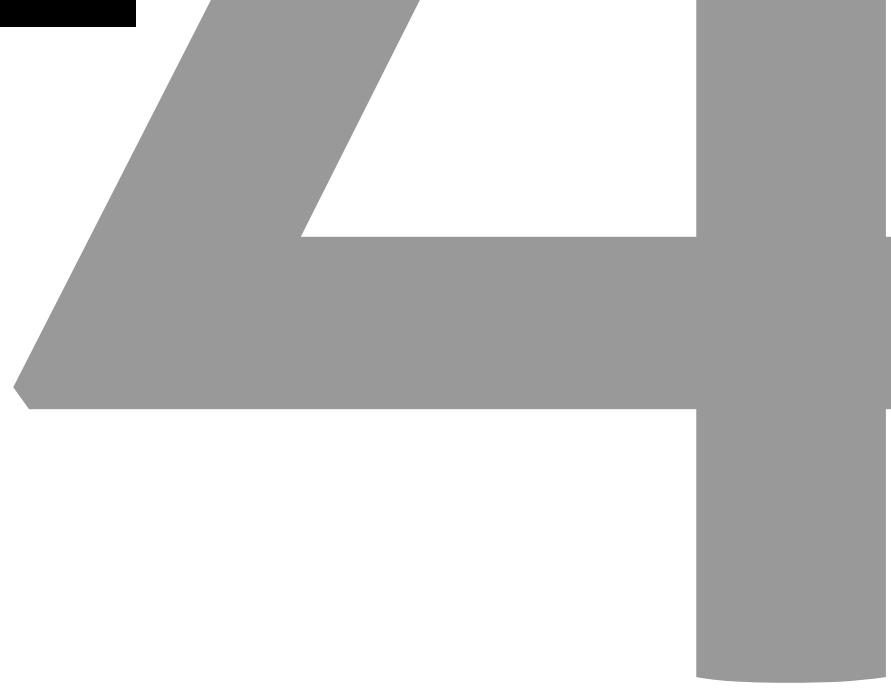




\section{Humanism in Global Oncology Curricula: An Emerging Priority}

Published as: Giuliani M, Martimianakis MA, Broadhurst M, Papadakos J, Fazelad R, Driessen E, Frambach J. Humanism in global oncology curricula: an emerging priority. Curr Oncol. 2020 


\section{Introduction}

Training in humanism provides the skills important for improving the quality of care received by patients, achieving shared decision making with patients and navigating systems level challenges. However, there is potentially a lack of attention to humanistic competencies in global oncology curricula due to the dominance of the biomedical model. This study aimed to explore the incorporation of humanistic competencies in global oncology curricula.

\section{Materials and methods}

Seventeen global oncology curricula formed the basis of this analysis. A curricular item was coded as either humanistic (as defined by the I.E.C.A.R.E.S. framework) or non-humanistic. If identified as humanistic the aspect of humanism from the I.E.C.A.R.E.S. framework, such as Altruism, was coded. All items, humanistic and not, were coded by the CanMEDS framework as one of the seven CanMEDS competency domains of Medical Expert, Communicator, Collaborator, Leader, Scholar, Professional or Health Advocate.

\section{Results}

7792 curricular items, in 17 curricula, were identified and 780 (10\%) aligned with the I.E.C.A.R.E.S humanism framework. The proportion of humanistic items in individual curricula ranged from $2 \%$ to $26 \%$ and the proportion increased from $3 \%$ in the oldest to $11 \%$ in the most recent curricula. Of the humanistic items $35 \%$ were coded under Respect, 31\% under Compassion, 24\% under Empathy, 5\% were under Integrity, 2\% under Excellence, 1\% under Altruism, and 1\% under Service. Within the CanMEDS domains, the majority of humanistic items aligned with Professional (35\%), Medical Expert (31\%) or Communicator (25\%).

\section{Conclusion}

The proportion of humanistic competencies has been increasing in global oncology curricula over time, however the overall proportion remains low and represents a largely Western perspective on what constitutes humanism in healthcare. The representation of humanism primarily focuses on the I.E.C.A.R.E.S attributes of Respect, Compassion and Empathy. 


\section{Introduction}

Calls for curricular reform to focus on person-centered care are growing and the concept of humanism is at the center of these discussions. ${ }^{1,2}$ The concept of humanism is evolving in medical education literature but has been a core element of the medical profession since its inception. ' A broad conceptualization of humanism in medicine is "any system or mode of thought or action in which human interests, values, and dignity predominate."1 A more specific definition describes humanism in healthcare as "a respectful and compassionate relationship between physicians, as well as all other members of the healthcare team, and their patients. It reflects attitudes and behaviours that are sensitive to the values and the cultural and ethnic backgrounds of others." ${ }^{\prime 3}$ This definition is the basis of the Gold Foundation framework of humanism, which describes seven attributes of the humanistic healthcare professional: Integrity, Excellence, Collaboration \& Compassion, Altruism, Respect \& Resilience, Empathy and Service (I.E.C.A.R.E.S.). ${ }^{3}$ Table I lists definitions of these attributes. In line with these attributes, a humanistic physician has been described as one who considers the influence of social, cultural and spiritual experiences in patient care. ${ }^{4}$

Table I: I.E.C.A.R.E.S Framework from the Gold Foundation (Arnold P. Gold Foundation 2018) ${ }^{3}$

\begin{tabular}{ll}
\hline Integrity & The congruence between expressed values and behavior \\
\hline Excellence & clinical expertise \\
\hline Collaboration \& Compassion & $\begin{array}{l}\text { The awareness and acknowledgement of the suffering of another and the } \\
\text { desire to relieve it }\end{array}$ \\
\hline Altruism & The capacity to put the needs and interests of another before your own \\
\hline Respect \& Resilience & The regard for the autonomy and values of another person \\
\hline Empathy & The ability to put oneself in another's situation, e.g., physician as patient \\
\hline Service & $\begin{array}{l}\text { The sharing of one's talent, time and resources with those in need; giving } \\
\text { beyond what is required }\end{array}$ \\
\hline
\end{tabular}

Training physicians as scientists is central to the biomedical model of medical education ${ }^{5}$ including oncology education and training. However, centering the role of science in medical training may also inadvertently undermine the role of humanism in Western medical practice. ${ }^{6}$ In cancer care, where communication skills and team-based care are essential skills, training in humanism may have particular relevance. In addition, ongoing and forthcoming changes to professional practice, such as artificial intelligence, and the implementation of curricula in global contexts may also challenge the capacity of providers to practice humanistic care. For example, artificial intelligence has the potential to radically change the nature of medical practice by replacing large portions of the diagnostic work currently done by physicians. ${ }^{7}$ Such changes in the delivery of care hold implications for patient interactions with health care providers how they experience their overall care. Furthermore, it has been argued that, similar to other healthcare professionals, oncologists are not receiving the training they need to meet the needs of all patients, families and the healthcare system. ${ }^{8}$ This mismatch between training and clinical 
practice, including gaps in team-based competencies and communication skills has been attributed to outdated curricula, ${ }^{8}$ and potentially a lack of attention to humanistic competencies, such as an awareness of how context and culture impacts health care behaviours, experiences and outcomes.

The high rates of burn-out amongst oncologists call for a different approach to education. ${ }^{9}$ Training in humanism has been argued to increase physician job satisfaction ${ }^{10}$ and improve both patient clinical outcomes and satisfaction with their care ${ }^{7}$ as well as to provide health professionals with the skills to achieve shared decision making with patients and their families, to navigate systems level challenges, and to function positively within the healthcare team. ${ }^{6}$ The skills afforded by humanistic education including engaging with complexity and ambiguity, mitigating physician burn-out and navigating power relationships may be critical in closing the current training to practice gap that has been identified for health care curricula. ${ }^{11}$

Despite growing recognition of the potential of humanism for medical education, current understanding of the integration of humanism into curricula is limited, specifically in light of increasing efforts to establish and implement global curricula. Global oncology curricula have been identified for oncology specialities including radiation, medical and surgical oncology. The purpose of these curricula is to improve the quality of patient care; harmonize training standards across jurisdictions; and ultimately to facilitate physician mobility (Giuliani M, Frambach J, Broadhurst M, Papadakos J, Driessen E, Martimianakis T. A Critical Review of Representation in Global Oncology Curricula Development and the Influence of Neocolonialism. In preparation). This may be problematic considering that understanding of humanism and its integration in curricula is culturally specific., 2, Humanistic competencies, such as ethics and altruism, are socially constructed ideas and practices and therefore the way in which they are conceptualized, performed and received can vary across different regions. ${ }^{13}$ As a result, adopting humanistic competencies from Western to non-Western contexts ${ }^{14}$ is a difficult educational process. A known challenge in establishing global curricula is the tension between meeting local needs and achieving international standards. ${ }^{15,16}$ This challenge of balancing the local and the global is particularly effortful for humanistic competencies. ${ }^{17}$

Understanding the current state of the integration of humanism in global oncology curricula may yield insight into a possible source of the mismatch between curricula and the competencies needed for practice. There are growing efforts to internationalize curricula and there is a potential for an overdominance of Western orientation at the expense of other perspectives in these efforts. While there is no clear consensus on how to prioritize curricular content, having a greater understanding of the content of existing curricula can assist in informing future work in global jurisdictions to prepare healthcare 
professionals for practice. The aims of this study were to explore to what extent humanistic competencies are included in global oncology curricula, and to identify the nature of the included humanistic competencies.

\section{Methods}

\section{Sampling}

This study involved an analysis of the content of published global oncology curricula. Seventeen global oncology curricula identified in a prior manuscript formed the basis for this analysis (Giuliani M, Frambach J, Broadhurst M, Papadakos J, Driessen E, Martimianakis T. A Critical Review of Representation in Global Oncology Curricula Development and the Influence of Neocolonialism. In preparation). The publication by Giuliani et al identified seventeen global oncology curricula through a systematic review. Of 17 curricula 5 were from medical oncology, 5 were from radiation oncology, and 4 were from surgical oncology. To analyze these curricula, we utilized two well-known and internationally recognized medical competency frameworks as part of this analysis; the Gold Foundations' I.E.C.A.R.E.S framework (Table I) and the CanMEDS framework. Key word codes were derived from the components of the I.E.C.A.R.E.S framework and were assigned to each curricular item. As such, the keyword codes consisted of the Integrity, Excellence, Collaboration \& Compassion, Altruism, Respect \& Resilience, Empathy and Services.

We also coded the curricula according to the CanMEDS competency framework. ${ }^{18}$ CanMEDS was selected as it has been implemented or adopted in multiple jurisdictions around the world and it aligns well with other competency frameworks like the Accreditation Council for Graduate Medical Education framework. In addition, CanMEDS has detailed elaboration of the components and attributes attributed to each of the seven competency domains that allowed for appropriate application of the framework to the curricular documents. As a Western framework with broad uptake around the world, its application allowed us to understand one system through which medical education is currently operationalized in the global context. By coding according to CanMEDS we were able to assess what areas may be currently overregulated or emphasized compared to other areas in regions of the world adopting CanMEDS. These data could provide insight into how oncology education has organized and prioritized curricular content.

We hypothesized that there would be a relative lack of humanism in global curricula and that this may signal an under-emphasis of humanistic issues in curriculum forums where Western voices dominate or a limited ability to attend to the complexity of including such competencies in global curricula. 


\section{Curricular Content Analysis}

A priori, a coding structure was determined. Two reviewers (MG and MB) discussed over several meetings the application of the I.E.C.A.R.E.S. and CanMEDS frameworks to the curricula. The analysis as it progressed was discussed with other authors at regular meetings. The two investigators then independently reviewed each curricular document. Consensus was reached on the nature of each competency item with any disagreements resolved by adjudication by the whole research team as necessary. The analysis was performed using NVivo version $11 .{ }^{19}$

Each curricular item was coded as either humanistic (as defined by the I.E.C.A.R.E.S. framework) or non-humanistic. If identified as humanistic the aspect of humanism from the I.E.C.A.R.E.S. framework, such as Altruism, was coded. A competency item could be attributed to more than one aspect of I.E.C.A.R.E.S. All items, humanistic and not, were coded by the CanMEDS framework as one of the seven CanMEDS competency domains of Medical Expert, Communicator, Collaborator, Leader, Scholar, Professional or Health Advocate. ${ }^{18} \mathrm{~A}$ competency item could be attributed to more than one CanMEDS domain.

To determine the level of agreement between coders the Kappa statistic and percent agreement were performed. The kappa statistic for humanism and non-humanism coding was 0.92 and the percent agreement was $99 \%$ between the two reviewers. Descriptive statistics were used to describe the proportion of each curricula which addressed humanistic competencies, the nature of the humanistic competencies and the proportion of each CanMEDS competency item.

\section{Results}

\section{To what extent are humanistic competencies included?}

From the 17 curricula 7792 curricular items were identified. 780 of 7792 items (10\%) were identified as humanistic and 7012 (90\%) were identified as non-humanistic. The proportion of humanistic items ranged from $2 \%$ to $26 \%$ in individual curricula. Twelve of seventeen curricula had $<10 \%$ of items attributed to humanism. The proportion of humanistic items has been increasing from the curricula published in 1980-1989 (3\%) to the curricula published in 2010-2017 with a mean of $11 \%$ (4 to $25 \%$ ) (Table II).

\section{What is the nature of the humanistic competencies?}

Of the 780 humanistic items, 886 were aligned with the I.E.C.A.R.E.S. framework. Of the 886, 48 (5\%) were under Integrity, 18 (2\%) under Excellence, 272 (31\%) under Compassion, 12 (1\%) under Altruism, 311 (35\%) under Respect, 212 (24\%) under Empathy and 13 (1\%) under Service (Table III). Examples of competency items in each of the I.E.C.A.R.E.S domains are provided in Table IV. 
Table II: Proportion Humanism by Year and Region

\begin{tabular}{ll}
\hline Publication Year & $\begin{array}{l}\text { Proportion Humanism } \\
\text { Mean (Range) }\end{array}$ \\
\hline $1980-1989$ & $3 \%(\mathrm{~N} / \mathrm{A})$ \\
\hline $1990-1999$ & - \\
\hline $2000-2009$ & $5 \%(2-7 \%)$ \\
\hline $2010-2017$ & $11 \%(4-25 \%)$ \\
\hline Region of Publication & \\
\hline Africa & - \\
\hline Asia & - \\
\hline Oceania & $10 \%(5-15 \%)$ \\
\hline Europe & $9 \%(2-26 \%)$ \\
\hline Latin Americas & - \\
\hline North America & $5 \%(3-6 \%)$ \\
\hline
\end{tabular}

Table III: Distribution of Humanistic Competencies within the I.E.C.A.R.E.S Framework

\begin{tabular}{llllllll}
\hline \multicolumn{7}{c}{ Number of Items per IECARES Category } \\
\cline { 2 - 8 } & Integrity & Excellence & Compassion & Altruism & Respect & Empathy & Service \\
\hline Humanistic & $48(5 \%)$ & $18(2 \%)$ & $272(31 \%)$ & $12(1 \%)$ & $311(35 \%)$ & $212(24 \%)$ & $13(1 \%)$ \\
\hline
\end{tabular}

Table IV: Examples of Competency Items for each Aspect of the I.E.C.A.R.E.S Framework

\begin{tabular}{|c|c|}
\hline $\begin{array}{l}\text { Humanism } \\
\text { Domain }\end{array}$ & Example Competency Item \\
\hline Integrity & $\begin{array}{l}\text { "Practice medicine in accordance with medical ethics and patient rights"20 } \\
\text { "The surgical oncologist must take responsibility for their actions and outcomes with hones- } \\
\text { ty and a desire to continually improve, always putting the patient's needs first"21 }\end{array}$ \\
\hline Excellence & $\begin{array}{l}\text { "Surgical oncologists have a professional duty to maintain and continually update their } \\
\text { expertise to enable them to offer patient care that maximizes beneficial outcomes within the } \\
\text { limits of the healthcare environment in which they practice" } 21\end{array}$ \\
\hline $\begin{array}{l}\text { Compassion \& } \\
\text { Collaboration }\end{array}$ & $\begin{array}{l}\text { "Ability to elicit the patient's wishes with regard to the aims of treatment and to give the } \\
\text { treatment alone or in collaboration with other specialists"22 } \\
\text { "Listening to patients and responding to their questions, concerns and preferences and } \\
\text { keeping them informed about the progress of their care"23 }\end{array}$ \\
\hline Altruism & $\begin{array}{l}\text { "The surgical oncologist must take responsibility for their actions and outcomes with hones- } \\
\text { ty and a desire to continually improve, always putting the patient's needs first" }\end{array}$ \\
\hline $\begin{array}{l}\text { Respect \& } \\
\text { Resilience }\end{array}$ & $\begin{array}{l}\text { "Always considerate, polite and thoughtful of patients and colleagues"24 } \\
\text { "Ability to elicit the patient's wishes with regard to the aims of treatment and to give the } \\
\text { treatment alone or in collaboration with other specialists"22 }\end{array}$ \\
\hline Empathy & $\begin{array}{l}\text { "Recognizes the impact of bad news on the patient, carers, staff members and self" } 23 \\
\text { "Depression and anxiety, the role of the clinical nurse specialist. How to recognise the } \\
\text { symptoms and signs of psychological distress and secondary mental illness. Management } \\
\text { strategies"25 }\end{array}$ \\
\hline Service & $\begin{array}{l}\text { "Accepts additional duties in situations of unavoidable and unpredictable absence of collea- } \\
\text { gues ensuring that the best interests of the patient are paramount"23 }\end{array}$ \\
\hline
\end{tabular}




\section{What is the relationship of humanism to non-medical expert competencies?}

Of the 8023 CanMEDS attributions 5549 (69\%) were Medical Expert, 685 (9\%) were Communicator, 391 (5\%) were Collaborator, 267 (3\%) were Leader, 528 (7\%) were Scholar, $518(6 \%)$ were Professional and 85 (1\%) were Health Advocate. The majority of the humanistic items were in the Professional ( $n=261,35 \%)$, Medical Expert $(n=232,31 \%)$ or Communicator ( $n=190,25 \%)$ CanMEDS domains. See Figure I.

A

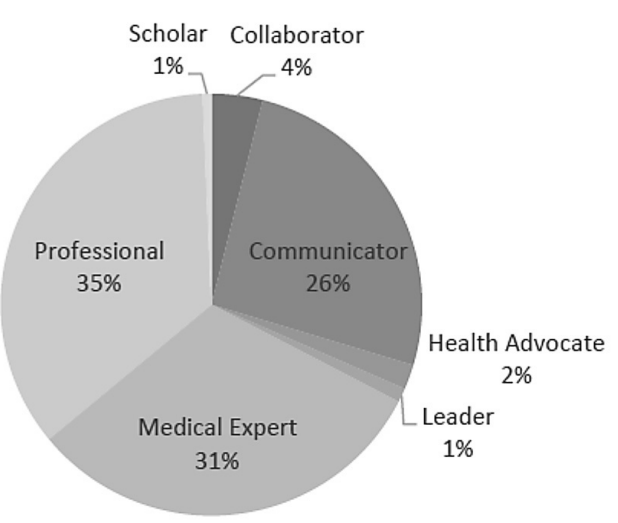

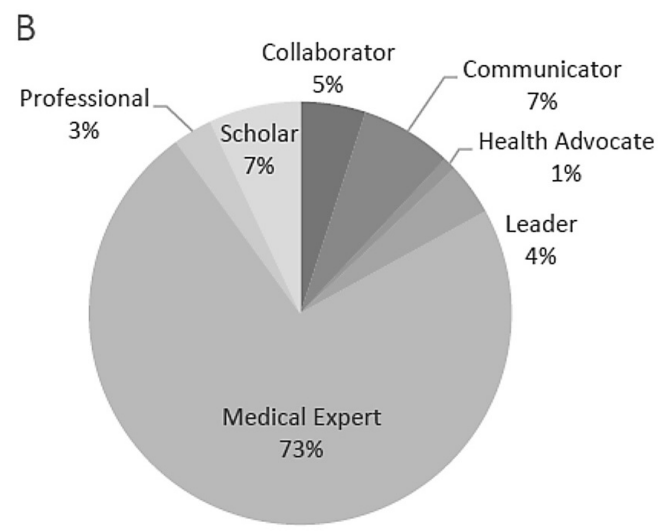

Figure I: Proportion of Humanistic (A) and Non-Humanistic (B) Competency Items by CanMEDs role

\section{Discussion}

Our analysis of global oncology curricula has shown that humanistic competencies comprise a wide range, $2 \%$ to $26 \%$, of curricular content. While the proportion of humanism has increased over time, with a greater proportion of humanism represented in more recently published curricula, the majority have less than $10 \%$ of the curricula composed of humanistic competencies. While there is no consensus on the ideal proportion of curricula that should reflect humanistic competencies, these skills are perceived as important conditions for improving the quality of care received by patients, realizing shareddecision making with patients and addressing systems level issues. ${ }^{8,26}$ As such, devoting less than $10 \%$ of curricula to humanistic competencies could be problematic. Defining and describing curricula to meet the wide breadth of competencies while accounting for and appreciating regional and cultural differences is a challenge. This is especially true when curricular planning is intended to have global application. However, as efforts continue to revise, update and improve global curricula there is a need to reflect on the risk of reductionism in the definition of competencies and to ensure humanistic concepts that are conducive to supporting local needs of patients are preserved. ${ }^{27}$ The integration of humanistic competencies into global oncology curricula requires advocates for these skills. Educators have been successful in implementing humanism in medical curricula by creating a sense of urgency. ${ }^{28}$ Our data may assist in articulating this imperative platform for oncology by providing a description of the current, highly variable, state of humanism in global oncology curricula. 
It has been previously noted by Martimianakis et al that the majority of publications addressing humanism in medical education originated from a North American context and there exists a conflation between humanistic competencies and professionalism. ${ }^{2}$ Our analysis supports this finding, with the highest proportion of humanistic items (35\%) attributed to the professionalism domain. In the past two decades there has indeed been a growing focus on including non-medical expert competencies, such as professionalism, into training frameworks in Western contexts. ${ }^{18}$ While we are not able to ascertain why this conflation between humanism and professionalism exists, it is possible that the focus on directing and shaping the behavior of individuals to conform to regulatory and professional norms supersedes other aspects of care such as Empathy and Service. In the Western setting the majority of medical schools have made efforts to incorporate humanism into medical training and this has been operationalized through its link to Professionalism. ${ }^{29}$ Health Advocate represents $2 \%$ of humanistic items in existing curricula. The lack of focus on Health Advocate which emerged in our analysis, is surprising given the global lack of access to cancer care and the recognized need for addressing health inequities in access to treatment. One possible challenge in implementing humanistic curricula more comprehensively could include a lack of shared understanding of complex concepts such as Empathy. In addition, where measurement for assessment is important, a lack of clear understanding of what to measure in humanistic competencies represents a potential barrier. ${ }^{30}$ The most commonly used method for assessment of humanistic competencies involves self-report and most of the reports in the literature originate from North America. ${ }^{31}$ In addition, Professionalism is the dimension which has received the most attention regarding its assessment, and this may be a contributing factor to the conflation between humanism and professionalism. Future efforts to more comprehensively include the diverse aspects of humanism within oncology curricula can be assisted by understanding the current state of existing curricula reported in this paper. Our data has showed that the representation of humanism in existing curricula is focused on Respect, Compassion and Empathy and that there is a conflation between humanism and Professionalism and a relative paucity with Health Advocate.

The cultural influence of Western ideology has been reported for humanistic competencies in medicine. ${ }^{10}$ As such, there may exist a mismatch between aWestern concept of humanism and its suitability in non-Western domains, ${ }^{32}$ creating a potential barrier to integrate health care and delivery practices associated with humanism into global curricula. The known differences in the East-West healthcare ecosystems for cancer care adds to the complexity of this discussion. ${ }^{33}$ There is a lack of literature on how applicable Western frameworks of humanism are globally. ${ }^{12}$ Cultural diversity and contextual factors limit the ability to directly transfer Western pedagogical approaches and priorities to non-Western settings. ${ }^{34}$ However, local contextualization of Western approaches has been achieved albeit with significant effort and time dedicated to achieving this. A Chinese research 
group has shown that using Nominal Group Technique it is feasible to contextualize and locally apply Western frameworks in a Chinese setting. ${ }^{10} \mathrm{~A}$ Taiwan group has employed the six-step curriculum development framework as a method to integrate local cultural and societal needs into Western framed humanistic profiles..$^{35}$ One driving factor for the integration of humanistic competencies in non-Western settings can be the objective to meet international accreditation standards. ${ }^{36}$ Pan et al have demonstrated both cultural influence as well as conflict with Western ideologies in East Asian settings. ${ }^{14}$ The cultural basis of humanistic competencies has necessitated a call for a global approach to integrate these competencies. ${ }^{17,37}$ Skills in humanistic domains extend beyond an empathetic, caring relationship with patients and families and involve the recognition and ability to navigate differences in values, and to understand the impact of power relationships in healthcare. ${ }^{27}$ Although the need for humanism in healthcare transcends culture, further work is needed to understand the barriers to inclusion of greater humanistic competencies in global curricula.

There are several limitations with this work. We have applied two specific frameworks, CanMEDS and I.E.C.A.R.E.S, to perform this analysis, while we acknowledge that other frameworks of medical competency and conceptualizations of humanism exist. Moreover, the two frameworks have a Western orientation. However, the curricula which formed the basis of this analysis were largely developed by Western authors. In addition, at this time we are not aware of a non-Western framework which addresses humanism. However, if one were available, an analysis comparing the application of Western and non-Western frameworks would provide essential information regarding the degree of relevance of current global curricular frameworks which rely on Western perspectives. These data can serve as a basis for critically reviewing the content of future oncology curricula. In addition, the language of competency items is negotiated by members of the curriculum development group and reflect their sociocultural orientation and biases. ${ }^{38}$ Therefore, individual competency items are open to interpretation by individuals with diverse socio-geographic orientations and we cannot ascertain the degree of variability in the interpretation of these global oncology curricular items. Any differences in this respect may be best elucidated by a qualitative approach.

\section{Conclusion}

The proportion of humanistic competencies has been increasing in global oncology curricula over time, however the overall proportion remains low and represents a largely Western perspective on what constitutes humanism in healthcare. The representation of humanism primarily focuses on the I.E.C.A.R.E.S attributes of Respect, Compassion and Empathy. Future global curricular efforts may benefit from attention to incorporating all aspects of humanistic competencies. Further work is needed to understand how humanism may be perceived differently in different cultural and geographic contexts. 


\section{References}

1. Thibault GE. Humanism in Medicine: What Does It Mean and Why Is It More Important Than Ever? Acad Med 2019 Aug; 94(8): 1074-1077.

2. Martimianakis MA, Michalec B, Lam J, Cartmill C, Taylor JS, Hafferty FW. Humanism, the hidden curriculum, and educational reform: a scoping review and thematic analysis. Acad Med 2015;90. Suppl 11:S5-S13.

3. Arnold P. Gold Foundation. FAQS $>$ What is Humanism in Healthcare [Web page]. Englewood Cliffs, NJ: Arnold P. Gold Foundation; 2018. [Available at: https://www.gold-foundation.org/ about-us/faqs/; cited May 1, 2018]

4. Miller SZ, Schmidt HJ. The habit of humanism: a framework for making humanistic care a reflexive clinical skill. Acad Med 1999;74(7):800-803.

5. Whitehead C. Scientist or science-stuffed? Discourses of science in North American medical education. Med Educ 2013;47(1):26-32. https://doi.org/10.1111/j.1365-2923.2011.04136.x

6. Hartzband $\mathrm{P}$, Groopman J. Keeping the patient in the equation-humanism and health care reform. New Engl J Med 2009;361 (6):554-555

7. Robbins JA, Bertakis KD, Helms LJ, Azari R, Callahan EJ, Creten DA. The influence of physician practice behaviors on patient satisfaction. Fam Med 1993;25(1):17-20.

8. Frenk J, Chen L, Bhutta ZA, Cohen J, Crisp N, Evans T, Fineberg H, Garcia P, Ke Y, Kelley P et al. Health professionals for a new century: transforming education to strengthen health systems in an interdependent world. Lancet 2010;376(9756):1923-1958. https://doi.org/10.1016/ S0140-6736(10)61854-5

9. Shanafelt TD, Gradishar WJ, Kosty M, Satele D, Chew H, Horn L, Clark B, Hanley AE, Chu Q, Pippen J et al. Burnout and career satisfaction among US oncologists. J Clin Oncol 2014;32(7):678686. https://doi.org/10.1200/JCO.2013.51.8480

10. Horowitz CR, Suchman AL, Branch WT, Jr., Frankel RM. What do doctors find meaningful about their work? Ann Internal Med 2003;138(9):772-775.

11. Kumagai AK. Beyond "Dr. Feel-Good": A Role for the Humanities in Medical Education Acad Med 2017;92(12):1659-1660.

12. Gosselin K, Norris JL, Ho MJ. Beyond homogenization discourse: Reconsidering the cultural consequences of globalized medical education. Med Teach 2016;38(7):691-699.

13. Al-Eraky MM, Chandratilake M. How medical professionalism is conceptualised in Arabian context: a validation study. Med Teach 2012;34 Suppl 1:S90-95.

14. Pan H, Norris JL, Liang YS, Li JN, Ho MJ. Building a professionalism framework for healthcare providers in China: a nominal group technique study. Med Teach 2013;35(10):e1531-1536.

15. Sefton AJ. New approaches to medical education: an international perspective. Med Prin Pract 2004;13(5):239-248.

16. Ho MJ, Abbas J, Ahn D, Lai CW, Nara N, Shaw K. The "Glocalization" of Medical School Accreditation: Case Studies From Taiwan, South Korea, and Japan. Acad Med 2017;(12):1715-1722.

17. Jha V, McLean M, Gibbs TJ, Sandars J. Medical professionalism across cultures: a challenge for medicine and medical education. Med Teach 2015;37(1):74-80. 
18. Frank JR, Snell L, Sherbino J, eds. CanMEDS 2015 Physician Competency Framework. Ottawa, ON: Royal College of Physicians and Surgeons of Canada; 2015.

19. NVivo qualitative data analysis software; QSR International Pty Ltd. Version 11; 2015.

20. Coffey M, Mullaney L, Bojen A, Vaandering A, Vandevelde G. 2012. Recommended ESTRO Core Curriculum for Radiation Oncologists/Radiotherapists, 3rd edition.asd

21. Are C, Berman RS, Wyld L, Cummings C, Lecoq C, Audisio RA. Global curriculum in surgical oncology. Eur J Surg Oncol 2016;42(6):754-766. https://doi.org/10.1245/s10434-016-5239-7

22. Dittrich C, Kosty M, Jezdic S, Pyle D, Berardi R, Bergh J, El-Saghir N, Lotz JP, Osterlund P, Pavlidis $\mathrm{N}$ et al. ESMO / ASCO Recommendations for a Global Curriculum in Medical Oncology Edition 2016. ESMO Open 2016 Sep 29;1(5):e000097. https://doi.org/10.1136/esmoopen-2016-000097

23. Faculty of Clinical Oncology. Specialty Training Curriculum for Clinical Oncology. London: Royal College of Radiologists, https://www.rcr.ac.uk/sites/default/files/docs/oncology/pdf/ Curriculum\%20-\%20Clinical\%20Oncology\%20-\%20FINAL\%20-\%20110610.pdf; 2016 [accessed May 14, 2019].

24. Joint Royal Colleges of Physicians Training Board. Speciality training curriculum for medical oncology. London: Joint Royal Colleges of Physicians Training Board, https://www.jrcptb.org. uk/sites/default/files/2017\%20Medical\%200ncology\%20Curriculum\%20FINAL.pdf; 2017 [accessed May 14, 2019].

25. ESSO Curriculum Committee. ESSO Core Curriculum 2013. Eur J Surg Oncol 2013 Sep 27; 39(Suppl 1):S1-S31. https://doi.org/10.1016/j.ejso.2013.07.084

26. Horlait M, Leys M, De Greve J, Van Belle S. Integrating communication as a core skill in the global curriculum for medical oncology. Ann Oncol 2017;28(3):670-671.

27. Gaufberg E, Hodges B. Humanism, compassion and the call to caring. Med Educ 2016;50(3):264-266.

28. Steinert Y, Cruess RL, Cruess SR, Boudreau JD, Fuks A. Faculty development as an instrument of change: a case study on teaching professionalism. Acad Med 2007;82(11):1057-1064.

29. Montgomery L, Loue S, Stange KC. Linking the Heart and the Head: Humanism and Professionalism in Medical Education and Practice. Fam Med 2017; 49(5):378-383.

30. Holmboe E. Bench to bedside: medical humanities education and assessment as a translational challenge. Med Educ 2016;50(3):275-278.

31. Buck E, Holden M, Szauter K. A methodological review of the assessment of humanism in medical students. Acad Med 2015;90 Suppl 11:S14-23.

32. Ho MJ, Yu KH, Hirsh D, Huang TS, Yang PC. Does one size fit all? Building a framework for medical professionalism. Acad Med 2011;86(11):1407-1414.

33. Agarwal JP, Lee VH-F. East Meet West: Convergence of the Art and Science of Oncology. Clinical Oncology 2019; 31:487-489

34. Frambach JM, Driessen EW, Chan LC, van der Vleuten CP. Rethinking the globalisation of problem-based learning: how culture challenges self-directed learning. Med Educ 2012;46(8):738747. 
35. Tsai SL, Ho MJ, Hirsh D, Kern DE. Defiance, compliance, or alliance? How we developed a medical professionalism curriculum that deliberately connects to cultural context. Med Teach 2012;34(8):614-617.

36. Chiu CH, Arrigo LG, Tsai D. Historical context for the growth of medical professionalism and curriculum reform in Taiwan. Kaohsiung J Med Sci 2009;25(9):510-514.

37. Al-Rumayyan A, Van Mook W, Magzoub ME, Al-Eraky MM, Ferwana M, Khan MA, Dolmans D. Medical professionalism frameworks across non-Western cultures: A narrative overview. Med Teach 2017;39 Suppl1:S8-S14.

38. Lurie SJ, Mooney CJ, Lyness JM. Commentary: pitfalls in assessment of competency-based educational objectives. Acad Med 2011;86(4):412-414. 
CHAPTER 5

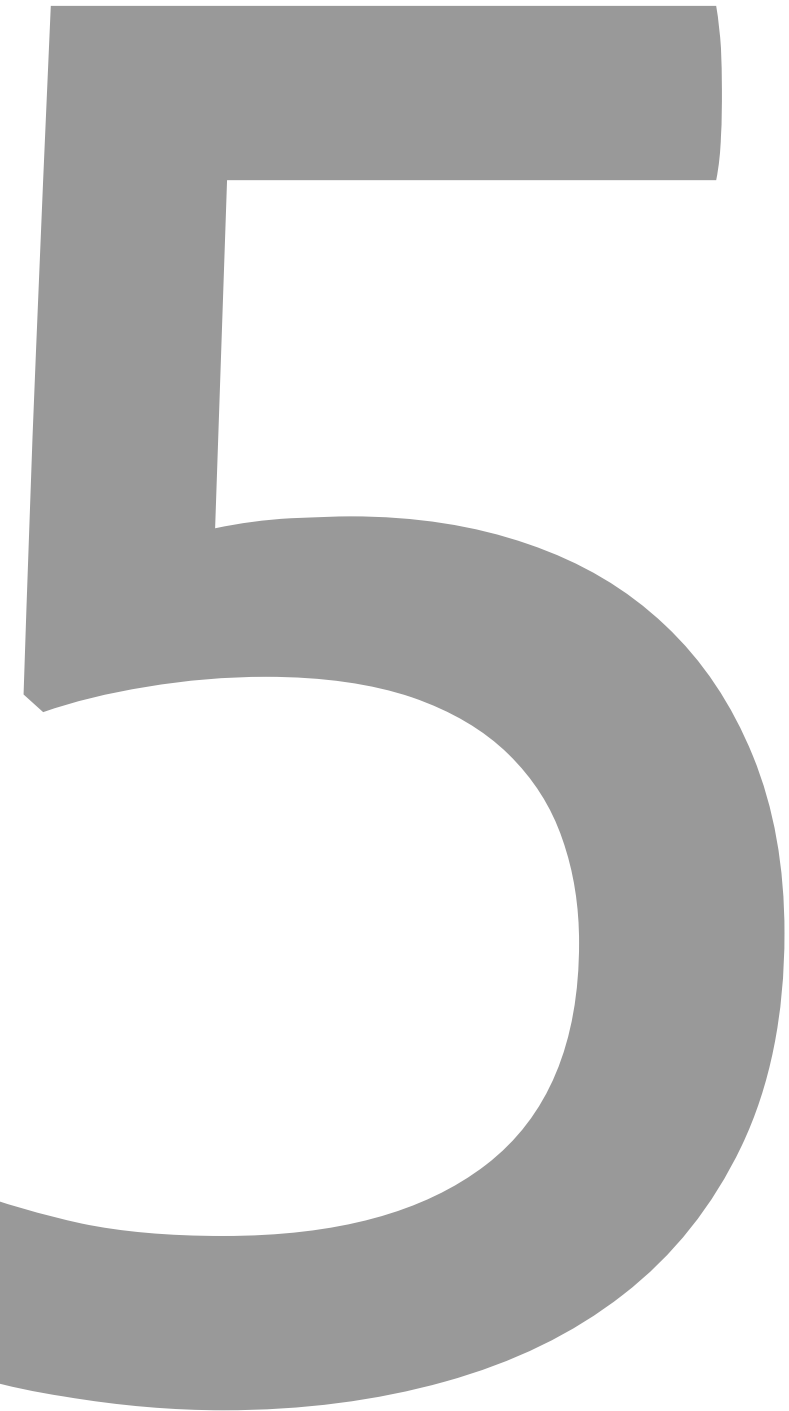




\section{Exploring Glocalization in the Construction and Implementation of Global Curricula}

Published as: Giuliani M, Frambach J, Driessen E, Martimianakis M. Exploring Glocalization in the Construction and Implementation of Global Curricula. Journal of Cancer Education. 2020 


\section{Background}

Despite proposed advantages of global curricular harmonization including physician mobility and improving the quality of care the challenges and unintended consequences require greater study. The aim of this study was to problematize the concept and implementation of global oncology curricula and their relationship to local contexts of power and culture.

\section{Methods}

Fourteen international participants involved in the development and implementation of global oncology curricula completed in-depth, one-on-one semi-structured interviews lasting 40-60 minutes. Snowball sampling was employed. Through iterative analyses, using an abductive approach, the study team discussed and reviewed the data and made revisions through collaborative analysis to enhance comprehensiveness and to improve credibility. In the final analysis the meaning and implication of the themes were discussed yielding a conceptual analysis.

\section{Results}

Our data have articulated 5 key challenges for global curricula including 1) Ambiguous or conflicting perspectives on the purpose and scope of Global Oncology Curricula 2) Insufficient representation of diverse perspectives and realities in the creation of the final curricula 3) A rigid conceptualization of competency requirements 4) A mismatch between the curricular requirements and local context and 5) The influence of power relationships and decision makers. Leveraging the strengths of diversity including fostering representation, addressing power differentials and factoring local contexts may be an approach to mitigating these challenges.

\section{Conclusions}

Global oncology curricula may serve important advocacy roles within the healthcare system. Leveraging diversity may positively impact the common challenges in the construction and implementation of global oncology curricula. 


\section{Introduction}

There is a growing health human resource crisis in cancer care. Training programs globally are working to address the need for changes and increase capacity in oncology training ${ }^{1}$. In addition, there is a perceived mismatch between the training curricula for healthcare professionals and the needs of patients, families and the health-care system argued to fuel a healthcare crisis ${ }^{2}$. Given the global nature of this crises, international organizations have focused on developing harmonized training standards or global curricula ${ }^{3,4}$. These efforts to produce curricula with a global focus ${ }^{5}$ raise the question of how the process for developing global oncology curricula is aligned with their goals.

There are several common motives for creating global curricula including defining specialty specific standards, harmonizing training standards and improving the quality of training ${ }^{6}$. Despite many proposed advantages of global curricular harmonization efforts including physician mobility and improving the quality of care ${ }^{7}$ the challenges and unintended consequences, including a mismatch between global priorities and local needs ${ }^{8}$, requires greater study ${ }^{9}$. We have previously reported, based on a scoping review, that intercountry variation, the need for a multi-stakeholder approach, and complexity in implementation are important aspects related to global medical curricula ${ }^{6}$. The potential unintended effects of engaging in this form of global standardization, such as perpetuating the Western medical priorities (for example biomedicine ${ }^{10}$ ) requires further study. The concept of glocalization is relevant in this discourse on global medical curricula because it refers, in medical education, to developing the skills and knowledge to address the universal and particular or to be adaptable in the global and local spaces ${ }^{11}$. What is greatly lacking in the global-local debate in the medical education literature is evidence of positive or negative impact of global curricula on clinical care, the healthcare system, and trainees' knowledge and skills ${ }^{8,12}$. A first step in understanding impact is to understand the factors impacting implementation.

The existing literature is largely from the Western perspective ${ }^{5}$, and as such we are not able to ascertain the degree of local customizations that occurs or the degree to which these curricula address local health care needs. The majority of global curricula are constructed through social group processes such as committees, task forces or a Delphi process ${ }^{6}$. These approaches, while practical, do not necessarily preserve nor promote diversity especially for minority expert voices. Any social process involving individuals is susceptible to biases including conflicts of interest and political pressures ${ }^{6}$. How these biases impact an already challenging situation to establish what is 'core' curricula coupled with the tension between meeting local needs and achieving international standards ${ }^{13}$ requires further investigation. 
The aim of this study was to problematize the concept and implementation of a global oncology curricula in postgraduate training. We explored, through interviews with international participants, how global oncology curricula are constructed and implemented and their relationship to local contexts of power and culture.

\section{Methods}

\section{Design and procedures}

This qualitative study involved interviews with international participants. Study recruitment began with individuals involved in the development of global oncology curricula based on publication or professional reputation. Participants were selected who have experience and/or expertise in the area of global oncology education in the form of curriculum development or local implementation or translation to practice and included representatives of organizations with global curricula as well as educators in diverse geographic areas around the world. We believed this approach and these participants would yield rich data and enable us to further understand the construction and implementation of global oncology curricula. To address the dominance of Western perspectives we specifically targeted participants with non-Western experiences or lowmiddle income country perspectives. Snowball sampling was employed for this study ${ }^{14}$.

A semi-structured interview guide was developed and was adapted during the data collection process based on data from prior interviews. Interview participants were asked to reflect on the concept of a global curriculum, the purpose it serves for training oncologists, their perception of differences from local or traditional curricula/ pedagogical practices and perceived advantages and limitations of such a concept. Interviewees also reflected on their perceptions of how global curricula may be perceived within their local contexts with specific focus on power, culture and pedagogical priorities. All interviews took place in English however the option of utilizing a translator was available. The interviews lasted 40 to 60 minutes. The interviews were conducted in person, over the phone or through electronic interfaces such as Skype ${ }^{T M}$. This facilitated the participation of global experts who reside in geographically disparate locations. The interviews were audiotaped and transcribed verbatim. University Health Network research ethics board exemption was received for this study [waiver 19-0310]. All participants provided informed written consent to participate. To ensure anonymity participant quotes are reported by participant number.

\section{Participants and Sampling}

Fourteen participants completed in-depth, one-on-one semi-structured interviews with a study investigator (MG). Data collection occurred from February to May 2019. Sampling continued until there was sufficient information power to address the dominant themes 
15. The participant sample was representative of different geographic regions, genders and professional scopes of practice to ensure diverse perspectives were sought. Of the 14 participants 7 (50\%) were female. Five (36\%) were from Europe, $3(21 \%)$ were from Africa and 3 (21\%) from Asia and 1 (7\%) participant each from North America, Latin America and Oceania. The participants were clinical or medical oncologists $(n=6)$ or radiation medicine professionals $(n=8)$. Some participants carried double roles with experience in diverse geographic healthcare settings and/or experience across different oncology professional scopes of practice.

\section{Analysis}

One study investigator (MG) read each transcript and performed an initial analysis to produce a coding framework ${ }^{16}$. Other investigators (TM, ED and JF) reviewed and coded a subset of the transcripts. The analysis built upon previous work by the study team in the area of global curriculum development ${ }^{5-6,17}$. The framework was then used to code the transcripts. Through iterative analyses, using an abductive approach, the study team discussed and reviewed these data and made revisions through collaborative analysis to enhance comprehensiveness and to improve credibility ${ }^{18}$. The abductive approach included applying a combination of theoretically derived codes and capturing emerging codes. In the final analysis the meaning and implication of the themes were discussed yielding a conceptual analysis. NVivo version 11 software was used to facilitate coding and analysis ${ }^{19}$.

\section{Research Team and Reflexivity}

The research team consisted of an oncology physician with experience in curriculum design with an international focus and education scientists whose expertise is on globalization and internationalization in medical education. Members of the research team have also served as educational advisors for organizations which publish global curricula. The research team explicitly discussed the impact their prior experience and professional training had on the conduct of the study and interpretation of the data.

\section{Results}

A number of challenges were identified in the construction and implementation of Global Oncology Curricula. These challenges include 1) Ambiguous or conflicting perspectives on the purpose and scope of Global Oncology Curricula 2) Insufficient representation of diverse perspectives and realities in the creation of the final curricula 3) A rigid conceptualization of competency requirements 4) A mismatch between the curricular requirements and local context and 5) The influence of power relationships and decision makers. First we elaborate on these challenges, then we introduce how diversity can be leveraged to potentially mitigate these challenges (Table 1). 
1. Ambiguous or conflicting perspectives on the purpose and scope of Global Oncology Curricula:

All participants problematized the concept of a "global curriculum". Specifically, they all identified the term "global" as complex and a common definition or understanding of how the label 'global' was applied to a curriculum was not articulated. Global curricula as a construct was identified by some as positive and linked to aspirational view points as well as an opportunity for consensus building and by others as negative being a vehicle for imposing and perpetuating a dominant perspective. The positive and negative tensions around global curricula were captured as "I both like the term and I hate the term. I like the term because it's easy to understand what this is about. But this is also why I hate it because for me global also sounds a bit imperialistic and whatever we do in teaching education in trying to raise the standards it should not be imperialistic. So, in that sense I really do like and I always know this will never be global but it would be something which would hopefully be applicable across the globe." (P014)

The concept of 'curriculum' was also problematized. After reflecting on the concept of 'global' P003 went on to state "the other part which is open to interpretation is what's meant by curriculum...A curriculum should mean a lot more than that [competencies or learning outcomes] in terms of some kind of structure to a training program to ensure that the learning opportunities and experiences and teaching that is required is present to support attaining those competencies and also that there be some form of matching or complementary assessment". These diverse view points about the scope of the components of the curricula under the heading of curriculum is important when one reflects on the implementation complexities and whether some curricula may not include these other aspects due to the global local tensions and complexities. This problematization of the concept of curricula also lends itself to the discourse on the purpose of these curricula. This leads us to the next challenge around representation of diverse perspectives.

2. Insufficient representation of diverse perspectives and realities in the creation of the final curricula:

According to the participants a lack of representation, particularly of low- and middleincome countries (LMIC), in development was directly linked to a challenge of implementing curricula globally. A core development challenge is "how best to make them [global curricula] useful in environments that may not have had much input into the development" (P003).

The participants identified the process of determining the content of the curricula as challenging. The presence of "different confrontation styles and different conflict resolution styles depending on a different culture" (P002) was one aspect of this challenge. Most curricula are created through a social group process [6] and as such differences in 
communication and conflict resolution styles may directly impact whose perspective is more dominant and thus reflected to a greater degree in the final product. The degree to which differences of opinion or perspectives were acknowledged and/or resolved in the development process is unknown. Other participants described a co-creation approach for "global" curricula. When discussing the construction of these curricula using a collaborative approach they described the processes of glocalization where the universal is negotiated with the local to produce a final produce. A participant speaking from a European perspective about curricular co-creation stated "we were very conscious that we did not want to impose what we thought everyone should know from a [European] viewpoint in [other countries]" (P008). Buy-in from stakeholders was also seen as critical for implementation; "If you don't get buy in and you're just given a set of core competencies that you may think are old fashioned or not relevant to your particular culture, then you're not going to adhere to them as strongly as if you've actually bought into it" (P008).

\section{A rigid conceptualization of competency requirements:}

The concept of what constitutes competence for completion of postgraduate training, leading to independent practice, was identified as an area of controversy. This diversity in where the bench mark for competence is set or even what is understood by the term 'competence' was presented by the participants as a challenge. "I think these local sort of hurdles where you set the bar for saying you completed your training even though you never really complete your training... where you set the bar for competence is going to change between different countries." (P008). Varying scopes of practice and duration of training prior to independent practice between countries was a specific area contributing to this controversy related to competence. "What a doctor does in one place may be different to another place" and went on to state "you have this mismatch [globally] that you've got some specialties that are very country specific and you've got other specialities which are more general" (P008). One participant when describing the challenges within a region stated (P014) "it's still 28 to 30 different countries with totally different systems and in many ways we have organized our health system, in the way we have organized our education in terms of economically wealth."

Participants drew links to the greater health systems context in relation to the concept of competence and curricular content. One participant from Africa saw an association between the concepts and tensions of human resource requirements and training considerations. This participant reflected that a curriculum in clinical oncology and ultimately competence as a clinical oncologist would be most relevant to address the health human resource needs in their context "trained as a clinical oncologist because in this part of the world you just can't afford to have separate radiation oncologist and separate medical oncologist" (P010). The clinical oncologist discourse is also relevant in other regions including Europe ${ }^{20}$. 
Participants go on to postulate that to given the variation in where the bar for competence for independent practice is set in different jurisdictions global curricula should be adaptable. The curricula itself is considered by some as a guiding document "so the adaptation is done locally and the curricula is more of an overall recommendations document" (P004). Adaptation here was conceptualized as the global curricula serving as an overall repository where local educators could then accept, reject or change content to fit their purposes and needs for where they define training priorities and competence. There was an articulated tension between the comprehensiveness or size of the curricula and the possibility for flexibility for local priorities. "I think as it [the curricula] does get larger and more sophisticated it gets harder for it to be adopted" (P005). The conceptualization of what a sophisticated curriculum entails was not clear from the interviews and could possibly reflect a perception of educational infrastructure or clinical infrastructure. This concept of adaptability leads into the next challenge which is related to implementation and local context.

4. A mismatch between the curricular requirements and local context:

Reflection by national stakeholders about whether or not to implement a global curricular document was related to whether or not they felt they could meet the requirements set out in it. There were two main facets considered by participants when deciding how applicable a curriculum is. These included the length of training and the medical or systems factors.

There are many factors which determine the length of training in a speciality including historical aspects, clinical service demands and international recommendations. The need for clinical service was a critical factor in determining the length of training as articulated by our participants. They discussed a relation between increasing needs for clinical services and keeping the length of training short in areas with the greatest need for health human resources; "on the one hand they want to make it a five year program but on the other hand... there's an alarming lack of colleagues in the country" (P011) . Therefore, during the construction of global curricula, consideration of these local realities and discussion around where the curricula can be modified and to what extent may assist in endorsement and implementation.

The medical or systems factors related to local availability of medication, such as certain chemotherapy regimens or certain technologies such as image guided radiotherapy.

Participant 006 stated "I want my residents to know all the new stuff that's happening there even if we don't have access to it... for two reasons. First is it good enough for us to adopt or advocate for the government to pay especially for our universal health coverage or the patient has private insurance and also to explain to the patients as they are very well informed...so it has to do with stewardship of resources. ... recently they [the residents] 
were presenting an American trial for lung cancer chemotherapy and they know that it might not have for many years to come but they do want to know about it so then if it becomes available for all the patients. It's always a negotiation between the evidence that [is] usually generated in high income countries and the local environment. So, for example, the [breast cancer] patient may not be tested for estrogen receptor because it's not available but because, they have done some health services research and they go for some of the evidence in East Africa, maybe there's more estrogen receptor positive cancers they could treat empirically." Consideration of these systems level factors is also related to decision makers and those in positions of power. This leads us to our final challenge.

5. The influence of power relationships and decision makers:

Power relationships were critical to implementation of global curricula with respect to ensuring decision makers, such as government or universities, possessed the required insight into the medical reality of their local context to advocate for and approve the necessary supports for curricular implementation. Having a mutual understanding with decision makers is critical because "the ministry is responsible for developing so-called standards... professional standards and those examinations that one needs to pass in order to get a certificate of consulting"(P011). One participant (P009) describing their experience with barriers to implementing core curricula across different European countries reflected that "this kind of lack of understanding or lack of awareness of government level that this [curriculum] is actually required" is a key barrier to get things implemented. They went on to elaborate that "Buy-in from who I would call the primary stakeholders so they are actually the policymakers whether that's at the national level at the Ministry of Health ... [or at] a local level at the University or the clinic" (P009) is crucial. P004 reflects on the use of global curricula as advocacy tools with decision makers stating "I'll focus more on what is expected [by] someone with regulatory power instead of how it [the curricula] can be delivered". An example to illustrate this point is that if the purpose of the global curricula is to advocate for longer periods of training there should be recommendations on minimum length of training as found in some curricula ${ }^{21,22}$ whereas this may be omitted or not emphasized if the purpose is to promote homogeneity in clinical skills. A collaborative, international approach to curriculum design was seen as a powerful mechanism to advocate for training needs to decision makers in local jurisdictions.

\section{Leveraging Diversity}

While our participants identified these five challenges which have been described about they also identified how diversity was a critical factor in mitigating these challenges. Table 1 articulates the way in which diversity can be leveraged to address these challenges in Global Oncology Curriculum construction and implementation. 
Table 1: How Leveraging Diversity could positively Influence Global Oncology Curricula

\begin{tabular}{|c|c|c|c|}
\hline Challenges & $\begin{array}{l}\text { Leveraging the } \\
\text { strengths of } \\
\text { diversity.... }\end{array}$ & How? & Illustrative quotes \\
\hline $\begin{array}{l}\text { Ambiguous or con- } \\
\text { flicting perspectives } \\
\text { on the purpose and } \\
\text { scope of Global Onco- } \\
\text { logy Curricula }\end{array}$ & $\begin{array}{l}\text { Requires clarifying } \\
\text { the curricular } \\
\text { purpose and scope } \\
\text { as a foundational } \\
\text { activity }\end{array}$ & $\begin{array}{l}\text { Build consensus with } \\
\text { individuals from } \\
\text { diverse perspectives } \\
\text { about the goal and } \\
\text { scope of the curri- } \\
\text { culum }\end{array}$ & $\begin{array}{l}\text { "I think, we need to take people's } \\
\text { advice in those countries for ... } \\
\text { their circumstances and then write } \\
\text { curricula... rather than saying oh well } \\
\text { you've got to learn how to do it to } \\
\text { this standard." P001 }\end{array}$ \\
\hline $\begin{array}{l}\text { Insufficient represen- } \\
\text { tation of diverse per- } \\
\text { spectives and realities } \\
\text { in the creation of the } \\
\text { final curricula }\end{array}$ & $\begin{array}{l}\text { Fosters representati- } \\
\text { on in the curriculum } \\
\text { development stage }\end{array}$ & $\begin{array}{l}\text { Ensure diverse } \\
\text { perspectives are } \\
\text { represented in the } \\
\text { curriculum constructi- } \\
\text { on phase }\end{array}$ & $\begin{array}{l}\text { "are they just gandasizing them- } \\
\text { selves... what is their right to call it } \\
\text { global?" and "I think when you look at } \\
\text { it in a global perspective very often } \\
\text { the global is say European, North } \\
\text { American." P001 }\end{array}$ \\
\hline $\begin{array}{l}\text { A rigid conceptuali- } \\
\text { zation of competency } \\
\text { requirements }\end{array}$ & $\begin{array}{l}\text { Creates opportuni- } \\
\text { ties for mitigating } \\
\text { neo-colonial effects } \\
\text { and power differen- } \\
\text { tials }\end{array}$ & $\begin{array}{l}\text { Value local/indigen- } \\
\text { ous knowledge and } \\
\text { expertise }\end{array}$ & $\begin{array}{l}\text { "Because that's where you're } \\
\text { going to get the buy in because } \\
\text { you - everyone has saw that } \\
\text { you're appreciating everyone's sort } \\
\text { of culture and identity and I think } \\
\text { that's - because that's where the } \\
\text { difference is apart from sort of the } \\
\text { differences we discussed, which is } \\
\text { very much where you draw your } \\
\text { line of competency, that's actually } \\
\text { making sure that whenever you're } \\
\text { designing the curriculum is relevant } \\
\text { to each local team for them to have } \\
\text { buy in. But if you've got some general } \\
\text { principles that everyone agrees with } \\
\text { and general competencies, everyone } \\
\text { agrees with them, then you have the } \\
\text { local nuances about." P008 }\end{array}$ \\
\hline \multirow[t]{2}{*}{$\begin{array}{l}\text { A mismatch between } \\
\text { the curricular requi- } \\
\text { rements on local } \\
\text { context }\end{array}$} & $\begin{array}{l}\text { Create opportuni- } \\
\text { ties for addressing } \\
\text { inequities in low } \\
\text { resource countries }\end{array}$ & $\begin{array}{l}\text { Advocate for systems } \\
\text { level changes } \\
\text { including resources } \\
\text { for infrastructure to } \\
\text { underpin education }\end{array}$ & $\begin{array}{l}\text { "you can use it [the curricular docu- } \\
\text { ment] to push [stakeholders] if you } \\
\text { want to push." P014 }\end{array}$ \\
\hline & & $\begin{array}{l}\text { Ensure all countries } \\
\text { have agency and } \\
\text { self-determination }\end{array}$ & $\begin{array}{l}\text { "state of the science global docu- } \\
\text { ment and recommendations that } \\
\text { different training programs in diffe- } \\
\text { rent locations would accept, reject or } \\
\text { adapt the recommendations accor- } \\
\text { ding to their own purposes." P005 }\end{array}$ \\
\hline $\begin{array}{l}\text { The influence of power } \\
\text { relationships and } \\
\text { decision makers }\end{array}$ & $\begin{array}{l}\text { Improves the quality } \\
\text { of the implementa- } \\
\text { tion }\end{array}$ & $\begin{array}{l}\text { Factor local context } \\
\text { throughout develop- } \\
\text { ment and implemen- } \\
\text { tation phases }\end{array}$ & $\begin{array}{l}\text { "having [existing groups] share their } \\
\text { practices and reviewing the current } \\
\text { curriculum and adding value to it. I } \\
\text { think that's what's really lacking and } \\
\text { what will be really important in order } \\
\text { to make progress and again not rein- } \\
\text { venting the wheel." P011 }\end{array}$ \\
\hline
\end{tabular}




\section{Discussion}

Our data have demonstrated the complexity of global oncology curricula in both their construction and implementation. We have articulated five key challenges and outlined how diversity can be leveraged as a positive influence to mitigate these challenges (Table 1). Achieving the balance between global and local as well as contextual diversity will remain challenging however, this can be framed positively as an opportunity to interregional collaboration. If progress is made we may be able to prepare graduates to serve as advocates for their local healthcare system ${ }^{23}$.

Our data have demonstrated a critical function of global curricula is to serve as advocacy tools. Our data has demonstrated an inverse relationship between availability of health human resources and length of training. As the pressures increase to expand the cancer workforce to meet the demands for care the cancer training programs may face equal pressures to reduce their lengths of training ${ }^{1}$. This phenomenon is not unique to cancer however, investments in training in the face of health human resource shortages may ultimately counteract forces causing these shortages and result in strengthening of the workforce ${ }^{24}$. In addition to the tension between health human resource shortages and length of training, differences in local practices for length of training compared to recommendations from global curricula are a barrier to implementation. While some jurisdictions leverage these differences as an advocacy tool to protect time in training others may feel their training situations are not represented in the recommendations of the curriculum. In many areas high level political commitment to medical education is lacking ${ }^{23}$. In order to address the growing health human resource crisis in cancer, where there is a need to double the workforce ${ }^{1}$, there will need to be logistical coordination between governments, universities and training institutions as well as those who determine curriculum and assessment ${ }^{23}$. The use of global oncology curricula as a mechanism for advocacy in political and other decision making arenas as well as a vehicle for collaboration should be explicitly explored in future curricula efforts.

Neocolonialism in medical education is conceptualized as practices of exporting Western concepts and pedagogical methods without consideration of power relations ${ }^{25}$. The concept of neocolonialism is an important consideration in the creation and implementation of global curricula as identified by participants in this study. There is implicit tension in such concepts as global standards, including the generation and setting of global core competencies with educational ideals oriented to respecting local diversity and local historical educational practices ${ }^{26-27}$. The concept of a'universally global physician' who has a minimum set of essential competencies implies everyone from around the world can see themselves as represented. Operationalizing such competencies raises concern over who is represented in such recommendations and how this concept is related to broader economies of medical education (i.e. who garners material and 
symbolic benefit) ${ }^{28}$. One questions, who garners material and symbolic benefit from the packaging of competencies as universal? Our data has demonstrated that physical representation in the curriculum development process does not necessarily ensure that all relevant perspectives are reflected in the final document. While critical education scholars would support that physician competencies must reflect local socioeconomic and political contexts and local health needs ${ }^{25}$ how this is realized with the rising popularity of articulating core or universal skills is unclear. One is led to question the degree to which developing shared concepts in medical education is possible without the undue influence of Western values ${ }^{25}$. However, all participants may achieve a greater understanding if greater value is placed on embracing contextual difference in cancer education and fostering bidirectional exchanges ${ }^{29}$.

There are limitations to this work. We were not able to directly observe the process of global curriculum construction therefore could not analyze that type of activity directly. Further projects directly observing such a process would provide rich data and be of interest in advancing this field of research. In addition, we were not able to actually go to diverse geographic locations and to directly observe what occurs locally with respect to the use and implementation of global curricula. Triangulation with these other forms of data collection and analysis is an important next step for this area of research.

\section{Conclusions}

Global oncology curricula may serve important advocacy roles within the healthcare system. Leveraging diversity may positively impact the common challenges in the construction and implementation of global oncology curricula. 


\section{References}

1. Atun R, Jaffray DA, Barton MB, et al. Sept 2015. Expanding global access to radiotherapy. The Lancet. Oncology. 16(10):1153-1186.

2. Frenk J, Chen L, Bhutta ZA, et al. 2010. Health professionals for a new century: transforming education to strengthen health systems in an interdependent world. The Lancet. 376(9756):1923-1958.

3. MacCarrick GR. Dec 2010. A practical guide to using the World Federation for Medical Education (WFME) standards. WFME 1: mission and objectives. Irish journal of medical science.179(4):483-487.

4. Pavlidis N, Alba E, Berardi R, et al. 2016. The ESMO/ASCO Global Curriculum and the evolution of medical oncology training in Europe. ESMO open.1(1):e000004.

5. Giuliani M, Frambach J, Broadhurst M, Papadakos J, Fazelad R, Driessen E, Martimianakis M. A Critical Review of Representation in the Development of Global Oncology Curricula and the Influence of Neocolonialism. BMC Med Educ. 2020 Mar 30;20(1):93.

6. Giuliani M, Martimianakis M, Broadhurst M, Papadakos J, Fazelad R, Driessen E, Frambach J. Motivation and Challenges in the Development of Global Medical Curricula: A scoping review. Accepted to Academic Medicine December 2019.

7. Spring, J., 2014. Globalization of education: An introduction. Routledge.

8. Tackett S. July 2019. Examining the Educational Commission for Foreign Medical Graduates Announcement Requiring Medical School Accreditation Beginning in 2023. Academic medicine: journal of the Association of American Medical Colleges. 94(7):943-949.

9. Hodges BD, Maniate JM, Martimianakis MA, Alsuwaidan M, Segouin C. Oct 2009. Cracks and crevices: globalization discourse and medical education. Medical teacher.31(10):910-917.

10. Hartzband P, Groopman J. Aug 6 2009. Keeping the patient in the equation-humanism and health care reform. The New England journal of medicine.361(6):554-555.

11. Ho MJ, Abbas J, Ahn D, Lai CW, Nara N, Shaw K. Dec 2017. The "Glocalization" of Medical School Accreditation: Case Studies From Taiwan, South Korea, and Japan. Academic medicine : journal of the Association of American Medical Colleges. 92(12):1715-1722.

12. Prideaux D. Jan 2019. The global-local tension in medical education: turning 'think global, act local' on its head? Medical education 53(1):25-31.

13. Sefton AJ. Sept-Oct 2004. New approaches to medical education: an international perspective. Med Princ Pract. 13(5):239-248.

14. Goodman LA. 1961. Snowball Sampling. Annals of Mathematical Statistics.32(1):148.

15. Kirsti Malterud VDS, and Ann Dorrit Guassora. 2016. Sample Size in Qualitative Interview Studies: Guided by Information Power. Qualitative Health Research. 26(13):1753-1760.

16. Watling CJ, Lingard L. 2012. Grounded theory in medical education research: AMEE Guide No. 70. Medical teacher. 34(10):850-861.

17. Giuliani M, Martimianakis MA, Broadhurst M, Papadakos J, Fazelad R, Driessen E, Frambach J. Humanism in global oncology curricula: an emerging priority. Curr Oncol. 2020 
18. Richardson L and St Pierre EA. Writing:A Method Of Inquiry. In: Denizen N, Lincoln Y, ed. The Sage Handbook of Qualitative Research. 2005. Thousands Oaks: Sage Publications :959-978.

19. NVivo qualitative data analysis software; QSR International Pty Ltd. Version 11, 2015.

20. Benstead K, Turhal NS, O'Higgins N, et al. Sept 2017. Multidisciplinary training of cancer specialists in Europe. European journal of cancer. 83:1-8.

21. Eriksen JG, Beavis AW, Coffey MA, et al. Apr 2012. The updated ESTRO core curricula 2011 for clinicians, medical physicists and RTTs in radiotherapy/radiation oncology. Radiotherapy and oncology: journal of the European Society for Therapeutic Radiology and Oncology.103(1):103-108.

22. Dittrich C, Kosty M, Jezdic S, et al. 2016. ESMO / ASCO Recommendations for a Global Curriculum in Medical Oncology Edition 2016. ESMO open.1(5):e000097.

23. Celletti F, Reynolds TA, Wright A, Stoertz A, Dayrit M. Oct 2011. Educating a new generation of doctors to improve the health of populations in low- and middle-income countries. PLOS medicine.8(10):e1001108.

24. Frambach JM, Manuel BA, Fumo AM, Van Der Vleuten CP, Driessen EW. Jan 2015. Students' and junior doctors' preparedness for the reality of practice in sub-Saharan Africa. Medical teacher.37(1):64-73.

25. Karle H, Christensen L, Gordon D, Nystrup J. Oct 2008. Neo-colonialism versus sound globalization policy in medical education. Medical education.42(10):956-958.

26. Bleakley A, Brice J, Bligh J. Mar 2008. Thinking the post-colonial in medical education. Medical education.;42(3):266-270.

27. Whitehead CR. Mar 2016. On gunboats and grand pianos: medical education exports and the long shadow of colonialism. Advances in health sciences education : theory and practice.21(1):1-4.

28. Martimianakis MA, Hafferty FW. Jun 2013. The world as the new local clinic: a critical analysis of three discourses of global medical competency. Social science \& medicine. 87:31-38.

29. Naidu T, Kumagai AK. Mar 2016. Troubling Muddy Waters: Problematizing Reflective Practice in Global Medical Education. Academic medicine : journal of the Association of American Medical Colleges.91(3):317-321. 
Exploring Glocalization in the Construction and Implementation of Global Curricula ～$\quad \mathbf{1 0 3}$ 


\section{CHAPTER 6}

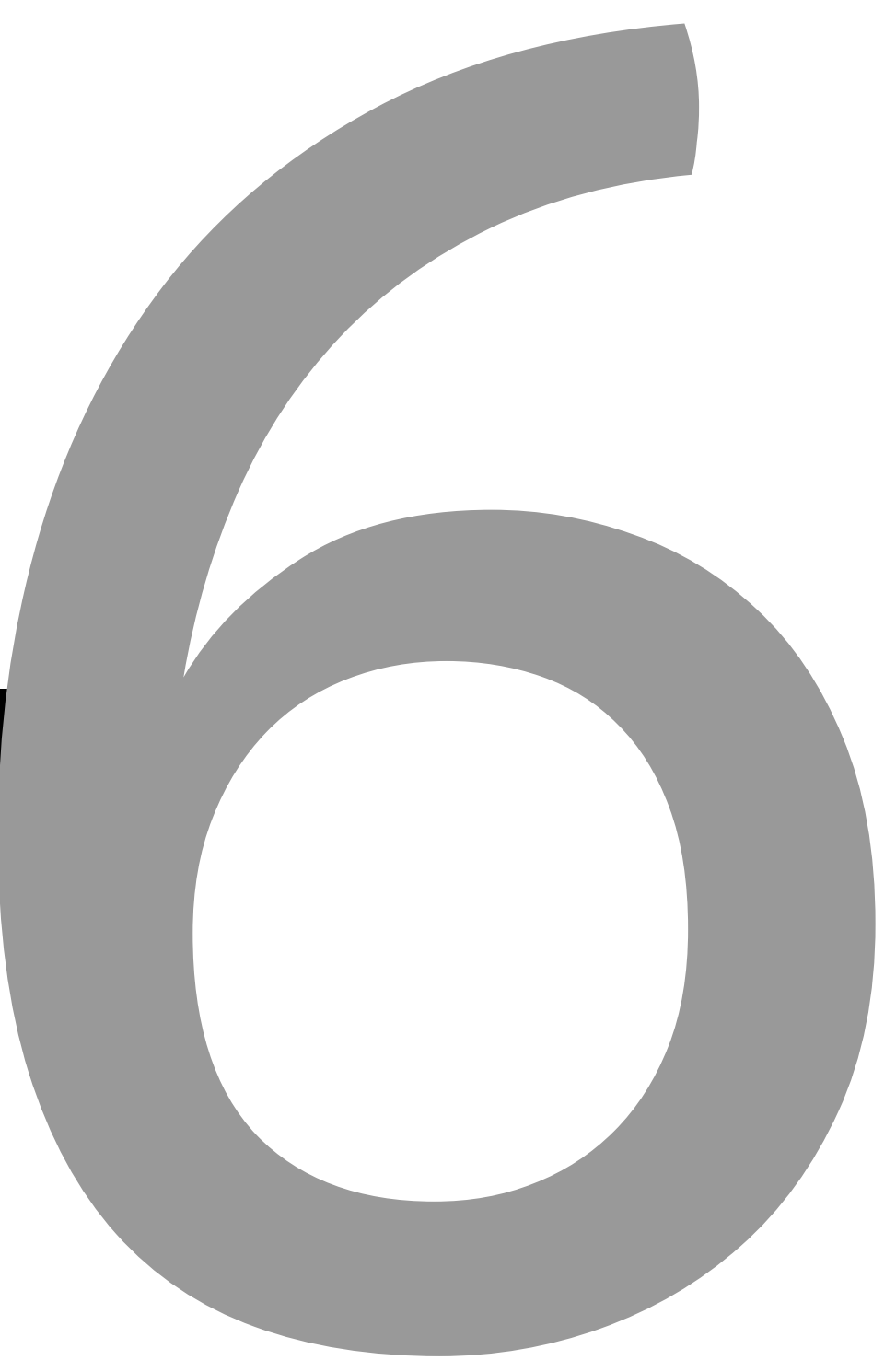




\section{Exploring Implementation of the ESTRO Core Curriculum at the National Level}

Published as: Giuliani M, Athina Martimianakis M, Benstead K, Grau Eriksen

J, Verfaillie C, Van Egten V, Umakanthan B, Driessen E, Frambach J. Exploring Implementation of the ESTRO Core Curriculum at the National Level. 


\section{Background and purpose}

Global curricula exist across medical specialties however, the factors which influence their implementation are not well understood. The purpose of this study is to report the perceived factors that impact the implementation of the ESTRO Core Curriculum.

\section{Methods}

An anonymous, 37-item, survey was designed and distributed to the Presidents of the National Societies who have endorsed the ESTRO Core Curriculum $(n=29)$. The survey addressed perceptions about implementation factors related to context, process and curriculum change. The data was summarized using descriptive statistics.

\section{Results}

Twenty-six (90\%) National Societies completed the survey. One respondent perceived that the values of the training system of their country would be incompatible with the proposed ESTRO Core Curriculum. The most common contextual barriers to implementation was a lack of support from the government (57\%), a lack of internal organizational support (35\%) and a'poor fit' between the ESTRO Core Curriculum and the broader political \& economic context (35\%). Perceived implementation process barriers included insufficient numbers of faculty (44\%), poor coordination between the government and training institutions (48\%), and a lack of an influential person leading the implementation (44\%). Two barriers related to curriculum change were a lack of funding and lack of assessment tools.

\section{Conclusions}

The content and values espoused in the ESTRO Core Curriculum are endorsed across diverse geopolitical and sociocultural regions. Barriers to curricular implementation are identified at the organizational and systems level and include insufficient teaching faculty, lack of coordination and the need for influential leadership. 


\section{Introduction}

Global or core curricula, conceptualized as a text, which intends to use a common vocabulary and shared philosophy, and which describes an outcome, including competency items, that are intended to be applicable across nations, exist in many medical specialties and represent a significant investment of time and resources to create $\mathrm{e}^{1,2}$. The motivation to create these curricula is often reported as an opportunity to define standards for a specialty, harmonize training or improve the quality of training across countries ${ }^{3}$. However, we do not understand well the real-world implications of such curricula, including factors influencing implementation and unintended effects they may create ${ }^{4}$. In addition, their applicability in diverse geopolitical and sociocultural settings is unknown ${ }^{5}$. It is possible that these curricula, which often represent consensus statements obtained through social group processes ${ }^{3}$, may reflect the priorities and values of certain dominant groups which may either limit uptake or, in some settings, may require significant adaptation for which there may not be adequate resources. The influence of such curricula in reproducing dominant perspectives on curricular content and pedagogical practices is a concern ${ }^{6,7}$. In addition, misalignment with local healthcare systems, representing diverse geopolitical and sociocultural views, are potential aspects limiting the utility of global curricula. Intercountry variation and socio-cultural differences are a potential area requiring particular attention in the implementation of global curricula as they can be challenging aspects $^{3}$. These curricula may play important advocacy roles, such as ensuring resources for training or teaching faculty, within the healthcare system ${ }^{8}$. Thus, it is important to consider how to mitigate their limitations related to implementation and uptake in diverse geopolitical contexts, in order for all countries to benefit from their advocacy potential

The health workforce gaps in oncology are projected to grow over the next decade $\mathrm{e}^{9,10}$. To address these gaps there are efforts to increase the supply of oncology healthcare providers ${ }^{11}$. One such effort is through the development of common curricula which may increase supply by facilitating physician movement or assisting in the creation of new training programs. However, the effectiveness of these curricula in resolving workforce concerns is unclear ${ }^{4,11}$. Previous work in Europe has reported that there is poor awareness of the existence of these curricula and potentially limited uptake ${ }^{12}$. In 2019, the European Society for Radiotherapy \& Oncology (ESTRO) published the fourth edition of the European Core Curriculum for Radiation Oncology/Radiotherapy (the ESTRO Core Curriculum) ${ }^{13}$. The aim of the ESTRO Core Curriculum are to "develop comparable standards for training across Europe and to facilitate free movement of specialists across borders. It is also hoped that it will improve the level of training across Europe and will make the non-"medical expert" roles more explicit" ${ }^{13}$. The ESTRO Core Curriculum has also been adopted by the European Union of Medical Specialists as the European Training Requirement for Radiation Oncology/Radiotherapy ${ }^{14}$. The publication of this new version of the curriculum presented an opportune time to explore factors which promote or 
hinder the implementation of this curricula across different countries. Understanding the factors that impact implementation may inform future efforts in creating these curricula to improve the fit for purpose. This information may also provide guidance to enhance the impact of existing curricula which represent a significant investment of resources to create. The purpose of this study is to report the perceived factors that impact the implementation of the European Core Curriculum for Radiation Oncology/Radiotherapy (the ESTRO Core Curriculum) from the perspective of the national oncology societies.

\section{Materials and Methods}

\section{Questionnaire development}

This study involved a cross-sectional online survey completed at a single time-point. Following a comprehensive literature review to identify existing empirical work and surveys addressing curriculum implementation at the global or international level, the survey was adapted from a curriculum implementation survey on the World Health Organization's (WHO) patient safety curriculum ${ }^{15}$. The adaptation included changing the survey language to focus on radiotherapy and specifically the ESTRO Core Curriculum and built on our previous work in this area ${ }^{3,16}$ and the existing literature on global curriculum implementation to address barriers commonly identified in the implementation science literature. The survey captured details, at the national level, of the current state of radiation or clinical oncology education and explored factors which may impact implementation of the ESTRO Core Curriculum including the curriculum itself, the context and the implementation process. Prior to distribution the survey was circulated to experts in oncology education for peer-review for clarity, underwent cognitive pretesting and was iteratively revised ${ }^{17}$. For the purposes of this study, Europe was defined as the members or associated members of the European Union of Medical Specialists (UEMS) from the European continent ${ }^{16}$.

\section{Survey Content}

The final survey included 37 items. The survey was conducted in English. Questions instructed respondents to "select one best response" or "select all that apply" and openended questions were also used. Some questions incorporated a 5-point Likert scale with a response scale of "strongly disagree" to "strongly agree" with an option for no response. Survey questions could be omitted or skipped by the respondents if they chose not to answer a particular question. The full survey is available in Supplementary File 1.

\section{Survey Distribution}

A research ethics board waiver (UHN 19-0388) was obtained for this study. The survey was distributed electronically to the Presidents of the National Societies for each country that endorsed European Core Curriculum for Radiation Oncology/Radiotherapy in $2011^{18}$ 
and/or in $2019^{13}$. The National Societies are the organizations that lead on the regulation of the practice of and education in radiation oncology in individual countries. The survey was therefore sent to 29 individuals from 29 countries. These countries included: Albania, Austria, Belgium, Bulgaria, Croatia, Cyprus, Czech Republic, Denmark, Estonia, Finland, France, Germany, Greece, Hungary, Ireland, Israel, Italy, Norway, Poland, Portugal, Romania, Slovakia, Slovenia, Spain, Sweden, Switzerland, The Netherlands, Turkey and The United Kingdom. The Survey was distributed in October 2019 with follow-up reminder emails and was closed in December 2019. The names and countries of the National Societies invited to participate in this survey are listed in Supplementary File 2.

\section{Analysis}

Survey data was summarized using descriptive statistics. Missing data due to non-response to an item was low and missing data due to nonresponse is reported. Countries were classified into Lower-Middle Income Countries (LMIC), Upper-Middle Income Countries (UMIC) and High-Income Countries (HIC) based on the World Bank Criteria ${ }^{19}$. All data is reported as an aggregate to ensure the anonymity of respondents.

\section{Results}

Twenty-six of the 29 country representatives completed the survey for a response rate of $90 \%$.

In 18 responses (69\%) the national speciality was radiation oncology and in $8(31 \%)$ it was clinical oncology. The duration of specialist training was 4 years $(n=6 ; 23 \%), 5$ years $(n=18$; $69 \%)$ and 6 years $(n=2 ; 8 \%)$. Twenty $(73 \%)$ countries reported having a national curriculum. Training was regulated on a national basis in 20 (77\%) countries. Of the 26 countries who had a respondent 4 (16\%) are UMIC and 22 (84\%) are HIC. No country is LMIC.

We probed barriers to curriculum implementation on three dimensions: context, process and curriculum content. 23 responses were received for this section. One (4\%) respondent reported that "Belief by leadership that the European Core Curriculum for Radiation Oncology/Radiotherapy will NOT improve oncology care" was a barrier and 5 (22\%) report that "Belief by leadership that the European Core Curriculum for Radiation Oncology/ Radiotherapy is NOT implementable" was a barrier. One respondent perceived that the values of the training system of their country would be incompatible with the proposed ESTRO Core Curriculum.

The most commonly reported contextual barriers thought to hinder implementation of the ESTRO Core Curriculum included a perceived lack of support from the government $(n=13$; $57 \%)$, a lack of internal organizational support $(n=8 ; 35 \%)$ and a 'poor fit' between the ESTRO Core Curriculum and the broader political and economic context $(n=8 ; 35 \%)$. With 
respect to the implementation process barriers 10 (44\%) reported insufficient numbers of faculty, 11 (48\%) poor coordination between the government and training institutions, and $10(44 \%)$ reported a lack of an influential person leading the implementation. All implementation factors related to context and process are summarized in Table 1.

Table 1: Implementation Factors Related to Context and Process

\begin{tabular}{ll}
\hline Context & Yes (n=23;\%) \\
\hline Lack of support from stakeholders internal to the organization. & $8(35 \%)$ \\
\hline Lack of support from stakeholders external to the organization. & $6(26 \%)$ \\
\hline Poor fit between the ESTRO Core Curriculum and the broader political \& economic context & $8(35 \%)$ \\
\hline Lack of governmental commitment to the ESTRO Core Curriculum & $13(57 \%)$ \\
\hline Lack of organization-level commitment to implementation of the ESTRO Core Curriculum. & $4(17 \%)$ \\
\hline $\begin{array}{l}\text { Belief that the ESTRO Core Curriculum is NOT compatible with the values of the training } \\
\text { system. }\end{array}$ & $1(4 \%)$ \\
\hline $\begin{array}{l}\text { Belief that the ESTRO Core Curriculum is NOT compatible with the length of training of our } \\
\text { system }\end{array}$ & $2(9 \%)$ \\
\hline $\begin{array}{l}\text { Poor fit between the ESTRO Core Curriculum and the assessment system in training } \\
\text { settings }\end{array}$ & $1(4 \%)$ \\
\hline Implementation Process & $10(44 \%)$ \\
\hline Insufficient faculty members to teach the ESTRO Core Curriculum. & $4(17 \%)$ \\
\hline Faculty are not trained on the topics to teach the ESTRO Core Curriculum. & $11(48 \%)$ \\
\hline $\begin{array}{l}\text { Poor coordination between the government and institutions around implementation of } \\
\text { the ESTRO Core Curriculum. }\end{array}$ & $7(30 \%)$ \\
\hline $\begin{array}{l}\text { Poor communication channels among stakeholders regarding implementation of the } \\
\text { ESTRO Core Curriculum. }\end{array}$ & $10(44 \%)$ \\
\hline Lack of an influential person leading implementation of the ESTRO Core Curriculum. & 20 \\
\hline
\end{tabular}

Representatives from thirteen countries (50\%) reported that they had adapted some aspects of the ESTRO Core Curriculum and that they were comfortable in doing these adaptations. The most common elements reported to require adaptation were the basic science aspects (radiobiology) or the 'high-end' radiotherapy techniques. Others reported a greater focus in their country on the Medical Expert aspects of the ESTRO Core Curriculum with less focus on the intrinsic roles such as Communicator, Leader or Professional. With respect to factors which influence curriculum change a lack of funding and assessment tools were perceived to be common barriers across countries. Figure 1 describes the implementation factors related to curriculum change.

\section{Discussion}

This study is the first to report the perceived factors that influence the implementation of the ESTRO Core Curriculum from the National Oncology Societies. We have shown that the values and curricular content presented in ESTRO Core Curriculum are perceived to be largely compatible with the healthcare education and practice settings of the participating countries despite the diverse nature of the health care systems of these 


\section{Implementation Factors Related to Curriculum Change}

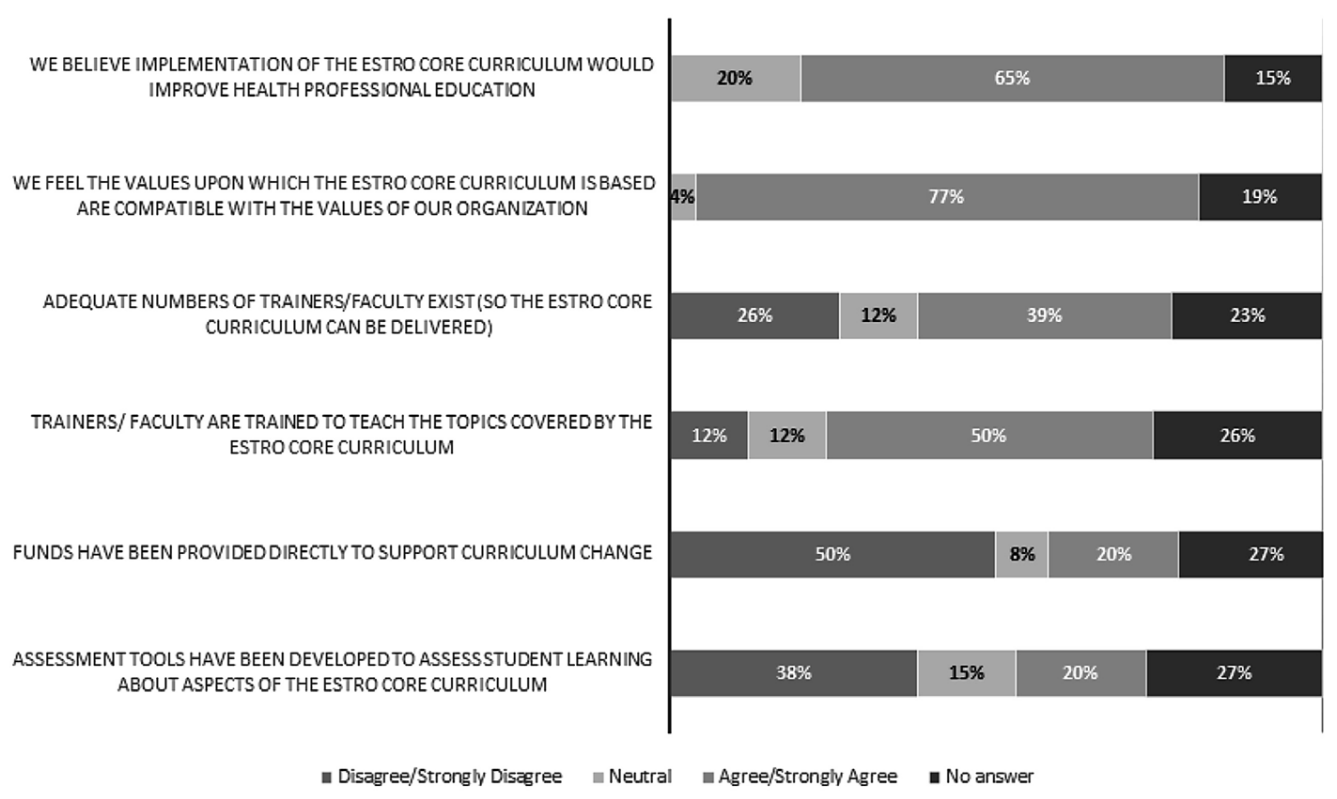

Figure 1: Implementation Factors related to Curriculum Change

countries. However, there remain several important barriers to implementation. These barriers are most commonly include insufficient teaching faculty, funding, coordination challenges between governments and training institutions and the lack of an influential leader for implementation.

Common challenges with the implementation of global or core curricula include "a mismatch between the curricular requirements and the local context" and "insufficient representation of diverse perspectives and realities in the creation of the final curricula" The data presented in this study demonstrates the curriculum development process utilized for the European Core Curriculum for Radiation Oncology/Radiotherapy has been largely successful in mitigating these challenges. First, with respect to the mismatch of requirements the majority of respondents in our study support the values, length of training recommendations and assessment system espoused in the ESTRO Core Curriculum. This is a laudable achievement as the participants in this study represent disparate geopolitical regions with vastly different sociocultural practices and health care systems with differing resources according to the $\mathrm{WHO}^{20}$. Secondly, with respect to insufficient diversity of perspectives, with each iteration of the European Core Curriculum for Radiation Oncology/Radiotherapy the number of authors engaged in developing the curricula has grown as has the diversity of representation of countries across Europe ${ }^{13,18}$. In addition to expansion of country representation other key stakeholder groups, including trainees, have been included in the development process ${ }^{13,18}$. These strategies are aligned with recommendations to enhance diversity in the development of global curricula with 
the aim to mitigate common challenges ${ }^{8}$. However, while the ESTRO Core Curriculum process appears to have addressed diversity in representation and have aligned values on the existing content more study is required to determine where the limits of the agreement on the values of the curriculum are. For example if the majority of the content focuses on clinical expertise, with the values attributed to these aspects being aligned, does this alignment hold true in areas of content that are often underrepresented in global curricula such as communication skills or competencies aligned with humanism ${ }^{21}$. When asked about adaptation locally we have data supporting a focus on the Medical Expert aspects of the curriculum versus the non-Medical Expert roles. It is not clear what the reason for this is but this would be a rich area for future study. The ESTRO Core Curriculum group is perhaps well positioned to explore more deeply other facets of oncology training including communication, approaches to end of life care and concepts such as the role of Artificial Intelligence on a multi-national level.

Our data has demonstrated that in the context of the ESTRO Core Curriculum the Presidents of the National Societies that took part in the study accept the applicability of the values and content of the curriculum. However, challenges in implementation have been identified in the areas of funding, assessment tools, a lack of teaching faculty and leadership locally. We have previously reported that there has been a growth of efforts across oncology specialties in the last two decades to create or revise global curricula. However, building on the data from this study, future efforts in the area of global curriculum in oncology may benefit from focusing on these barriers to implementation. Outside of securing additional funding for curriculum change other opportunities for efficiency, such as promoting educational global public goods, in the form of freely available educational tools and resources, may be strategies to lessen the financial burden of curricula change. Global public goods are available world-wide ${ }^{22}$. Future work may articulate areas in radiation oncology training which are amenable to becoming global public goods. Our data identified the need for assessment tools and this is perhaps one avenue to explore for sharing resources. Another example in the radiation oncology context is the challenge of delivering the required radiobiology content at local institutions. Our data has also endorsed this as a challenge. National or international courses for radiobiology exist in several jurisdictions including through the ESTRO School ${ }^{23}$ or within Canada at the University of Toronto ${ }^{24}$. These courses are a first step in addressing this challenge however barriers to access remain including cost and time to attend. Future efforts to increase access to radiobiology education may explore online, asynchronous options which may increase access to those without the funding to travel or who are working a context with limited leave for such training. The inadequate supply of teaching faculty has been previously identified as a challenge for the implementation of global curricula ${ }^{8,25}$ and is reinforced in this study. Global curricula serve important roles as advocacy tools for education at the national level ${ }^{8}$. In areas where clinical demands for care are high this 
may occur at the expense of the availability of teaching faculty. With the projected rise in cancer cases over the next decade ${ }^{9}$ this risk to educational resources is critical and is not limited to low-middle income countries ${ }^{26}$. It is possible this could create a negative cycle which further threatens the workforce where there is inadequate clinicians to meet the demands for clinical care. It may also lead to reduced availability of teaching faculty and ultimately less graduates which diminishes the inflow of resources to the workforce ${ }^{25}$. Finally, perhaps there is a role for skill building in the areas of implementation sciences and change management for those tasked with curricular change on a national level.

This work has several limitations. First, we did not ask the respondents to examine each recommendation or competency item within the ESTRO Core Curriculum to determine its feasibility nor fit within their local environment. We are able to report that overall the respondents perceive the values of the curriculum, as well as the recommendations for length of training and assessment, to be a good fit with their national system. A significant number of countries do report that they have adapted the ESTRO Core Curriculum to fit their national environments. Future studies should explore in detail the nature and process of these adaptations as this data may be critical to inform future iterations of the curricula and highlight areas where more time should be allocated for discussion in the development phase. Due to the methodology of this study we were not able to address one of the major challenges in global curriculum development and implementation which is the influence of power relationships and implicit biases in the development process ${ }^{8}$. This is a critical area of study but is better suited to alternative methodologies such as critical ethnography. Future efforts to revise and update the ESTRO Core Curriculum may be an opportune time for such a study. Finally, this survey was sent to the Presidents of the National Societies for each country that endorsed the ESTRO Core Curriculum. We acknowledge their responses are one, albeit an influential, perspective in the discussion around curriculum implementation. We do not purport to have identified all perspectives from the multiple groups involved in curriculum implementation on a national level. In addition, we have not attempted to access data from countries who may be using the ESTRO Core Curriculum, beyond those who have endorsed the ESTRO Core Curriculum, and believe this would be an important focus of future studies. A particular focus may be on LMIC countries whose perceptions are not reflected in the data presented in this study.

The content and values espoused in the European Core Curriculum for Radiation Oncology/Radiotherapy are endorsed across diverse geopolitical and sociocultural regions by the National Societies. Barriers to curricular implementation are identified at the organizational and systems level and include insufficient teaching faculty, poor coordination between governments and training institutions and the need for influential leadership. 


\section{References}

1. "World Directory of Medical Schools-WFME Projects." The World Federation for Medical Education, wfme.org/world-directory/. [Accessed 10 January 2019]

2. "The Institute for International Medical Education." World Health Organization, World Health Organization, 23 July 2011,www.who.int/workforcealliance/members_partners/member_list/ iime/en/. [Accessed 8 January 2019]

3. Giuliani M, Martimianakis M, Broadhurst M, Papadakos J, Fazelad R, Driessen E, Frambach J. Motivation and Challenges in the Development of Global Medical Curricula: A scoping review. Accepted to Academic Medicine December 2019.

4. Patel, Shilpen, et al. "The International Core Literature Consensus (ICLC): an alternative curriculum for oncologists." Journal of Cancer Education 26.3 (2011): 420. https://www.doi. org/10.1007/s13187-011-0242-y

5. Giuliani M, Frambach J, Broadhurst M, Papadakos J, Fazelad R, Driessen E, Martimianakis M. A Critical Review of Representation in the Development of Global Oncology Curricula and the Influence of Neocolonialism. BMC Med Educ. 2020 Mar 30;20(1):93.

6. Hartzband, Pamela, and Jerome Groopman. “Keeping the patient in the equation-humanism and health care reform." New England Journal of Medicine 361.6 (2009): 554. https://www.doi. org/10.1056/NEJMp0904813

7. Whitehead, Cynthia. "Scientist or science-stuffed? Discourses of science in North American medical education." Medical education 47.1 (2013): 26-32. https://www.doi.org/10.1111/j.13652923.2011.04136.x

8. Giuliani M., Frambach J., Driessen E., Martimianakis M. "Exploring Glocalization in the Construction and Implementation of Global Curricula". J Cancer Educ. 2020 Feb 11. doi: 10.1007/ s13187-020-01705-5.

9. Atun, Rifat, et al. "Expanding global access to radiotherapy." The Lancet Oncology 16.10 (2015): 1153-1186. https://www.doi.org/10.1016/S1470-2045(15)00222-3

10. Wilson, Brooke E., et al. "Estimates of global chemotherapy demands and corresponding physician workforce requirements for 2018 and 2040: a population-based study." The Lancet Oncology 20.6 (2019): 769-780. https://www.doi.org/10.1016/S1470-2045(19)30163-9

11. Garson Jr, Arthur. "Perspective: leveraging the health care workforce: what do we need and what educational system will get us there?." Academic Medicine 86.11 (2011): 1448-1453. https://www.doi.org/10.1097/ACM.0b013e318230588b

12. Bibault JE., Franco P., et al. "Learning radiation oncology in Europe: Results of the ESTRO multidisciplinary survey." Clinical and translational radiation oncology 9 (2018): 61-67. https://www. doi.org/10.1016/j.ctro.2018.02.001

13. Benstead, Kim, et al. "Recommended ESTRO Core Curriculum for Radiation Oncology/ Radiotherapy 4th edition." Radiotherapy and Oncology 141 (2019): 1-4. https://www.doi. org/10.1016/j.radonc.2019.08.013 
14. "Training Requirements for the Specialty of Radiation Oncology." European Union of Medical Specialists, 2013, https://www.uems.eu/_data/assets/pdf_file/0015/44430/UEMS-2013.20-European-Training-Requirements-in-Radiation-Oncology.pdf [Accessed 14 Jan 2020]

15. Ginsburg, Liane R., Neelam Dhingra-Kumar, and Liam J. Donaldson. “What stage are low-income and middle-income countries (LMICs) at with patient safety curriculum implementation and what are the barriers to implementation? A two-stage cross-sectional study." BMJ open 7.6 (2017): e016110. http://dx.doi.org/10.1136/bmjopen-2017-016110

16. Benstead, Kim, et al. "Multidisciplinary training of cancer specialists in Europe." European Journal of Cancer 83 (2017): 1-8. https://www.doi.org/10.1016/j.ejca.2017.05.043

17. Fowler F. Survey Research Methods. Thousand Oaks, CA: SAGE Publications; 2013.

18. Eriksen, Jesper G., et al. "The updated ESTRO core curricula 2011 for clinicians, medical physicists and RTTs in radiotherapy/radiation oncology." Radiotherapy and Oncology 103.1 (2012): 103-108. https://www.doi.org/10.1016/j.radonc.2012.02.007

19. "World Bank Country and Lending Groups." World Bank Country and Lending Groups - World Bank Data Help Desk, datahelpdesk.worldbank.org/knowledgebase/articles/906519-worldbank-country-and-lending-groups. [Accessed 13 December 2019]

20. World Health Organization. The world health report 2000: health systems: improving performance. World Health Organization, 2000. [Accessed 5 December 2019]

21. Giuliani M, Martimianakis MA, Broadhurst M, Papadakos J, Fazelad R, Driessen E, Frambach J. Humanism in global oncology curricula: an emerging priority. Curr Oncol. 2020

22. Kaul I, Grunberg I, Stern M. "Global public goods: international cooperation in the 21st century". NY Oxford University Press, Inc. ; 1999.

23. "Basic Clinical Radiobiology 2020." ESTRO-European Society for Radiotherapy \& Oncology. www. estro.org/Courses/Basic-Clinical-Radiobiology-(1). [Accessed 18 December 2019]

24. "Clinical and Experimental Radiobiology Course 2020." Clinical and Experimental Radiobiology Course | Radiation Oncology, www.radonc.utoronto.ca/clinical-and-experimental-radiobiology-course. [Accessed 18 December 2019]

25. OECD. Publishing. The Looming Crisis in the Health Workforce: How Can OECD Countries Respond?. Organisation for Economic Co-operation and Development, 2008. https://www.who. int/hrh/migration/looming_crisis_health_workforce.pdf [Accessed 9 December 2019]

26. Fundytus, Adam, et al. “Delivery of global cancer care: an international study of medical oncology workload." Journal of global oncology 4 (2017): 1-11. https://www.doi.org/10.1200/ JGO.17.00126 


\section{Supplementary File 1: Implementing the European Core Curriculum for Radiation Oncology/ Radiotherapy (the ESTRO Core Curriculum) Survey}

We are seeking to understand the factors that impact the implementation the European Core Curriculum for Radiation Oncology/Radiotherapy (the ESTRO Core Curriculum). In particular, this short questionnaire contains questions about various aspects of implementing:

1. The European Core Curriculum for Radiation Oncology/Radiotherapy Third Edition (2011) or Fourth Edition (2019).

2. Other oncology curricula that countries/universities have developed

Your responses will assist in understanding factors that influence curriculum implementation.

There are 37 questions in this survey.

\section{Section 1: Respondent Characteristics}

1. What country are you completing this survey for?

2. What is your professional role?

3. Is the radiotherapy specialty in your country? (check all that apply)

- Radiation Oncology

- Clinical Oncology

4. Approximately, how many new trainees are trained in your country annually or radiation/clinical oncology?

5. How many training institutions are there in your country in 2019 for radiation/clinical oncology? 
6. What is the duration of specialist training (after MD/MBBS exam or completion of medical school) in your country for radiation/clinical oncology? (choose one of the following answers)
- 3 years
$\circ 4$ years
05 years
06 years
○ Other:
- No answer

7. Do you have a national curriculum?
○ Yes
○ No
- No answer

8. Is competence to complete training assessed by (check all that apply):

\begin{tabular}{llll}
\hline \multicolumn{1}{c}{ Yes } & Uncertain & No & No answer \\
\hline Oral examination & & & \\
\hline Written examination & & \\
\hline Work place based assessments & & \\
\hline Other & & \\
\hline
\end{tabular}

9. Is training regulated on a (check all that apply):
- National basis
- Regional basis
- Institutional basis

10. What authority confirms completion of training/final qualifications in your country?

11. Does the specialist certification from your country allow you to work in other European countries?
- Yes
- No
- No answer 


\section{Section 2: Barriers to Implementation}

This section relates specifically to barriers to implementing the ESTRO Core Curriculum. A barrier is an obstacle that prevents or gets in the way of achieving something. Please indicate which items are/were barriers to adopting or implementing the ESTRO Core Curriculum for your training systems (check all that apply)

a) Potential barriers elated to the curriculum itself? (check all that apply)

- 1. Belief by leadership that the ESTRO Core Curriculum is NOT implementable.

- 2. Belief by leadership that the ESTRO Core Curriculum will NOT improve oncology.

Describe any other important barriers to implementation related to the ESTRO_Core Curriculum itself:

b) Potential barriers related to the Context (check all that apply).

- 3. Lack of support from stakeholders internal to the organization.

- 4. Lack of support from stakeholders external to the organization.

- 5. Poor fit between the ESTRO Core Curriculum and the broader political \& economic context (such as regulation of health professionals, faculty remuneration, etc.).

- 6. Lack of governmental commitment to the ESTRO Core Curriculum (for example - providing ongoing financial support).

- 7. Lack of organization-level commitment to implementation of the ESTRO Core Curriculum.

- 8. Belief that the ESTRO Core Curriculum is NOT compatible with the values of the training system.

- 9. Belief that the ESTRO Core Curriculum is NOT compatible with the length of training of our system.

- 10. Poor fit between the ESTRO Core Curriculum and the assessment system in training settings (for example - the University).

Describe any other important barriers to implementation of the Curriculum related to characteristics of the organization or external environment: 
c) Potential barriers related to the Implementation Process (check all that apply).

- 11. Insufficient faculty members to teach the ESTRO Core Curriculum.

- 12. Faculty are not trained on the topics to teach the ESTRO Core Curriculum.

- 13. Poor coordination between the government and institutions around implementation of the ESTRO Core Curriculum.

- 14. Poor communication channels among stakeholders regarding implementation of the ESTRO Core Curriculum.

- 15. Lack of an influential person leading implementation of the ESTRO Core Curriculum.

Describe any other important barriers to implementation related to the process of implementing the ESTRO Core Curriculum and how they were a barrier:

\section{Section 3: Curricular Implementation Stage}

1. If your country is implementing a Radiation/Clinical Oncology Curriculum that is different from the ESTRO Core Curriculum, please briefly describe the curriculum (e.g. source, total number of years, content, etc.

2. Have you adapted any aspect of the ESTRO Core Curriculum? If yes, what parts?

3. What was the motivation for adapting the ESTRO Core Curriculum?

4. Are there components of the ESTRO Core Curriculum you think are not compatible you're your local context? If yes, which parts and please describe why there are not compatible?

5. Did/do you feel comfortable with adapting the ESTRO Core Curriculum to your local context? Why/why not?

6. What is your interpretation of the concept of "implementation of the ESTRO Core Curriculum" in your national context? 


\section{Section 4: Implementation Factors}

1. Considering Curricular Change

Please indicate the extent to you "Agree" or "Disagree" with each of the following:

\begin{tabular}{|c|c|c|c|c|c|c|}
\hline & $\begin{array}{l}\text { Strongly } \\
\text { disagree }\end{array}$ & Disagree & Neutral & Agree & $\begin{array}{l}\text { Strongly } \\
\text { Agree }\end{array}$ & No answer \\
\hline $\begin{array}{l}\text { 1. We believe implementation } \\
\text { of the ESTRO Core Curriculum } \\
\text { would improve health profes- } \\
\text { sional education. }\end{array}$ & & & & & & \\
\hline $\begin{array}{l}\text { 2. We feel the values upon } \\
\text { which the ESTRO Core Curri- } \\
\text { culum is based are compa- } \\
\text { tible with the values of our } \\
\text { organization. }\end{array}$ & & & & & & \\
\hline
\end{tabular}

1. Describe any other activities that were undertaken to help decide whether your country would implement the ESTRO Core Curriculum.

2. Is there anything else that you think is relevant to the implementation of the ESTRO Core Curriculum in your country?

3. Is there anything the central ESTRO Office could do to facilitate use or implementation of the ESTRO Core Curriculum in your country? 


\section{Supplementary File 2: National Societies Endorsing the European Core Curriculum for Radiation Oncology/Radiotherapy}

\begin{tabular}{|c|c|c|}
\hline Country & Society & Website \\
\hline Albania & Albanian Oncology Association & www.aoa.org.al \\
\hline Austria & Austrian Society for Radiation Oncology (ÖGRO) & http://www.oegro.com/ \\
\hline Belgium & Belgian Society of Radiation Oncology (BeSTRO) & https://bestro.be \\
\hline Bulgaria & The Guild of Bulgarian Radiotherapists (GLB) & \\
\hline Croatia & Croatian Society of Oncology (HOD) & $\begin{array}{l}\text { http://hrvatsko-onkolosko-drustvo.com/ } \\
\text { hr/index.php }\end{array}$ \\
\hline Cyprus & Cyprus Oncology Society & \\
\hline Czech Republic & $\begin{array}{l}\text { The Czech Society for Radiation Oncology, Bio- } \\
\text { logy and Physics (SROBF) }\end{array}$ & http://www.srobf.cz/ \\
\hline Denmark & Danish Society of Clinical Oncology (DSKO) & www.dsko.org \\
\hline Estonia & Estonian Society of Oncology & \\
\hline Finland & $\begin{array}{l}\text { Finnish Society for Radiotherapy and Oncology } \\
\text { (SOY) }\end{array}$ & \\
\hline France & $\begin{array}{l}\text { French Society for Radiotherapy and Oncology } \\
\text { (SFRO) }\end{array}$ & http://www.sfro.org/ \\
\hline Germany & $\begin{array}{l}\text { German Society for Radiotherapy and Oncology } \\
\text { (DEGRO) }\end{array}$ & www.degro.org \\
\hline Greece & Hellenic Society for Radiation Oncology (EEAO) & http://www.eeao.gr \\
\hline Hungary & $\begin{array}{l}\text { Hungarian Society for Radiation Oncology } \\
\text { (HUSRO) }\end{array}$ & http://www.oncol.hu/husro/index2.htm \\
\hline Ireland & $\begin{array}{l}\text { The Radiation Oncology Committee of the Facul- } \\
\text { ty of Radiologists, Royal College of Surgeons in } \\
\text { Ireland (acting as National Society) }\end{array}$ & http://www.radiology.ie/ \\
\hline Israel & $\begin{array}{l}\text { Israeli Society of Clinical Oncology and Radiothe- } \\
\text { rapy (ISCORT) }\end{array}$ & www.iscort.org.il \\
\hline Italy & $\begin{array}{l}\text { Italian Association of Oncological Radiotherapy } \\
\text { (AIRO) }\end{array}$ & http://www.radioterapiaitalia.it/ \\
\hline Norway & Norwegian Society for Oncology (NOF) & $\begin{array}{l}\text { http://legeforeningen.no/Fagmed/ } \\
\text { Norsk-onkologisk-forening/ }\end{array}$ \\
\hline Poland & $\begin{array}{l}\text { General Council of Polish Society of Radiation } \\
\text { Oncology (PSTRO) }\end{array}$ & www.ptro.io.gliwice.pl \\
\hline Portugal & $\begin{array}{l}\text { Portuguese Society of Radiotherapy Oncology } \\
\text { (SPRO }\end{array}$ & www.spro.pt \\
\hline Romania & $\begin{array}{l}\text { RSRMO-Romanian Society for Radiotherapy and } \\
\text { Medical Oncology }\end{array}$ & http://www.srrom.ro/ \\
\hline Slovakia & Slovak Society of Radiation Oncology & http://www.radiacnaonkologia.sk/ \\
\hline Slovenia & SSRO-Slovene Society for Radiation Oncology & \\
\hline Spain & $\begin{array}{l}\text { Spanish Association of Radiotherapy and Onco- } \\
\text { logy (SEOR) }\end{array}$ & www.seor.es \\
\hline Sweden & Swedish Society of Oncology (SOF) & http://onkologi.org/ \\
\hline Switzerland & $\begin{array}{l}\text { Scientific Association of Swiss Radiation Onco- } \\
\text { logy (SASRO) }\end{array}$ & http://www.sgsmp.ch/ \\
\hline The Netherlands & $\begin{array}{l}\text { Dutch Society for Radiotherapy and Oncology } \\
\text { (NVRO) }\end{array}$ & http://www.nvro.nl/ \\
\hline Turkey & Turkish Society for Radiation Oncology (TROD) & www.radonk.org \\
\hline UK & The Royal College of Radiologists (RCR) & http://www.rcr.ac.uk/ \\
\hline
\end{tabular}


CHAPTER 7

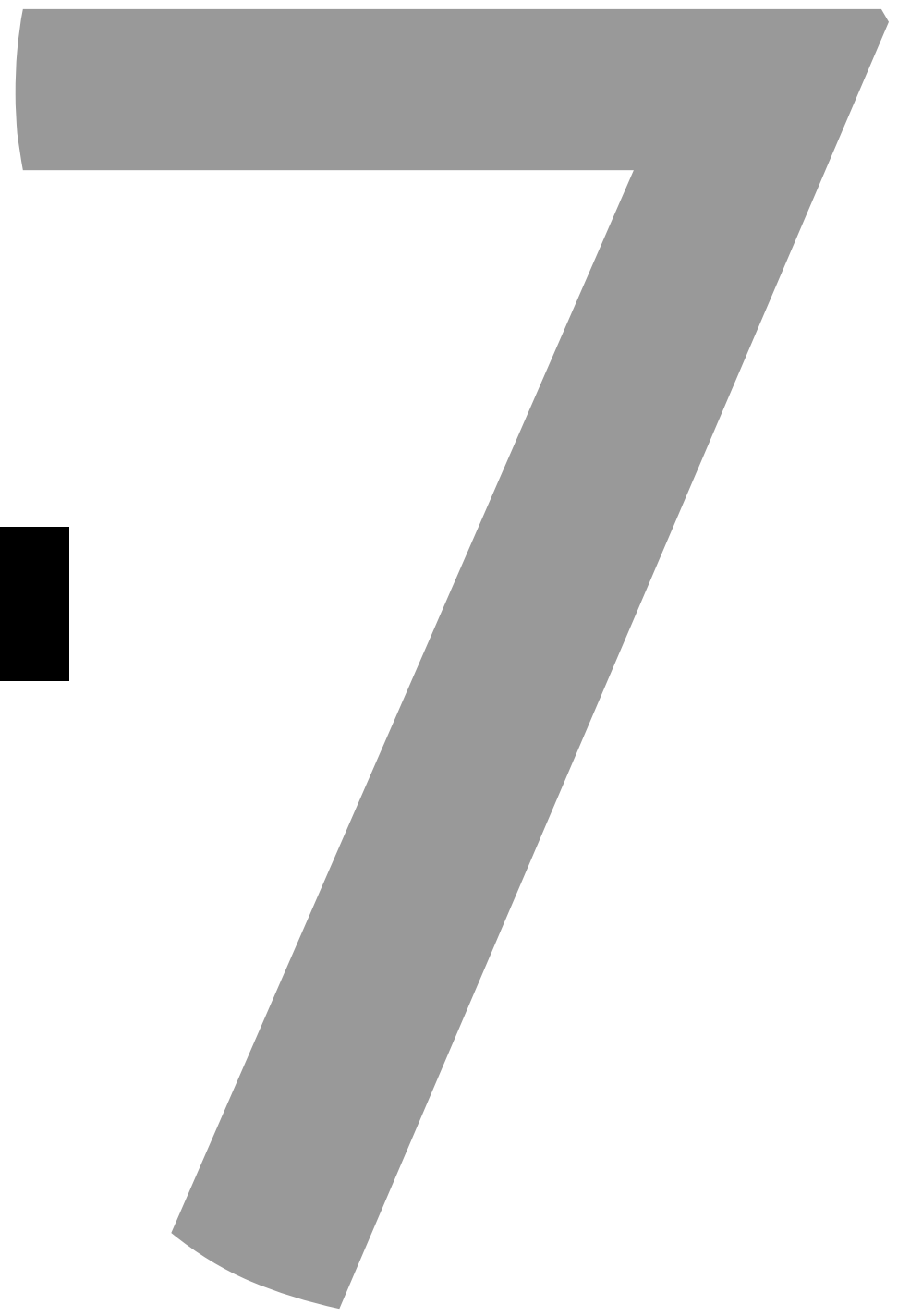


Discussion 
The overall aim of this dissertation was to explore the motivations behind and the unintended effects of global curricula in oncology. Using a critical approach, inspired by the work of anti-colonial scholars, I have investigated how power and bias influence the development and implementation of global oncology curricula. Through a series of five studies, I have addressed my research objectives. This chapter summarizes the main findings with respect to the research questions, discusses conclusions which can be drawn from this work and addresses the strengths and limitations of this dissertation. I conclude with a presentation of possible avenues for future research.

\section{Main Findings}

\section{Research Question 1: What are the motivations and perceived premises for a global medical curriculum?}

Inspired by an anti-colonial approach we sought to understand what the authors who published on the topic of global medical education curricula articulate as the purpose for these activities and the challenges. This was an important starting place for our work because it provides insight, across medical disciplines and regions, about what priorities and focus was dominant in the literature. Through our scoping review we were able to systematically map and report the trends in global medical curricula. The findings, presented in chapter 2 , identify that the purposes most frequently reported for creating global medical curricula were to define specialty specific standards, to harmonize training standards, or to improve the quality of training. The most common challenges were intercountry variation including differences in the healthcare system, theoperationalization of medical training and socio-cultural differences, the need for a multi-stakeholder approach and implementation. In these publications we see a predominance of North American and European perspectives. Our data source was the published literature. Our approach, a scoping review, in itself is open to critique as what is published and therefore legitimated is not representative of the diverse global perspectives, particularly voices of low resource countries. However, it does allow us to engage with the dominant voice and the existing mechanism for legitimating and disseminating knowledge or positions. We expand on these concepts and limitations later in this chapter. Our conclusions from this chapter led us to question, given the growing global incidence of cancer and the impending crisis in access to care, what global curricula exist for oncology and who has developed them.

\section{Research Question 2: What global curricula exist for oncology? Who has developed them and what methods were used in their development?}

Our findings presented in chapter 2 supported our hypothesis that curricular homogenization is one factor driving the movement to create global curricula. We reported the influence of Western authors and organizations on existing efforts across 
medical specialties. To address research question 2 we focused on three specialities of oncology, namely medical, radiation and surgical oncology and sought to identify full curricula in the public domain. Our findings, presented in chapter 3, addressed the issue of representation in global oncology curricula. We reported that the majority of authors contributing to global oncology curricula are male and from European or North American countries. We confirmed the findings from chapter 2 that the most common curricular purposes were promoting quality patient care, harmonization of training standards, and facilitating physician mobility. We also reported that the methods for creation of these curricula were most commonly a committee or task force. Using a comparative analysis over time we documented an increase in the proportion of female authors and the number of countries represented in the authorship. We concluded that existing global oncology curricula are heavily influenced by Western authors and as a result may not incorporate relevant socio-cultural perspectives impacting care in diverse geographic settings.

We inferred that origin of authorship is a tangible proxy for power asymmetries ${ }^{1}$ in the creation of these curricula. However, we wished to explore in greater depth the content of these global oncology curricula to understand and compare what competencies are and are not included and what inferences can we draw from this with regard to local applicability and the representation of diverse local perspectives in global curricula.

\section{Research Question 3: What competencies are and are not included in the existing global curricula for oncology?}

The dominance of the biomedical model of medical education, which has its origin in North America and Europe, is an important aspect in the discussion of globalization and medical education. We reported in chapters 2 and 3 that existing global curricula are created predominantly through social group processes and that authors from North American or European countries make up the majority of the authors. We were therefore interested in the curricular content within the global oncology curricula. We hypothesized that the biomedical aspects of cancer care would be predominant, at the expense of other qualities and competencies such as humanistic qualities. In this chapter we reported that of 7792 competencies items across 17 oncology curricula $10 \%$ aligned with humanistic competencies. The humanistic items were primarily focused on the attributes of Respect, Compassion and Empathy. We also identified a conflation between the humanistic competencies and the professionalism domain from the CanMEDS framework. These findings are suggestive of the biomedical paradigm, and physician as scientist, occupying a large proportion of the curricula. While we cannot comment on what an ideal distribution of competencies would be within a global curriculum, the existing distribution can be criticized for paying minimal attention to roles outside the medical expert. These findings may inform the discussion around re-evaluating curricular priorities to better fit the 
needs of patients and the healthcare system proposed in the Lancet Commission Health Professionals for a new Century².

The findings from this study, which built upon studies one and two, contributed to our appreciation of the degree of representation in the creation of global curricula, their proposed purpose and now the predominant content. From these studies we wished to explore in greater detail the way in which global curricula were conceptualized and the factors which impact their adoption in diverse geopolitical arenas.

\section{Research Question 4: How are global oncology curricula conceptualized and implemented and their relationship to local contexts of power and culture?}

In conducting the studies presented in chapters 2 to 4 it became clear that conceptualization and implementation of global oncology curricula was not well described in the published literature. We believe that neocolonial effects may be hidden from us because the published literature, which was the data source for studies one to three, is heavily dominated by North American and European perspectives steeped in the biomedical discourse $\mathrm{s}^{3,4}$. The published literature limited our ability to access and attend to non-dominant or marginalized perspectives and voices ${ }^{1}$.

As such, we employed an interview-based approach to problematize the concept and implementation of global oncology curricula and their relationship to local contexts of power and culture. The findings, presented in chapter 5 , articulated 5 key challenges for global curricula including 1) ambiguous or conflicting perspectives on the purpose and scope of global oncology curricula 2) insufficient representation of diverse perspectives and realities in the creation of the final curricula 3) a rigid conceptualization of competency requirements 4) a mismatch between the curricular requirements and local context and 5) the influence of power relationships and decision makers. These challenges speak to the issues we identified in the previous chapters including a lack of diversity in contributor or author representation in the creation of global curricula and a predominance of content focused on the biomedical paradigm.

In addition to these challenges, we articulated how diversity can be leveraged to foster representation, addressing power differentials and factoring local contexts as an approach to mitigating these challenges. These practical recommendations may be applied to future curriculum development efforts.

Following this work, we wished to explore in greater depth, from the perspective of those who had been charged at a national level with implementing global oncology curricula, their perception of the factors which influenced implementation. 


\section{Research Question 5: What are the factors that influence global oncology curriculum implementation?}

In chapters 2 to 5 a recurring, emerging issue was the challenges of implementation of global oncology curricula. However, other than identifying implementation as a challenge, data was not available about more specific aspects of these implementation challenges. In chapter 6 we conduct an international, cross-sectional survey study. The purpose of this study was to report the perceived factors that impact the implementation of the European Core Curriculum for Radiation Oncology/Radiotherapy (the ESTRO Core Curriculum) from the perspective of the National Oncology Societies. We identified that the content and values espoused in the ESTRO Curriculum are endorsed across diverse geopolitical regions. However, barriers to curricular implementation are identified at the organizational and systems level and include insufficient teaching faculty, lack of coordination and the need for influential leadership. These data demonstrate that in an organization that has progressively increased the geopolitical diversity of representation in their efforts to revise their core curriculum they have achieved support for the content and values which they cover in the curriculum. Applying an anti-colonial lens to these conclusions, we concluded that attention must continue to be paid to the process by which consensus is achieved and the potential for loss of local diversity which may be critical for healthcare delivery. Power relationships must still be attended to in the development and implementation of global curricula. We were able to identify further areas for research which may focus on implementation science and change management training for those tasked with implementing the ESTRO Core curriculum at the national level. We also again identified the role global curricula can play as advocacy tools, in particular, for teaching faculty resources and funding for curriculum changes.

\section{Conclusions}

A number of conclusions and implications can be drawn from the work presented in this dissertation. The overall aim was to explore the motivations behind the generation of global oncology curricula and the unintended effects of these documents. The five studies conducted in this dissertation have identified several patterns. First, what is considered legitimate knowledge and is therefore prioritized is heavily oriented to the Euro-American, Western perspective. There is a focus on medical expertise underpinned by the biomedical model which may be a factor in the mismatch between curricula and local healthcare needs. Next, diversity plays a critical role in the development and implementation of global curricula. Without attending to diversity there is a mismatch between what is described and what is needed or even possible in local contexts. However, as demonstrated in chapter 6, where there is deliberate effort to attend to diversity of representation, some of the challenges described in chapter 5 are mitigated while other challenges remain. Finally, global curricula can have a role as an advocacy tool within the healthcare system. They can be used to secure faculty time for training, training positions 
and other resources. We have identified that resources for implementation remain a significant challenge for global curricula and using these curricula as advocacy tools on the national and international level may be a solution. The concluding thoughts from this dissertation are organized into critical and practical considerations and will end with future avenues for research.

\section{Critical considerations}

Taking a critical stance, we have identified that the concept of a global curriculum is a complex construct that has different interpretations in different contexts. It is unlikely that creating a single, universally applicable set of recommendations for pursuing global curricula, or a universally applicable curricular content, will address the gaps in cancer care and training faced around the world. However, efforts to create and update global curricula are likely to continue and attending to the themes we have identified in this dissertation may offer productive avenues for enhancing the utility of these efforts. These include consideration of whose knowledge is perceived as legitimate, the tendency for the medical expert role to dominate curricular content, the biomedical paradigm to overpower considerations of diversity in health care approaches, the role of diversity in developing local relevance for global curricula, and the leveraging of global curricula as advocacy tools.

We have identified that the authors of existing global curricula are heavily oriented to the Euro-Western location. This supports previous authors' claims that in the era of homogenization and standardization of medical education we are experiencing neocolonialism in medical education ${ }^{5}$. When discussing the colonial legacy of medical education in India, Anshu et al refer to "a clash of cultures where the East was seen as weak against the powerful knowledge of the West" ${ }^{\prime \prime}$. They go on to articulate how perceptions of inferior knowledge and the desire by medical professionals to be perceived as competent resulted, not in collaboration, synthesis and mutual understanding, but in borrowing in the absence of meaningful dialogue ${ }^{6}$. This resulted in what the authors term as a perception of "second class doctors" and a further reduction in access to care by patients ${ }^{6}$. This is but one tangible example of the challenges and potential adverse effects of efforts in medical education that are not reflexive and do not attend to diversity. Taking these challenges further, medical education practices articulated in the literature and otherwise, are often presented as internationally applicable, universally accepted and unbiased ${ }^{7}$. However, if one is reflexive and critical in their review of this, one concludes this is unlikely to be case. For example, curriculum development work, grounded in social group processes, are influenced by participant biases. Going further, we conclude the content of global oncology curricula is heavily oriented to the biomedical paradigm or more specifically the Western biomedical paradigm. With on-going calls for curricular reform ${ }^{2,8}$, and a desire to 
create education which "transcends national and political boundaries"5 the gap between curricula and health systems needs ${ }^{9}$ may be worsened if these issues are not attended to.

The content of existing global oncology curricula is oriented to the medical expert domain rooted in the biomedical paradigm. The global need for increase oncology workforce capacity will continue and with it so will efforts to create and revise global oncology curricula. To address the gaps in radiotherapy access alone over 200,000 new health care professionals are needed by $2035^{10}$. This workforce need is a supply and demand problem ${ }^{11}$. The majority of the work around this problem currently occurs on the supply side attempting to increase the number of graduates. These traditional efforts to increase the availability of health care professionals have led to only a minimal increase in health human resources, attrition of trained individuals from areas of highest need, a lack of sustainability, and often ill-equipped graduates to address the needs of patients and the public ${ }^{2}$. Efforts to address the workforce shortages in oncology may benefit from re-examining the workforce equation of supply and demand. Instead of a sole focus on increasing supply there may be avenues to reduce demand by equipping individuals and teams with different skills. This may involve task shifting, integration of health systems science, a focus on equity and a move from a model dominated by biomedical approaches to care to a model that integrates other ways of caring for the ill $^{12}$. Future efforts in global curriculum design will likely be influenced by the calls for a focus on social accountability or health systems science ${ }^{13}$. For example, Miller et al produced a framework which is focused on undergraduate medical education but provides a practice framework including concepts of "Global Consensus for Social Accountability of Medical School", and designing through participatory action research and appreciative inquiry ${ }^{14}$. These concepts ${ }^{15}$ incorporate techniques which may facilitate representations of previously repressed voices. However, they are recommendations from a North American focus and must be critically interpreted. In 2019 the United Nations issued a special report on health workforce education calling for a refocus of education centered on a rights based approach. They make a number of key recommendations including "ensure health education curricula provide a balance between public health, preventive health, community and social medicine, mental health, palliative care, medical ethics, medical law, managerial skills, communication skills, and human rights"16. However, while the framework proposed in this UN report is laudable medical educators have been struggling for decades with the concept of reform.

With the WHO's recent publication of objectives on a global strategy for human resources these curricula require review in the content of these recommendations ${ }^{17}$. We have demonstrated even with more health professionals it is unlikely we will be able to train enough to meet projected demand with our current training and care models. Innovative efforts should be focused on the demand side of the equation ${ }^{11}$. There are several proposals 
to reduce the demand side of the equation including reducing training time and task shifting. Garson has stated that training should be "long enough and no longer" and that local customization is critical to realizing this ${ }^{11}$. Emanuel et al in 2012 called for medical schools training to refocus attention on essential elements ${ }^{18}$. Our data has demonstrated that over time the amount of curricular content and competencies within global curricula has grown and that the medical expert domain remains the dominant focus ${ }^{19}$. This reflects the deep roots of the biomedical model and its dominance over other skills and types of knowledge ${ }^{20}$. If we are not able to realize a model that is flexible and adapts to local, essential needs we will not achieve closure of gaps in care ${ }^{14}$.

Leveraging diversity in the participants who create global curricula and in the way in which these curricula are conceptualized may address some of the challenges for implementation we describe above. This may require changes to the ways groups have previously been engaged in these activities. We have reported that social group processes such as Delphi techniques are frequently used to create global curricula. However, these processes are vulnerable to bias and power dynamics. Pan et al have described the use of the Nominal Group Technique, which is a form of social group process, but which by design may be better able to attend to diverse views ${ }^{21}$. We acknowledge that it is effortful to employ new techniques and methods for curriculum development. However, creation of global curricula represents large investment of time and resources and efforts to maximize their utility are warranted.

The concepts of the relationship between knowledge producers and their audience is not unique to curriculum design. This complexity has been nicely articulated with respect to research in global health and can be applied to curriculum design. Abimbola, when discussing global health research, articulates 3 manifestations of colonial attitudes ${ }^{1}$. These are: 1) "foreign experts who write about local issues without acknowledging how and why their real audience is actually not local experts, but other foreign experts, 2) foreign experts who set out to write for an audience of local experts without acknowledging the limitations of what they can possibly know about local realities and 3) local experts who write for a foreign audience, without acknowledging how that imagined audience influences what they study and what they write" ${ }^{\prime 1}$. We believe our work support that these manifestations may apply to global curriculum design. The first and second manifestation we articulate in chapters 2 and 3 where Western authors are producing global curricula through a process which cannot fully attend to local priorities and knowledge. Manifestation 3 is an important facet which has not been explored in the work in this dissertation nor in the literature that we have reviewed. In addition, interpersonal power dynamics and their perception at the individual level are complex. As an outsider observing, we may perceive an individual to be repressed but this may not be their interpretation or feeling about the 
situation. We do not presume to have identified all the ways diverse perspectives may be silenced or all of the ways in which an individual may experience these interactions.

Finally, we describe global curricula as tools which may serve an advocacy role in assisting local contexts in securing additional resources including teaching faculty, salary for trainees, protected time for trainee education and other resources to deliver education. This is important as these resource limitations remain a barrier to curriculum implementation; even when the curriculum has been developed embracing the concept of diversity as we describe in chapter 6 . However, caution is needed here as well. Anshu et al, discussing the Indian context, describe how such laudable advocacy efforts may result in unintended effects. They describe a significant challenge of faculty shortages for medical teaching. To overcome faculty shortages and to meet regulatory requirements for teaching faculty this results in behaviors such as imposter faculty or faculty poaching using exorbitant salary incentives ${ }^{6}$.

\section{Practical considerations}

This dissertation has presented some tangible, practical considerations for future efforts in the development and implementation of global curricula which may be a step in addressing the Western dominance of previous efforts. These include considering representation across a wide range of geopolitical and sociocultural views to ensure diversity of perspectives in the generation of curricula. We go further to postulate that merely inviting individuals with diverse views will not be sufficient to address the gaps in diversity. The process of curricular creation will need measured and considered facilitation to ensure that dominant voices do not negate other viewpoints. We believe global curricula will continue to be created and revised as the oncology profession works to address workforce shortages. It is important to consider these practice opportunities to advance and improve future efforts in this area.

We report common challenges in the development and implementation of global curricula including 1) ambiguous or conflicting perspectives on the purpose and scope of global oncology curricula 2) insufficient representation of diverse perspectives and realities in the creation of the final curricula 3) a rigid conceptualization of competency requirements 4) a mismatch between the curricular requirements and local context and 5) the influence of power relationships and decision makers. In addition, there are challenges with mismatch of expected requirements and the financial resources available to support the training and practice of these requirements.

Leveraging diversity, in all its forms including geopolitical, sociocultural and gender are a practical approach to mitigating some of the challenges associated with global curricula. We will address an approach to each of the challenges. First, ambiguous or 
conflicting perspectives on the purpose and scope of global oncology curricula requires the foundational activity of the group to clarify the curricular purpose and scope. Second, insufficient representation of diverse perspectives and realities in the creation of the final curricula can be mitigated by conscious and deliberate efforts to ensure that people with diverse perspectives and expertise are able to participate in the curricular process. This may require efforts beyond mere invitation of different individuals as the barriers to authentic participation in such processes can be myriad including financial, language and clinical care coverage considerations. Third, a rigid conceptualization of competency requirements, for example the degree of ability to independently implant a prostate for low-dose-rate brachytherapy upon completion of training, is a challenge and implicit biases in these conceptualizations should be addressed. This approach is an opportunity to mitigate neocolonial effects of global curricula. To achieve this there must be an authentic and psychologically safe environment where participants embrace diverse ways to knowing and applying knowledge. Fourth, a mismatch between the curricular requirements on local context is a challenge which if addressed represents an opportunity to address inequalities in countries with low resources. In mitigating this challenge, global curricula can be used as an advocacy tool to work for systems level changes including resources for infrastructure to underpin education. In addition, if the curriculum serves as a'roadmap' as described by Berman there is room for adaptation and integration locally ${ }^{22}$. Finally, the influence of power relationships and decision makers on the implementation of global curricula can be mitigated by addressing diverse local contexts throughout the curricula development and implementation phases.

We have described in chapter 6 that curriculum development groups that have focused on diversity of representation have success in alignment across diverse geopolitical areas on curriculum values and content. This success should not be taken for granted that it will yield rewards for all parties involved following implementation. We recommend that the process for the curriculum development incorporate attention to power relations and have a structure to deliberations that ensures diversity in need is not lost during moments in the process where the focus is on reaching consensus. The operating principle should be that standard setting is not the same as standardization ${ }^{23}$. The organizers or sponsors should explicitly consider diversity of perspectives for participation in the development process and how to ensure appropriate diversity has been achieved. Specifically, the process for discussion, facilitation and decision-making approach should be reviewed and the final process should include deliberate attention to power differentials and preserving diversity of perspective. Finally, those organizing these efforts should consider a focus and dedicating resources to the implementation aspects such as political advocacy, sharing of resources and skills development for those tasked with ensuring curricular change. 


\section{Limitations of the work}

This work has several limitations. The first is our position as Canadian and European researchers, a privileged position with respect to voice that limits our ability to fully engage in an anti-colonial analysis. As individuals who identify as Canadian and European we acknowledge our inability to align fully with all world view perspectives on these topics, especially perspectives which may be considered indigenous or marginalized. However, an anti-colonial approach, as an anti-oppressive stance, has given us an important lens with which to analyze existing efforts to engage in global oncology curriculum development and implementation which has not been similarly studied by other authors. Our application of anti-colonial theory more closely approximates an approach to resist slipping into a dominant voice during the course of the research and not tied to advocating for or protecting specific indigenous knowledges or experiences ${ }^{24}$. We identify with the position of Hart et al. who articulates all parties in the colonial relationship can engage in anti-colonial work. Those who identify as settlers, work to educate themselves and members of their groups. Thus, they are serving a supporting role in this field ${ }^{25}$. We believe our work has added to medical education literature in this capacity. In addition, we are constrained by the dominant positivist ontology and epistemology in the field on oncology. As we developed our work, with the hopes of speaking to oncologists and oncology educators and a desire to be published in oncology journals we had to balance these differences in ontology and epistemology. The approach to scholarly work in oncology identifies with detachment and abstraction as described by Carlson ${ }^{26}$. We struggled to apply anticolonial theory while working within the constraints of both the oncology field and the publication structures and priorities of oncology journals. The desire and perceived need to be published in a specific type of journal operated in contrast to the need for deep rigour with anti-colonial approaches with which we framed this research. We acknowledge this tension ultimately influenced our work. We hope in the future, this work will serve as a foundation to advance research that foregrounds power relations and that offers a more nuanced line of inquiry within the field of oncology.

The second limitation is that we have used the term regionality, to classify areas of the world as Western or non-Western. This has clear limitations as there can be significant heterogeneity among countries within large regions like Europe. This level of classification does not speak to which areas are health resource rich or poor and what influence an analysis at this level may have on the representation, conceptualization and implementation of global curricula. We acknowledge the limitations of using outdated typologies for appreciating the complexity of contemporary geopolitical relationships. Problematizing classification traditions is a rich avenue for future research. There are further limitations with respect to our use of the categories of Western and non-Western. One's ability to claim certain pedagogical practices or priorities as purely Western is likely too simplistic and may in itself perpetuate neocolonialism. In fact, to use the biomedical 
model as an example, one may be engaging in a colonizing activity by claiming the entire biomedical model as Western. In the studies in this dissertation we have attempted to problematize concepts of representation and content by applying an anti-oppressive, anti-colonial lens to reveal how power and privilege may dominate the current landscape for global curricula. However, we were limited in our ability to ascertain which aspects of the homogenization discourse apply to diverse local context. As a result, we cannot provide guidance on how best to construct future global curricula. As postulated by Frambach et al. in regard to problem based learning (PBL) one can also suppose that a single and universal concept of 'global curricula' is unrealistic ${ }^{27}$. We are able to identify the need and potential benefit for greater diversity represented in the social processes of curricular development but recognize such processes remain vulnerable to implicit and explicit biases by participants. In addition, there are other key stakeholder voices including trainees and patients ${ }^{28}$ who are not reflected in the work conducted in this dissertation.

Thirdly, we have focused our work in the postgraduate phase of training pre-certification. The medical training spectrum from undergraduate certification through to maintenance of certification and local regulatory requirements for practice are all critical areas for studying the influence of globalization and the impact of power dynamics. We do not presume that our findings are directly applicable across the entire spectrum of medical training but suggest that these are important aspects for consideration at all levels of training and post-certification regulation of medical practice.

Finally, as previously acknowledged, we have engaged with and built some of our work from the published literature. There are imbalances in academic global authorship which stem from entrenched power asymmetries ${ }^{1}$ and as such not all perspectives will be represented in the published literature. We acknowledge the curricula and publications which formed the bases of chapters 2 to 4 represent a specific piece of global reality ${ }^{1}$ and that more work is needed to holistically explore this space from diverse perspectives. We do believe that by starting our work in the published literature we were able to make explicit imbalances and potential unintended influence of the existing paradigms of global oncology curriculum design.

\section{Future Directions}

This dissertation has identified that existing efforts to develop global oncology curricula are heavily influenced by North American and European authors and the content is rooted in the Western biomedical paradigm. Future work might explore in greater depth how power relationships are realized and mitigated, or not, in the social processes of global curricular development and implementation. This could be accomplished through a critical ethnographic approach for example. Future work may also explore what the field of oncology globally would recognize as excellence in medical education. Such a 
study could problematize assumptions about what knowledge and skills are perceived and marketed as critical for oncologists and what attributes then inform education's concept of excellence in oncology training. Through a 'contrastive education' approach, which is an approach to explore differences and heterogeneity ${ }^{5}$, scholars can facilitate the celebration of global differences and conceptualize how to realize these differences in global curricula. Conceptual domains such as humanism, which are intrinsically linked to culture and society, and their role in global curricula may be well served by such an approach.

Global oncology curricula are complex educational endeavours. The work in this dissertation has critically explored the assumptions and challenges with these curricula and has provided some practical recommendations to inform future work in this area. While the ultimate goal is to improve care for patients with cancer and to meet the needs of this growing demographic we must not forget that diversity of perspective and humanistic aspects of care are important in these efforts. 


\section{References}

1. Abimbola S. The foreign gaze: authorship in academic global health. BMJ Glob Health. 2019;4(5):e002068.

2. Frenk J, Chen L, Bhutta ZA, et al. Health professionals for a new century: transforming education to strengthen health systems in an interdependent world. Lancet. Dec 04 2010;376(9756):1923-1958.

3. Pai M. Global Health Research Needs More Than A Makeover. In: Forbes, ed: https://www. forbes.com/sites/madhukarpai/2019/11/10/global-health-research-needs-more-than-a-makeover/\#930bcb77e34d; 2019.

4. Jones J, Cunsolo A, Harper SL. Who is research serving? A systematic realist review of circumpolar environment-related Indigenous health literature. PLoS One. 2018;13(5):e0196090.

5. Alan Bleakley JBaJB. Medical Education for the Future: Identity, Power and Location. Vol New York: Springer; 2011.

6. Anshu S, A. Evolution of medical education in India: The impact of colonialism. Journal of Postgraduate Medicine. 2016;62(4):255-259.

7. Hodges BD, Maniate JM, Martimianakis MA, et al. Cracks and crevices: globalization discourse and medical education. Med Teach. Oct 2009;31(10):910-917.

8. Cooke M, Irby DM, Sullivan W, et al. American medical education 100 years after the Flexner report. N Engl J Med. Sep 28 2006;355(13):1339-1344.

9. Bleakley A, Brice J, Bligh J. Thinking the post-colonial in medical education. Med Educ. Mar 2008;42(3):266-270.

10. Atun R, Jaffray DA, Barton MB, et al. Expanding global access to radiotherapy. Lancet Oncol. Sep 2015;16(10):1153-1186.

11. Garson A, Jr. Perspective: Leveraging the health care workforce: what do we need and what educational system will get us there? Acad Med. Nov 2011;86(11):1448-1453.

12. Daneil Hollenberg LM. Epistemological challenges to integrative medicine: An anti-colonial perspective on the combination of complementary/alternative medicine with biomedicine. Health Sociology Review. 2010;19(1):34-56.

13. Education as a Catalyst for Health Care Transformation. Harvard Macey Institute; 2019.

14. Miller BM, Eichbaum Q, Brady DW, et al. Aligning health sciences education with health needs in developing countries. Acad Med. Nov 2011;86(11):e10.

15. Wen LS, Greysen SR, Keszthelyi D, et al. Social accountability in health professionals' training. Lancet. Dec 3 2011;378(9807):e12-13.

16. Nations U. Human rights-based approaches to health workforce education. file:///C:/Users/giuliam/Downloads/UNSR\%20MedicalEducation_Factsheet_FINAL.pdf. 2019.

17. WHO. Global Strategy on human resources for health: Workforce 2030. In: Organization WH, ed: Geneva, Switzerland 2016.

18. Emanuel EJ, Fuchs VR. Shortening medical training by 30\%. JAMA. Mar 21 2012;307(11):11431144. 
19. Giuliani M, Martimianakis MA, Broadhurst M, Papadakos J, Fazelad R, Driessen E, Frambach J. Humanism in global oncology curricula: an emerging priority. Curr Oncol. 2020

20. Whitehead C, Kuper A. Beyond the biomedical feedlot. Acad Med. Nov 2012;87(11):1485.

21. Hui Pan JLN, You-Sin Liang, Jia-Ning Li \& Ming-Jung Ho. Building a professionalism framework for healthcare providers in China: A nominal group technique study. Medical Teacher. 2013;35:e1531-e1536.

22. Berman A. Core Curriculum:Globalization orGlocalization...or Both? Clinical Anatomy. 2017;30:562-563.

23. Seftan Timmermans RA. Objectification, standardization, and commodification in health care: A conceptual readjustment. Social Science \& Medicine. 2009;69:21-27.

24. George Sefa Dei AA. The power of Social Theory: The Anticolonial Discursive Framework. Jounral of Educational Thought. 2001;35(3).

25. Michael A Hart SSaGR. Working Across Contexts: Practice Considerations of Doing Indigenist/ Anti-Colonila Research. Qualitative Inquiry. 2017;23(5):332-342.

26. Carlson E. Anti-Colonial methodologist and practices for settler colonial studies. Settler Colonial Studies. 2017;7(4):496-517.

27. Frambach JM, Talaat W, Wasenitz $S$, et al. The case for plural PBL: an analysis of dominant and marginalized perspectives in the globalization of problem-based learning. Adv Health Sci Educ Theory Pract. Oct 172019.

28. International Federation of Medical Students A, European Medical Students A, Hilgers J, et al. European core curriculum - the students' perspective, Bristol, UK, 10 July 2006. Med Teach. Mar 2007;29(2-3):270-275. 



\section{Summary}

Critical scholars in medical education are presenting growing arguments on the influence of neocolonialism in medical education. As we reflect on the popularized theories of curriculum development and the reforms, including the Flexner report, that have shaped our current, Western, standards for medical education training we see many unintended effects. These include the marginalization and exclusion of women and minorities from training and a growing mismatch between the needs of patients and the health system and curriculum. Oncology provides a rich environment to study neocolonialism because global curricula have been generated in this field and have been implemented with mixed success. With the impending global crisis of health human resources in cancer there is a popular focus on training and curricula at the global level. One may postulate that perhaps the imposition of Western values globally may perpetuate neocolonial relationships, which may drive a mismatch between global priorities and local needs. Using a critical, anti-colonial stance, we have explored the perceived premises for global curricula and existing challenges. By using oncology as a case example, we investigated unintended aspects of pursuing global curricula without actively considering the historical sociopolitical relationships that have perpetuated differences in health professional education around the world.

Chapter 1. In this chapter we reviewed the background for inquiry in globalization in medical education, the influence of neocolonialism and a critical discussion on curriculum design. These considerations support the core questions shaping this these which include:

1). How are global medical curricula premised by stakeholder groups and what is their purported purpose? 2) Whose voices are represented in consensus work to develop global oncology curricula? 3) How are global oncology curricula conceptualized and implemented and what are their relationship to local contexts of power and culture? 4) What are the factors that influence global oncology curriculum implementation? The five research questions of the dissertation are then presented. These include: 1) What are the motivations and perceived premises for a global medical curriculum? 2) What global curricula exist for oncology? Who has developed them and what methods were used in their development? 3) What competencies are and are not included in the existing global curricula for oncology? 4) How are global oncology curricula conceptualized and implemented and their relationship to local contexts of power and culture? And 5) What are the factors that influence global oncology curriculum implementation? We concluded 
with a reflexivity statement by the principal author and how her experiences, professional roles and 'insider status' influenced the work in this dissertation.

Chapter 2: In chapter 2 we set out to understand the alignment of purpose with process in the development of global medical curricula including considering the socio-political differences of participants. Through a scoping review methodology we explored the challenges in creating such curricula and implications for local relevance. This chapter addresses research question 1 "What are the motivations and perceived premises for a global medical curriculum?". With 137 papers we identify that curricula were developed by a social group process (such as committee) or a Delphi process. The purpose for creating global medical curricula was most commonly to define specialty specific standards, to harmonize training standards, or to improve the quality of training. The most common challenges were intercountry variation including differences in the healthcare system, the operationalization of medical training and socio-cultural differences, the need for a multi-stakeholder approach and implementation. Such considerations undoubtedly impact uptake of the curricula and can only be addressed by explicit effort to make global curricula applicable to the realities of diverse health care sectors.

Chapter 3: In this chapter we build on the considerations raised in chapter 2 and focus on oncology as a case study. We conducted a systematic review to identify global oncology curricula and using an anti-colonial lens explore the issue of representation in global oncology curricula and alignment of purpose of these curricula and development methods. This chapter contributed to our understanding of research question 2 "What global curricula exist for oncology? Who has developed them and what methods were used in their development?". 32,835 publications were identified in the search and 17 remained following application of the inclusion/exclusion criteria. Eleven of 17 papers were published from 2010-2018 and 13 curricula originated from Europe. The 17 curricula had 300 authors; 207 were male and most were from Europe ( $n=190 ; 64 \%)$ or North America ( $n=73 ; 24 \%)$. The most common curricular purposes we identified were promoting quality patient care $(n=11)$, harmonization of training standards $(n=10)$, and facilitating physician mobility $(n=3)$. The methods for creation of these curricula were most commonly a committee or task force $(n=10)$. Over time there was an increase in the proportion of female authors and the number of countries represented in the authorship. We concluded that existing global oncology curricula are heavily influenced by Western male authors and as a result may not incorporate relevant socio-cultural perspectives impacting care in diverse geographic settings.

Chapter 4: In chapter 4 we performed an in-depth, framework analysis of the 17 global oncology curricula identified in chapter 3. Through this analysis we explored the incorporation of humanistic competencies in global oncology curricula, their relationship 
to CanMEDS roles and how these priorities and associations may reproduce Western medial priorities.

chapter 4 addresses research question 3 "What competencies are and are not included in the existing global curricula for oncology?"We identify 7792 curricular items, in 17 curricula of which $780(10 \%)$ aligned with the I.E.C.A.R.E.S humanism framework. The proportion of humanistic items in individual curricula ranged from $2 \%$ to $26 \%$ and the proportion increased from $3 \%$ in the oldest to $11 \%$ in the most recent curricula. Within the CanMEDS domains, the majority of humanistic items aligned with Professional (35\%), Medical Expert (31\%) or Communicator (25\%). The proportion of humanistic competencies has been increasing in global oncology curricula over time, however the overall proportion remains low and represents a largely Western perspective on what constitutes humanism in healthcare. The representation of humanism primarily focuses on the I.E.C.A.R.E.S attributes of Respect, Compassion and Empathy.

Chapter 5: As our work on chapters 2 to 4 progressed, addressing research questions 1 to 3 , it became clear there was a need to more deeply and richly explore how global curricular are conceptualized, challenges and perceived mitigating factors to these challenges. In chapter 5 we problematize the concept and implementation of global oncology curricula and their relationship to local contexts of power and culture. This was achieved through in-depth, one-on-one semi-structured interviews. Through iterative analyses the meaning and implication of the themes were discussed and refined to yield a conceptual analysis of the neo-colonial relationships reproduced and/or perpetuated through educational efforts to develop and implement global oncology curricula. Chapter 5 addressed research question 4 "How are global oncology curricula conceptualized and implemented and their relationship to local contexts of power and culture?" Through these interviews we identified 5 key challenges for global curricula including 1) ambiguous or conflicting perspectives on the purpose and scope of global oncology curricula 2) Insufficient representation of diverse perspectives and realities in the creation of the final curricula 3) A rigid conceptualization of competency requirements 4) A mismatch between the curricular requirements and local context and 5) The influence of power relationships and decision makers. Leveraging the strengths of diversity including fostering representation, addressing power differentials and factoring local contexts may be an approach to mitigating these challenges. We concluded global oncology curricula may serve important advocacy roles within the healthcare system. Leveraging diversity may positively impact the common challenges in the construction and implementation of global oncology curricula.

Chapter 6: In chapters 2 to 5 a recurring issue that was identified was the challenge of implementation of global oncology curricula. However, other than identifying 
implementation as a challenge, data was not available about more specific aspects of these implementation challenges. In chapter 6 we conduct an international, crosssectional survey study. The purpose of this study was to report the perceived factors that impact the implementation of the European Core Curriculum for Radiation Oncology/ Radiotherapy (the ESTRO Core Curriculum) from the perspective of the National Oncology Societies. We identified that the content and values espoused in the ESTRO Core Curriculum are endorsed across diverse geopolitical regions. However, barriers to curricular implementation are identified at the organizational and systems level and include insufficient teaching faculty, lack of coordination and the need for influential leadership. These data demonstrated that in an organization that has progressively increased the geopolitical diversity of representation in their efforts to revise their core curriculum they have achieved support for the content and values which they cover in the curriculum. Applying an anti-colonial lens to these conclusions, attention must continue to be paid to the process by which consensus is achieved and the potential for loss of local diversity which may be critical for healthcare delivery. Power relationships must still be attended to in the development and implementation of global curricula. Following this success in process leadership we were able to identify further areas for research and reform, including a focus on implementation science and change management training for those tasked with curriculum implementation and change at the national level. We also again identified the role global curricula can play as advocacy tools, in particular, in the areas on teaching faculty resources and funding for education and training.

Chapter 7: In Chapter 7 we summarized and presented a discussion on how chapters 2 to 6 have addressed the research questions and will address the overall strengths and limitations of the work presented in this dissertation. A concluding review of how this work has advanced what is known in the development and implementation of global oncology curricula is provided. We end the dissertation with a discussion of next steps in this field of study. 


\section{Samenvatting}

Kritische wetenschappers op het gebied van het medisch onderwijs komen met steeds meer argumenten ten aanzien van de invloed van het neokolonialisme in het medisch onderwijs. Al nadenkend over de breed omarmde curriculumontwikkeling-enherzieningstheorieën, zoals het Flexner-rapport, die onze huidige, westerse normen voor het geven van medisch onderwijs hebben vormgegeven, zien we dat deze veel onbedoelde gevolgen hebben gehad. Denk bijvoorbeeld aan de marginalisatie en uitsluiting van vrouwen en minderheden van het onderwijs en de steeds slechtere afstemming tussen patiëntbehoeften enerzijds en het zorgsysteem en het curriculum anderzijds. Oncologie biedt een rijke omgeving voor de studie van het neokolonialisme, omdat op dit vakgebied internationale curricula ontwikkeld zijn die met wisselend succes zijn ingevoerd. Door het dreigende wereldwijde tekort aan gezondheidswerkers op het gebied van kanker is er momenteel een populaire focus op onderwijs en curricula op internationaal niveau. Verondersteld zou kunnen worden dat het wereldwijd opleggen van westerse waarden mogelijk leidt tot een bestendiging van neokoloniale betrekkingen, wat op zijn beurt weer een discrepantie tussen mondiale prioriteiten en plaatselijke behoeften in de hand kan werken. Vanuit een kritische, antikoloniale positie onderzochten we wat de waargenomen uitgangspunten voor internationale curricula waren, alsook de bestaande uitdagingen. Door oncologie als voorbeeldcasus te nemen, onderzochten we welke onbedoelde aspecten de invoering van internationale curricula met zich mee heeft gebracht, doordat daarbij niet actief werd nagedacht over de traditionele sociaal-politieke betrekkingen die verschillen in het gezondheidszorgonderwijs wereldwijd hebben bestendigd.

Hoofdstuk 1: In dit hoofdstuk bespraken we de achtergrond voor onderzoek naar globalisering van het medisch onderwijs, alsmede de invloed van het neokolonialisme, en hielden we een kritische discussie over curriculumontwerp. Deze overwegingen ondersteunen de volgende kernvragen die dit proefschrift vormgeven:

1) Wat zijn volgens groepen van belanghebbenden de uitgangspunten voor internationale geneeskundecurricula en wat zijn volgens hen de doelstellingen daarvan?; 2) Wiens inbreng is vertegenwoordigd in consensuswerk dat de ontwikkeling van internationale oncologiecurricula ten doel heeft?; 3) Hoe worden internationale oncologiecurricula geconceptualiseerd en ingevoerd en hoe verhouden deze curricula zich tot de plaatselijke machts- en cultuurcontexten?; 4) Welke factoren beïnvloeden de invoering van een internationaal oncologiecurriculum? Vervolgens worden de vijf onderzoeksvragen van het proefschrift gepresenteerd, te weten: 1) Wat zijn de drijfveren 
en waargenomen uitgangspunten voor een internationaal Geneeskundecurriculum?; 2) Welke internationale curricula bestaan er voor oncologie? Wie heeft deze ontwikkeld en welke methoden werden daarbij gebruikt?; 3) Welke competenties worden wel en niet opgenomen in bestaande internationale oncologiecurricula?; 4) Hoe worden internationale oncologiecurricula geconceptualiseerd en ingevoerd en hoe verhouden deze curricula zich tot plaatselijke machts- en cultuurcontexten?; en 5) Welke factoren beïnvloeden de invoering van een internationaal oncologiecurriculum? We sloten het hoofdstuk af met een reflexiviteitsverklaring door de hoofdauteur waarin zij inging op de vraag hoe haar ervaringen, professionele rollen en "insider-status" het werk in dit proefschrift hebben beïnvloed.

Hoofdstuk 2: In Hoofdstuk 2 trachtten we te begrijpen hoe bij de ontwikkeling van een internationaal Geneeskundecurriculum doelstellingen en processen op elkaar werden afgestemd, bijvoorbeeld door rekening te houden met de sociaal-politieke verschillen tussen deelnemers. Met behulp van een verkennende literatuurstudie (scoping review) onderzochten we de uitdagingen bij het ontwikkelen van dergelijke curricula en de gevolgen voor plaatselijke relevantie. In dit hoofdstuk werd onderzoeksvraag 1 beantwoord: "Wat zijn de drijfveren en waargenomen uitgangspunten voor een internationaal Geneeskundecurriculum?" Op basis van 137 artikelen concludeerden we dat curricula werden ontwikkeld door middel van een sociaal groepsproces (bijvoorbeeld door een commissie) of door een Delphi-proces. De reden voor het ontwikkelen van internationale Geneeskundecurricula was meestal om specialisme-specifieke normen vast te stellen, opleidingsnormen te harmoniseren of om de kwaliteit van de opleiding te verbeteren. De meest voorkomende uitdagingen waren de verschillen tussen landen, zoals verschillen in het zorgstelsel, de operationalisering van de medische opleiding alsmede sociaal-culturele verschillen, de behoefte om meerdere belanghebbenden in het proces te betrekken en de invoering. Dergelijke overwegingen hebben ongetwijfeld gevolgen voor de invoering van deze curricula en er zal alleen iets mee worden gedaan als men zich nadrukkelijk inspant om internationale curricula toepasbaar te maken op de realiteit van de diverse sectoren in de gezondheidszorg.

Hoofdstuk 3: In dit hoofdstuk bouwen we voort op de overwegingen die in Hoofdstuk 2 naar voren zijn gebracht en richten we ons op oncologie als casestudy. We verrichtten een overzichtsstudie om internationale oncologiecurricula in kaart te brengen en om door een antikoloniale lens de kwestie van vertegenwoordiging in internationale oncologiecurricula en de afstemming van de doelstellingen en ontwikkelmethodes van deze curricula te onderzoeken. Dit hoofdstuk hielp ons om onderzoeksvraag 2 beter te begrijpen: "Welke internationale curricula bestaan er voor oncologie? Wie heeft deze ontwikkeld en welke methoden werden daarbij gebruikt?" Bij onze zoektocht onderscheidden we 32.935 publicaties, waarvan er na toepassing van de inclusie/exclusiecriteria 17 overbleven. Elf 
van deze 17 artikelen waren tussen 2010 en 2018 gepubliceerd en 13 curricula waren afkomstig uit Europa. De 17 curricula hadden 300 auteurs; 207 daarvan waren man en de meesten kwamen uit Europa ( $n=190 ; 64 \%)$ of Noord-Amerika ( $n=73 ; 24 \%)$. De voornaamste curriculumdoelstellingen die we onderscheidden waren het bevorderen van kwalitatief hoogwaardige zorg $(n=11)$, het harmoniseren van opleidingsnormen $(n=11)$ en het bevorderen van de mobiliteit van artsen $(n=3)$. De methoden waarmee deze curricula werden ontwikkeld bestonden hoofdzakelijk uit een commissie of taakgroep $(n=10)$. In de loop der tijd nam het aantal vrouwelijke auteurs en het aantal landen waaruit de auteurs afkomstig waren toe. Onze conclusie was dat bestaande internationale oncologiecurricula sterk beïnvloed worden door westerse, mannelijke auteurs en dat deze curricula dientengevolge mogelijk niet de relevante sociaal-culturele perspectieven weerspiegelen die de zorg in verschillende geografische settingen beïnvloeden.

Hoofdstuk 4: In Hoofdstuk 4 verrichtten we een verdiepende kaderanalyse van de 17 in Hoofdstuk 3 onderscheiden internationale oncologiecurricula. Met behulp van deze analyse onderzochten we in hoeverre humanistische competenties waren opgenomen in deze curricula, hoe deze competenties zich verhielden tot de CanMEDS-rollen en hoe geconstateerde prioriteiten en verbanden zouden kunnen zorgen voor een reproductie van westerse medische prioriteiten. In Hoofdstuk 4 werd onderzoeksvraag 3 beantwoord: "Welke competenties worden wel en niet opgenomen in bestaande internationale oncologiecurricula?" Binnen de 17 curricula onderscheidden we 7.792 curriculumonderdelen waarvan er 780 (10\%) overeenstemden met het humanistische kader I.E.C.A.R.E.S. Het aantal humanistische onderdelen in de afzonderlijke curricula varieerde van $2 \%$ tot $26 \%$ en nam toe van $3 \%$ in de oudste tot $11 \%$ in de meest recente curricula. Met betrekking tot de CanMEDS-competentiegebieden stemde het merendeel van de humanistische onderdelen overeen met de competentiegebieden Professionaliteit (35\%), Medisch handelen (31\%) en Communicatie (25\%). Hoewel in de loop der tijd het aantal humanistische competenties in internationale oncologiecurricula is gestegen, blijft het algehele aandeel laag en vertegenwoordigt dit een grotendeels westers beeld van wat humanisme in de gezondheidszorg inhoudt. De vertegenwoordiging van humanisme richt zich hoofdzakelijk op de I.E.C.A.R.E.S.-kwaliteiten Respect, Medeleven en Empathie.

Hoofdstuk 5: Naarmate ons werk aan Hoofdstukken 2 tot en met 4 vorderde, waarbij onderzoeksvragen 1 tot en met 3 werden behandeld, werd duidelijk dat er behoefte bestond aan meer verdiepend en breder onderzoek naar de vraag hoe internationale curricula worden geconceptualiseerd, welke uitdagingen daarbij komen kijken en welke waargenomen factoren kunnen helpen deze uitdagingen het hoofd te bieden. In Hoofdstuk 5 problematiseren we het concept en de invoering van internationale oncologiecurricula en hun relatie tot plaatselijke machts- en cultuurcontexten. Dit deden we door middel van individuele semigestructureerde diepte-interviews. Door iteratieve 
analyses werden de betekenis en de gevolgen van de thema's besproken en verfijnd, wat uiteindelijk resulteerde in een begripsanalyse van de neokoloniale betrekkingen die door pogingen om internationale oncologiecurricula te ontwikkelen en in te voeren werden gereproduceerd en/of bestendigd. Hoofdstuk 5 ging in op onderzoeksvraag 4: "Hoe worden internationale oncologiecurricula geconceptualiseerd en ingevoerd en hoe verhouden deze curricula zich tot plaatselijke machts- en cultuurcontexten?" Door middel van deze interviews onderscheidden we vijf belangrijke uitdagingen voor internationale curricula, te weten: 1) dubbelzinnige of tegenstrijdige meningen over het doel en de omvang van internationale oncologiecurricula; 2) onvolledige vertegenwoordiging van de diverse zienswijzen en realiteiten bij het maken van de definitieve curricula; 3 ) een rigoureuze begripsvorming van de vereiste competenties; 4) een slechte afstemming tussen curriculumeisen en de plaatselijke context; en 5) de invloed van machtsrelaties en besluitvormers. Mogelijk kunnen we deze uitdagingen het hoofd bieden door juist de sterke punten van diversiteit ten volle te benutten, bijvoorbeeld door vertegenwoordiging te bevorderen, machtsverschillen aan te pakken en rekening te houden met plaatselijke contexten. We concludeerden dat internationale oncologiecurricula een belangrijke rol kunnen vervullen bij het behartigen van belangen binnen het gezondheidszorgstelsel. Het benutten van diversiteit kan een positieve invloed hebben op de gemeenschappelijke uitdagingen die bij de constructie en invoering van internationale oncologiecurricula komen kijken.

Hoofdstuk 6: In Hoofdstukken 2 tot en met 5 constateerden we een steeds terugkerend probleem, namelijk de uitdaging om internationale oncologiecurricula in te voeren. Afgezien van deze constatering waren er echter geen gegevens beschikbaar die inzicht konden bieden in meer specifieke aspecten van deze implementatie-uitdagingen. In Hoofdstuk 6 verrichten we een internationaal transversaal vragenlijstonderzoek. Het doel van deze studie was om inzichtelijk te maken welke factoren volgens de Nationale Oncologieverenigingen van invloed zijn op de invoering van het Europese Basiscurriculum voor Bestralingsoncologie/Radiotherapie (het ESTRO Core Curriculum). We constateerden dat de inhoud van het ESTRO core curriculum en de waarden die het uitdraagt door verschillende geopolitieke regio's worden onderschreven. We constateerden echter ook belemmeringen voor de invoering van het curriculum, zowel op organisatorisch niveau als op systeemniveau, zoals onvoldoende onderwijspersoneel, gebrek aan sturing en behoefte aan sterk leiderschap. Deze gegevens toonden aan dat een organisatie die bij inspanningen om haar basiscurriculum te herzien geleidelijk aan de vertegenwoordiging van geopolitieke diversiteit heeft opgevoerd, steun heeft verkregen voor de inhoud en waarden die in het curriculum worden behandeld. Als we deze conclusies door een antikoloniale lens bezien, wordt duidelijk dat we aandacht moeten blijven besteden aan het proces waarmee consensus wordt bereikt en aan de mogelijkheid dat plaatselijke diversiteit verloren gaat met alle gevolgen voor de zorg van dien. Bij het ontwikkelen 
en invoeren van internationale curricula moeten we aandacht blijven besteden aan machtsrelaties. Na dit succes in procesleiderschap waren we in staat om gebieden aan te merken die nader onderzoek en herziening behoeven, zoals een focus op training op het gebied van implementatiewetenschap en verandermanagement voor degenen die belast zijn met de invoering en aanpassing van curricula op landelijk niveau. Tevens constateerden we opnieuw dat internationale curricula een rol kunnen spelen bij het behartigen van belangen, met name op het terrein van onderwijsformatie en financiering van onderwijs en opleiding.

Hoofdstuk 7: In Hoofdstuk 7 presenteerden we een discussie waarin we samenvatten hoe Hoofdstukken 2 tot en met 6 antwoord gaven op de onderzoeksvragen en ingingen op de algemene sterke punten en beperkingen van het werk in dit proefschrift. Ten slotte wordt stilgestaan bij de vraag hoe dit werk bestaande kennis over de ontwikkeling en invoering van nationale oncologiecurricula heeft verruimd. We sluiten het proefschrift af met een bespreking van de vervolgstappen in dit vakgebied. 



\section{Valorisation}

\section{Social Relevance}

The incidence of cancer is rapidly growing globally. Consequently, the existing resources to treat cancer are inadequate and many people have little or no access to cancer care. These access issues are especially poignant in low-middle income countries. To mitigate these limitations to accessing cancer care there is a global call to double the cancer professionals by 2035. This has led to increasing efforts to develop global curricula for cancer. The overall aim of this dissertation was to explore the motivations behind and the unintended effects of creating and implementing global curricula in oncology. Using a critical approach, inspired by the work of anti-colonial scholars, we have demonstrated how power and bias influence the development and implementation of global oncology curricula. A number of conclusions and implications can be drawn from the work presented in this dissertation. First, what is considered legitimate knowledge and is therefore prioritized is heavily oriented to the Euro-American, Western perspective. There is a focus on medical expertise underpinned by the biomedical model which may be a factor in the mismatch between curricula and local healthcare needs. Next, diversity plays a critical role in the development and implementation of global curricula. Without attending to diversity there is a mismatch between what is described and what is needed or even possible in local contexts. Finally, global curricula can have a role as an advocacy tool within the healthcare system. They can be used to secure faculty time for training, training positions and other resources. We have identified that resources for implementation remain a significant challenge for global curricula and using these curricula as advocacy tools on the national and international level may be a solution.

\section{Target groups}

Our work is of potential interest to several groups. These include curriculum developers, those using the curriculum, government agents and funders.

This work will be of interest to curriculum developers because it points to current gaps in processes for development and implementation that may be limiting the application of global curricula in local contexts. These gaps include inadequate representation in the development process and an inattention to diverse competency priorities including humanism. While global standards are important in cancer care, our work shows that standardizing all curricular content may inadvertently eclipse important local imperatives and needs. 
For those who are looking to implement global curricula our work may assist them in navigating common challenges associated with diverse educational and health care systems. Specifically, our work demonstrates that change management strategies must incorporate a process for achieving alignment between local health system priorities and the global standards.

Our work will be of interest to those who have regulatory authority or funding authority over national curricular decisions. We have identified the role global curricula play as system advocacy tools including for length of training, training resources and teaching faculty.

\section{Products}

Our work has provided an archive of existing global curricula in the area of cancer care and a summary of the content of these curricula. This can be a resource to educators and health policy planners.

The recommendations from this dissertation can be directly applied to improve future efforts to develop and implement global curricula, to current efforts to implement global curricula and may be applied to graduate level courses on global health and education.

\section{Innovation}

Using a critical, anti-colonial stance, we have explored the perceived premises for global curricula and existing challenges. By using oncology as a case example, we investigated unintended aspects of pursuing global curricula that do not actively consider the historical socio-political relationships that have perpetuated differences in health professional education around the world. Positivist ontology and epistemology are dominant in the field on oncology. As we developed our work, with the hopes of speaking to oncologists and oncology educators and a desire to be published in oncology journals we had to balance these differences in ontology and epistemology between what is common in oncology and our approach in this dissertation. We hope in the future, this work will serve as a foundation to advance research that foregrounds power relations and that offers a more nuanced line of inquiry within the field of oncology. We believe our work has added to medical education literature in this capacity.

\section{Implementation}

The data from this dissertation has been presented at several national and international meetings and has been published in peer-reviewed journals. We have also shared these findings with key stakeholders influential in global curriculum design who may implement some of the recommendations in future efforts of global curriculum development in oncology. The principal author is an oncology educator who also participates in various international forums and will have ongoing opportunities to shape the development and implementation of global oncology curricula. 


\section{Acknowledgements}

My parents (Gary and Jane) - Thank you for giving me the love of learning. Your endless support and patience is the foundation which supports me.

Inesa Mary - You inspire me to make positive changes in the world. Thank you for being who you are.

Gail Giuliani- Thank you for the allowing me to use your beautiful art for my book cover page. You taught me so much about art.

Barbara-Ann Millar - You are an inspirational educator, oncologist and mother. I am so fortunate to work with you every day.

GRaCE Collaborators- Sandra Turner, Jesper Eriksen, Kim Benstead, and Lucinda Morris thank you for your inspiring work and for supporting me throughout this journey.

Heather Sampson - Thank you for encouraging me to follow my path in medicine.

Mary Gospodarowicz - You gave me a passion for global cancer control and are a true advocate for education. Thank you for being a mentor and friend.

My supervisors - Our work together has transformed my thinking and will be instrumental in shaping my career. I am so fortunate to have had the opportunity to learn from you. I look forward to our continued collaborations.

Padraig Warde - You started me on the path for a career in Radiation Oncology. Your advice and friendship is invaluable.

Pamela Catton - An inspirational mentor, taken from us too soon in 2014. Thank you for inspiring me to follow my passion for medical education. You forever changed my career for the better.

Study participants - Thank you for giving your time and knowledge to better improve our understanding of global cancer education. 



\section{About the author}

Meredith Elana Giuliani was born in Toronto, Canada on January 19, 1983. She studied at the St George's Hospital Medical School at the University of London, England from 2002 to 2007 where she received her MBBS qualifications. She completed her residency training at the University of Toronto in Radiation Oncology from 2007 to 2012. During this time she completed her Master's of Education at the University of Toronto (2010). She is currently an Associate Professor in the Department of Radiation Oncology at the University of Toronto and the Director of Education for Princess Margaret Cancer Center. In her clinical practice as a Radiation Oncologist she specializes in the treatment of head and neck and lung cancers.

Her main research interests include the influence of globalization on medical education, global cancer control and equity in healthcare. Her personal interests include travelling and exploring different cultures, art and chasing her toddler around. 



\section{SHE dissertation series}

The SHE Dissertation Series publishes dissertations of PhD candidates from the School of Health Professions Education (SHE) who defended their PhD theses at Maastricht University. The most recent ones are listed below. For more information go to: www. maastrichtuniversity.nl/she.

Kikukawa, M. (17-12-2019) The situated nature of validity: Exploring the cultural dependency of evaluating clinical teachers in Japan

Kelly, M. (10-12-2019) Body of knowledge. An interpretive inquiry into touch in medical education

Klein, D. (06.11.2019) The performance of medical record review as an instrument for measuring and improving patient safety

Bollen, J. (01.11.2019) Organ donation after euthanasia: medical, legal and ethical considerations

Wagner-Menghin, M. (25-09-2019) Self-regulated learning of history-taking: looking for predictive cues

Wilby, K. (02-07-2019) When numbers become words: Assessors' processing of performance data within OSCEs

Szulewski, A. (20-06-2019) Through the eyes of the physician: Expertise development in resuscitation medicine

McGill, D. (29-05-2019) Supervisor competence as an assessor of medical trainees; Evaluating the validity and quality of supervisor assessments

Van Rossum, T. (28-02-2019) Walking the tightrope of training and clinical service; The implementation of time variable medical training

Amalba, A. (20-12-2018) Influences of problem囚based learning combined with community凶based education and service as an integral part of the undergraduate curriculum on specialty and rural workplace choices 
Melo, B. (12-12-2018) Simulation Design Matters; Improving Obstetrics Training Outcomes

Olmos-Vega, F. (07-12-2018) Workplace Learning through Interaction: using socio-cultural theory to study residency training

Chew, K. (06-12-2018) Evaluation of a metacognitive mnemonic to mitigate cognitive errors

Sukhera, J. (29-11-2018) Bias in the Mirror. Exploring Implicit Bias in Health Professions Education

Mogre, V. (07-11-2018) Nutrition care and its education: medical students' and doctors' perspectives

Ramani, S. (31-10-2018) Swinging the pendulum from recipes to relationships: enhancing impact of feedback through transformation of institutional culture

Winslade N. (23-10-2018) Community Pharmacists' quality-of-care metrics. A prescription for improvement

Eppich, W. (10-10-2018) Learning through Talk: The Role of Discourse in Medical Education

Wenrich, M. (12-09-2018) Guided Bedside Teaching for Early Learners: Benefits and Impact for Students and Clinical Teachers

Marei, H. (07-09-2018) Application of Virtual Patients in Undergraduate Dental Education

Waterval, D. (26-04-2018) Copy but not paste, an exploration of crossborder medical curriculum partnerships

Smirnova, A. (04-04-2018) Unpacking quality in residency training and health care delivery 